\title{
Psychosocial risk factors and coronary heart disease
}

Citation for published version (APA):

Bages, N. (2000). Psychosocial risk factors and coronary heart disease. [Doctoral Thesis, Maastricht University]. Universiteit Maastricht. https://doi.org/10.26481/dis.20000323nb

Document status and date:

Published: 01/01/2000

DOI:

10.26481/dis.20000323nb

Document Version:

Publisher's PDF, also known as Version of record

\section{Please check the document version of this publication:}

- A submitted manuscript is the version of the article upon submission and before peer-review. There can be important differences between the submitted version and the official published version of record.

People interested in the research are advised to contact the author for the final version of the publication, or visit the DOI to the publisher's website.

- The final author version and the galley proof are versions of the publication after peer review.

- The final published version features the final layout of the paper including the volume, issue and page numbers.

Link to publication

\footnotetext{
General rights rights.

- You may freely distribute the URL identifying the publication in the public portal. please follow below link for the End User Agreement:

www.umlib.nl/taverne-license

Take down policy

If you believe that this document breaches copyright please contact us at:

repository@maastrichtuniversity.nl

providing details and we will investigate your claim.
}

Copyright and moral rights for the publications made accessible in the public portal are retained by the authors and/or other copyright owners and it is a condition of accessing publications that users recognise and abide by the legal requirements associated with these

- Users may download and print one copy of any publication from the public portal for the purpose of private study or research.

- You may not further distribute the material or use it for any profit-making activity or commercial gain

If the publication is distributed under the terms of Article $25 \mathrm{fa}$ of the Dutch Copyright Act, indicated by the "Taverne" license above, 


\section{PROEFSCHRIFT}

Ter verkrijging van de graad van doctor Aan de Universiteit Maastricht

Op gezag van de Rector Magnificus, Prof. Dr.A.C.Nieuwenhuijzen Kruseman Volgens het besluit van het College van Dekanen, In het openbaar te verdedigen op Donderdag 23 maaur 2000 om 12.00 uur

door

NURI BAGES

Geboren 17 september 1950 te Barcelona 
Promotor

Prof.Dr.A.P.W.M.Appels

Co-promotor

Dr.P.Falger

Beoordelingscommissie

Prof. Dr. H.W van der Borne (voorzitter)

Prof. Dr.F.W.H.M.Băr

Prof. Dr. J.J.C.B.Bremer

Prof. Dr. J.Dekker(Universiteit Utrecht)

Dr. C.M.G.Meesters

Finantial support by Universidad Simón Bolivar is gratefully acknowledged

Cover Design by Manuel Hernández. Bagés

Lay-out by Yanira Herrera and Nuri Bagés 


\section{CONTENTS}

CHAPTER 1

INTRODUCTION

1.1.BIOLOGICAL AND PSYCHOSOCIAL RISK FOR CARDIOV ASCULAR

DISEASES

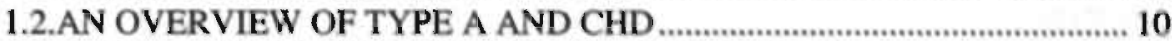

1.3.AN OVERVIEW OF VITAL EXHAUSTION AND CHD …........................ 28

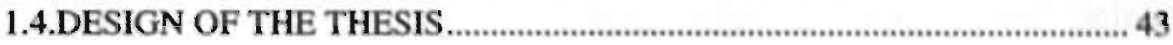

CHAPTER 2

TYPE A BEHAVIOUR PATTERN AND CARDIOVASCULAR REACTIVITY IN MANAGERS

2.1. SUMMARY

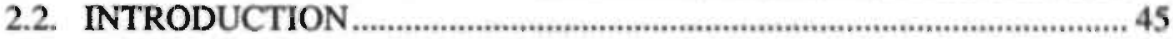

2.3. METHOD

2.4. RESULTS

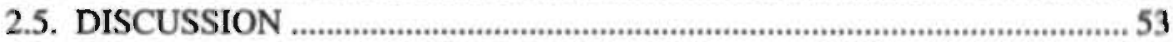

CHAPTER 3

DIFFERENCES BETWEEN INFORMANTS ABOUT TYPE A, ANGER, AND SOCLAL SUPPORT AND THEIR RELATIONSHIP WITH BLOOD PRESSURE

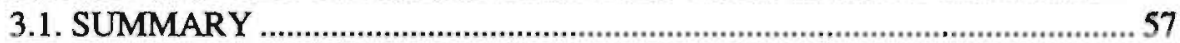

3.3. METHOD

3.4. RESULTS

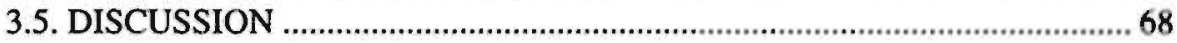

CHAPTER 4

CONSTRUCT VALIDITY OF TYPE A BEHAVIOUR PATTERN IN VENEZUELA

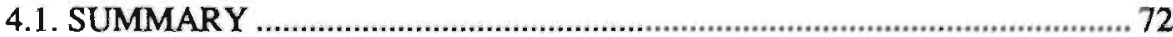

4.2. INTRODUCTION

4.3. METHOD

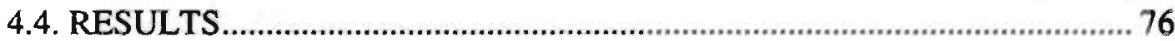

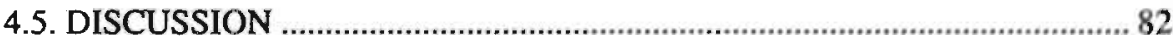

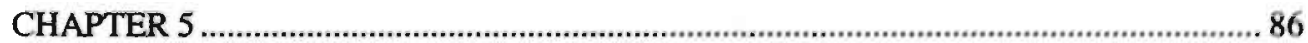

MEASURES OF VITAL EXHAUSTION AND THEIR ASSOCIATIONS WITH CORONARY HEART DISEASE RISK FACTORS IN A SAMPLE OF SPANISHSPEAKERS

5.1. SUMMARY

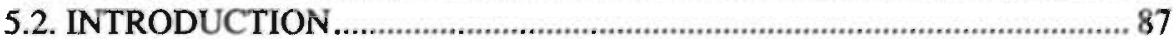

5.3. METHOD

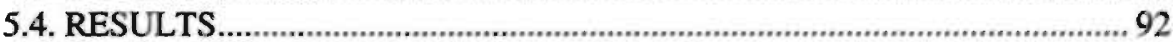

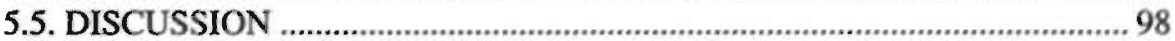


VITAL EXHAUSTION AS A RISK FACTOR OF MYOCARDIAL INFARCTION: A CASE-CONTROL STUDY IN VENEZUELA

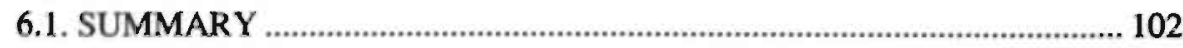

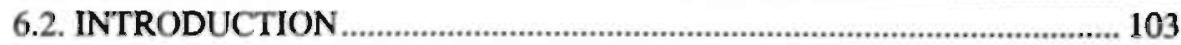

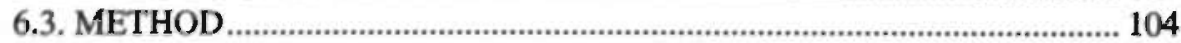

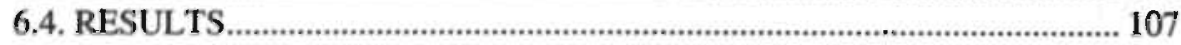

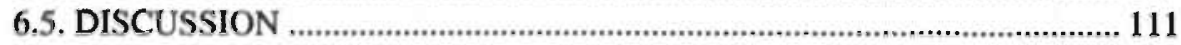

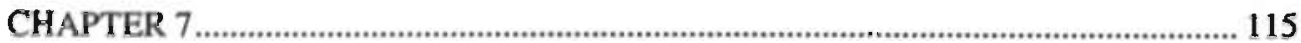

MAIN RESULTS AND GENERAL DISCUSSION

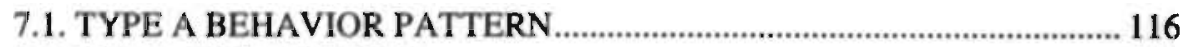

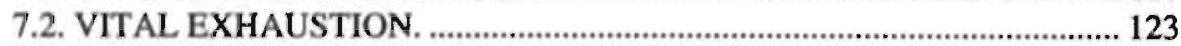

7.3. SOCIODEMOGRAPHIC ISSUES IN PSYCHOSOCIAL RISK

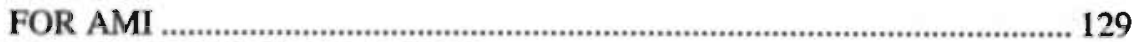

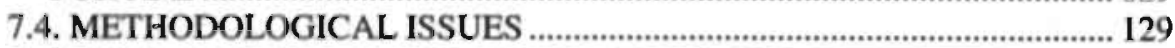

7.5. FINAL COMMENTS AND IMPLICATIONS FOR THE FUTURE .......... 133

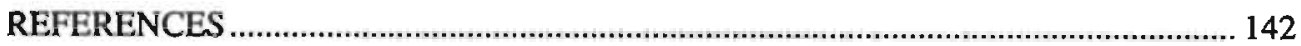

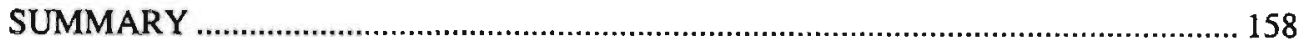

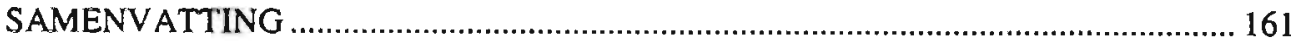

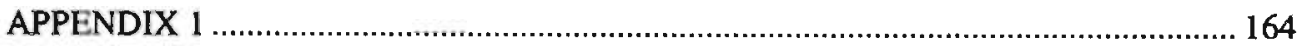

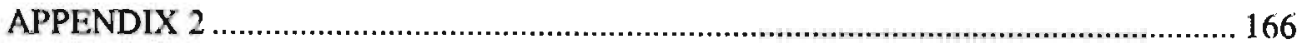

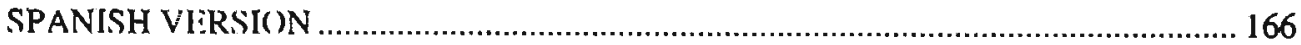

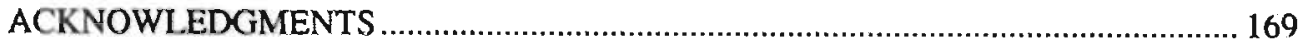

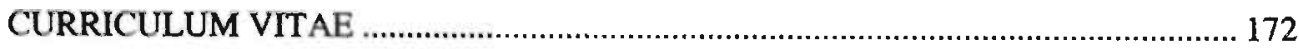




\section{CHAPTER 1}

\section{INTRODUCTION}

\subsection{BIOLOGICAL AND PSYCHOSOCIAL RISK FOR CARDIOVASCULAR DISEASES}

The complex nature of cardiovascular functioning, and malfunctioning, invites numerous questions and has done so over recent decades. Although the search for causes and determinants has resulted in important advances in its prevention, cardiac disease remains as the primary cause of morbidity and mortality in industrialised and developing countries (Bracke and Thoresen, 1996)

Why do some people develop heart problems, how do they start and how can they be prevented? Why do atheromes form, why do arteries become blocked and why do some individuals have recurrence of their heart attacks while others do not? It would be narve to think that one single line of research or unique factor will provide the "final answer" to these questions.

Health is no longer a concem exclusive to the medical profession, others outside the medical field have embarked, interdisciplinary, upon cardiac research adding important and relevant information to what is known from a biological perspective.

Proposals about the aetiology of cardiovascular disease range from the purely biological origin to a complex combination of structural, physiological, psychosocial, cultural and even geographical variables.

\subsubsection{The biological perspective}

From a biological viewpoint, cardiac risk factors include age, male sex, high blood pressure, high low-density lipoprotein (LDL), low high-density lipoprotein (HDL), lack of physical exercise, obesity, and diabetes mellitus. (Scheidt, 1996). Also, smoking and a positive family history of cardiac problems are considered major risk factors (Allan, 1996). In women, use of oral contraceptives is also considered an important risk: factor (Brezinka \& Padmos, 1994). These determinants, usually not alone but in combination, lead to atherosclerosis, a disease of the arteries which, for several reasons, results in partial or total reduction of blood flow. In this case we are particularly concerned with the coronary arteries that irrigate the heart muscle. In general, this is referred to as coronary artery (or heart) disease (CAD or 
CHD). In the atherosclerotic process there is a narrowing of the arterial lumen due to the formation of plaques mostly composed of fibrous tissue (about $80 \%$ ), extracellular lipids and calcium (about 5\%) (Roberts, 1996). The results of atherosclerosis may go unnoticed by the person, i.e., they can be asymptomatic (silent ischemia) or can result in severe chest pain (angina), and myocardial infarction (MI). In all cases there is a failure in the flow of blood to specific portions of the heart muscle. In a study carried out by Roberts (1996) it was found that in 80 patients who died of MI, Sudden Cardiac Death (SCD) or Unstable Angina Pectoris (UAP), autopsy analysis showed that they had an average of 2.9 of the major coronary arteries severely narrowed at some point.

The biological risk factors may combine to increase the risk but the hypothesis that they interact having a potentiating effect is widely accepted (Evans, 1995)

Although all the above-mentioned biological risk factors have shown to be strong predictors for CHD, there are controversial results in the literature about the strength of their predictive nature. Some authors have concluded that although there is an impressive number of studies where the biological risk factors prove their predictive value, there is still a large proportion of CHD which seems to occur for other reasons (Patel, 1983; Dembroski \& MacDougall, 1983).

\subsubsection{Why talk about psychosocial risk factors?}

The role of the traditional risk factors for CHD, such as serum cholesterol, blood pressure and smoking, started to be questioned in the mid 1950s as they did not appear to fully explain neither the rapid rise of disease in the 20th century nor its geographical distribution (Rosenman, 1991a; Jenkins, 1988). By that time it became evident that the problem was associated with urbanisation and industrialisation but little attention was paid to the psychosocial and economical variables involved in the process (Rosenman \& Friedman, 1959).

The issue of how psychosocial factors may influence the atherosclerotic process is still unresolved. Roberts (1996) argues that psychosocial factors play a major role not in producing the atherosclerotic plaque but in determining when a major cardiac event will occur. This view is somewhat limited as most of the so-called biological risk factors have a behavioural component. It is mostly a person's own choice, and therefore psychosocial (cognitive, affective or behavioral), to prefer a healthy or unhealthy diet, to smoke, or to exercise or not. Many of the biological risk factors have a behavioural origin and it has even been proposed that CHD is fundamentally a behaviourally moderated disease (Jenkins, 1988). 
What seems to be of great importance is the multi-factorial nature of risk in the aetiology of CHD. The study of interactions between biological and psychosocial risk indicators is currently one of the most profitable and promising areas in behavioral medicine. The combined role of psychosocial, and biological factors in myocardial ischemia is clearly demonstrated in a recent study by Torosian, Lumley, Pickard \& Kettere, (1997). They examined the relationship of psychological, cardiac, and general medical history factors to asymptomatic versus symptomatic myocardial ischemia in 102 patients who underwent treadmill exercise and showed perfusion imaging indicative of ischemia. Their findings showed that patients with silent ischemia had higher anger control and externally oriented thinking but did not differ on depression or global alexythimia from symptomatic patients. These results remained after controlling for cardiac factors, thus strongly suggesting that psychological variables play a major role in cardiac disease.

Although it is undeniable that CHD mortality rates have declined in the past years due to new therapeutic modalities (e.g. PTCA, angiography, CABG) (Sprafka, Folsom, Burke, Hahn, \& Pirie, 1990), the reduction of risk through changes in health habits (diet, exercise and smoking) and psychological characteristics (stress, Type A, anger, social support) is also irrefutable (Scherwitz \& Ornish, 1990)

In some countries of the world changes in therapeutic modalities have taken place and an increasing number of prevention and rehabilitation programs are comprehensive and include pharmacological and psychosocial interventions (Allan \& Scheidt, 1996). The work of Scherwitz. \& Ornish and their collaborators in cardiac rehabilitation is an excellent example of the possibilities of combined interventions. The striking results of research in the psychosocial domain regarding heart problems have largely contributed to this increase in credibility and, thus, in the application of all-inclusive programs.

There are still several issues to be resolved within this interactional framework. Controversial results regarding universality of the psychosocial constructs gender differences, associations of psychosocial factors, among themselves and with biological indicators, sociodemographic influences in cardiac risk, and several others deserve further attention. It is the purpose of this thesis to focus on some of those problems from this interactional point of view.

Before going into detail about the main aspects covered in this dissertation it is important to review the underlying principles of Behavioral Medicine and the emerging field of Cardiac Psychology as they constitute the theoretical support of most of the topics presented hereafter. 


\subsubsection{Behavioural Medicine. Brief history and present perspectives}

Weiss (1992) places the birth of Behavioural Medicine in 1977 during the Yale Conference on Behavioral Medicine but acknowledges its origins in the early philosophers, Homer, Plato and Aristotle and their propositions about mind-body relationships.

In the last two decades the field of Behavioural Medicine has made significant contributions in the areas of health promotion, disease prevention, curative and rehabilitation processes. During this time Behavioral Medicine has brought about a wider and better understanding of aspects related to well-being and disease and has helped to influence health politics in many countries of the world.

As a result of theoretical and empirical findings the importance of psychosocial aspects, such as behaviours, cognitions, and affects, as well as sociological and environmental factors on health and illness has been widely recognized and research into health and illness now commonly embraces both, physiological and psychological perspectives.

Moreover, this broader multidisciplinary approach goes against single-point-of-view interpretations in the treatment of health problems. As Weiss (1983) had already pointed out, "...Thus, the genetic, nutritional, constitutional, biochemical, physiological, behavioural, and sociocultural variables must be recognized for their relative contributions to the complete picture of the (health) condition in question" ( $\mathrm{p} 9$ ).

Weiss proposed a broader basis of Behavioral Medicine which included 1) the intrapersonal, interpersonal and environmental levels of analysis, 2) the areas of application individuaVfamilial, health services, school/work and community, and 3) processes related to health behaviours, changes in behaviours related to illnesses, and maintenance of healthy behaviours. Thus a tri-dimensional model emerged which is essential in today's perspective of Behavioral Medicine. Based on this model, the onset, development, and treatment of illness are better understood when all the levels of analysis are included. Each of them (society, family, individual, organism, tissues, cells, etc.) may mutually influence and each level has its own distinctive qualities and organisation deserving different methods of study and interpretation. The application of the tri-dimensional model provides an overview of the diverse situations that may appear in relation to health and illness from which the most appropriate level of intervention can be selected.

According to the particular situation of the individual at a given moment, one of the levels may be predominant. This could be the social or familial level or even the cultural one. The effects or consequences of an illness may be better understood if individual resources, 
physical or psychological, are evaluated or if the way in which the surrounding environment co-operates is understood.

Based on all the previous notions, the International Society of Behavioural Medicine defines it as an "interdisciplinary field concerned with the development and integration of behavioural and bio-medical science knowledge and techniques relevant to health and illness and the application of these techniques to prevention, diagnosis, treatment and rehabilitation" (Stress Research Reports, 1994). This definition highlights the necessity for interdisciplinary contributions in order to cover all the levels involved in the tri-dimensional model. The combination of biomedical, behavioral and psychosocial variables in seeking a better understanding of the processes of health and illness, is essential. This requires co-operation of knowledge and technology from many disciplines (Orth-Gomer \& Weiss, 1994).

Agras (1982) had already pointed to the need to define Behavioral Medicine as a communication network across a wide spectrum of disciplines that had not been previously connected. From the medical point of view, poses Agras, the interest in knowing how the environmental and behavioural factors interact with physiological and biochemical processes in the development, course and treatment of illness is revived.

This communication network should aid the implementation of techniques and intervention programs to induce changes in lifestyle, which perhaps in combination with pharmacological therapies will contribute to primary and secondary prevention in cardiac diseases.

Thus, Behavioural Medicine points to the integration of disciplines and/or levels of analysis in both research and application areas. Concerning specifically cardiovascular problems, it represents the supporting background or model of study leading researchers or clinicians to incorporate multivariable analysis and integrative interpretations into their investigations or clinical practice.

Recently in the United States the field of Cardiac Psychology has emerged as a result of the interdisciplinary approach proposed by Behavioral Medicine (Allan \& Scheidt, 1996). It is a new speciality that includes elements from the biological and psychosocial domains. There is no formal definition of Cardiac Psychology, as the term is self-defined. It is concerned with all aspects of cardiac health and illness where psychological variables may intervene. As Allan (1996) points out, Cardiac Psychology is the discipline that mostly contributes to the bulge of information in behavioral medicine. Both, Behavioral Medicine and Cardiac Psychology have a particular interest in the assessment and prevention of the risk that an individual, a group, a community or a society may have for the onset, development or 
maintenance of a cardiac problem. Rehabilitation and psychopharmacology are also part of their main concerns.

In this introduction chapter the main focus of attention is on Type A Behavior Pattern (TABP) and Vital Exhaustion (VE), two specific psychosocial risk factors for CHD. The existing evidence concerning their role in the pathogenesis of CHD will be briefly presented. Other risk factors such as anger expression lack of social support, and cardiovascular reactivity, together with sociodemographic aspects such as gender and culture, will be delineated in as much as they are linked to TABP and VE.

\subsection{AN OVERVIEW OF TYPE A AND CHD}

\subsubsection{Origins and Definitions}

As a result of the failure of the traditional risk factors to explain more than about half of the prevalence and incidence of CHD in the USA and on the basis of their clinical observations, Friedman \& Rosenman (1959) described a pattern of behaviour which seemed to be characteristic of their CHD patients and which was exhibited far less in persons without CHD. In their first study carried out in a group of tax accountants, it was found that when participants reported time pressure due to approaching deadlines, a significant rise of serum cholesterol and acceleration of blood clotting was observed. These results stimulated research in individuals with a chronic sense of time urgency. It was then observed that time urgency was accompanied by other behaviours. At this stage, a complex pattern of behaviour characteristic of CHD patients was fully described and termed Type A or Type B Behaviour Paitern (TABP) (Rosenman \& Chesney, 1982).

Friedman and Rosenman were not the first to suggest that specific behavioural characteristics seemed to be present in heart patients (Chesney, Eagleston \& Rosenman 1981; Patel, 1983), but they presented their information in a systematic manner, gave it a name and developed an evaluation method to examine its value as a risk factor for CHD in a major epidemiological study, the Western Collaborative Group Study (WCGS) (Rosenman, Brand, Jenkins, Friedman, Straus \& Wurm, 1975; Rosenman, Brand, Scholtz, \& Friedman,1976)

Friedman and Rosenman (1959) described the Type A person as displaying an overt behaviour pattern characterized by 'intense ambition, competitive 'drive', constant preoccupations with occupational 'deadlines' and a sense of time urgency". More recently, 
Rosenman (1990) gave a more "sophisticated" definition of Type A as "an action-emotion complex involving behavioural dispositions such as ambitiousness, aggressiveness, competitiveness and impatience; specific behaviours such as muscle tenseness, alertness, rapid and emphatic vocal stylistics and accelerated pace of activities, and emotional responses such as irritation, hostility and an increased potential for anger"

Other authors have defined TABP in their own words and although they may convey the same meaning, it is interesting to note the different ways of expressing it. Evans (1995), for instance described Type As as having a competitive craving for achievement and recognition, a tendency towards hostility and aggression and a sense of extreme time urgency and impatience. He emphasises that Type As speak and act fast. Valdes and Flores (1987), on the other hand, divide Type A behaviour into " formal components" (loud voice, rapid speech, psychomotor activity), attitudes and emotions (hostility, impatience, anger), motivational aspects (need for achievement, competitiveness) and overt behaviours (speed, hyperactivity, job involvement). Jenkins, Zyzanski, \& Rosenman (1978) propose that "Type A behaviour is an overt style of reactions characterised by some or all of the following: intense striving for achievement, competitiveness, easily-provoked impatience, time-urgency, abruptness of gesture and speech, hyper-alert posture, tense facial musculature, overcommitment to vocation or profession and excesses of drive and hostility".

Opposed to Type As, Type B persons lack all or most of those characteristics of Type As, they exhibit a different coping style with no competitiveness, time urgency or hostility (Rosenman, 1990). Very little has been said about Type Bs apart from stating that Type A characteristics are not present in them (Kaplan, 1991).

The emphasis on overt behavioural manifestations rather than on emotion or psychological states is evident in all the various definitions. Moreover, psychomotor signs of Type A characteristics (facial tension, tick-like elevation of eyebrow, tense posture as indicators of impatience or hostility) rather than verbal subjective responses are the crucial aspects in the presence/absence decision about Type A characteristics (Friedman, Fleishman \& Price, 1996).

It has been strongly emphasised that TABP is not to be equated with stress situations or responses, anxiety, neurosis, worry or fear (Rosenman, 1990; Jenkins, Zyzanski \& Rosenman, 1978).

\subsubsection{The theory}

The theoretical basis of the Type A concept and its components has received relatively little attention. This is fundamental in order to understand how Type A 
appears in a person or a society, what maintains it (Glass, 1991) and under which circumstances. The scarcity of this kind of information may be an important contributor to the lack of consistency in the findings from the multiple studies on Type A and CHD. It is clear that the relationship between psychosocial factors and health is a very complex one and the poor conceptualisation of the psychosocial factors may add to the difficulty in understanding the relationship. There is a need to improve the scientific validity of the theory (Friedman, 1992).

Various researchers have made important contributions to the theoretical foundations of Type A and hostility. Very briefly, Bienias \& Strube (1991) propose a self-schema concept associated to Type A. Phillips, Freedman, Ivancevich \& Matteson, (1991) on the other hand propose that Type A is related to goal-setting and uncertainty. Van Egeren (1991) presents his "success trap" theory related to the rewarding and punishing consequences of Type A behaviour within a culture and specific moments in history. As a final example of a theoretical proposition, Fontana, Rosenber, Burg, Kerns \& Kolonese, 1991) suggest that there is an interactive risk effect between Type $A$ and self-referencing (use of self-referring in verbal or written communications, 1, me, mine, myself). Unfortunately, the empirical studies about the relationship of Type A with CHD make little use of those conceptual frameworks, an exception being a study conducted by Scherwitz, McKelvain, Laman, Patterson, Dutton, Yusim, Lester, Draft, Rochelle \& Leachman (1983) where they found that the more use of self-referencing the major the extent of the coronary atherosclerosis.

In his exposition of Type A, Van Egeren (1991) emphasises the social and economical demand differences in three periods of time, medieval, modern and post-modern. He argues that whereas Type A behaviour in the modern period was manifested in order to attain more "natural" and social goals (noble causes, specific missions, conquests), in the postmodern era the changes in the economy introduced the need to attain more materialistic goals and approval from others under a confused value system. The indicators of success are baftling and determined by others. The self-esteem of Type As is placed in jeopardy, as it has to cope with contradictions between the challenges and the reinforcement system. As Van Egeren precisely expresses it in his "trap theory" which is opposed to the proposition of Rosenman \& Chesney (1982) who state that there is a higher incidence of Type A due to the pressures of increased industrialization: "Type A behaviour has become more maladaptive (entrapped, self-defeating, counterproductive), rather than more common, because of a major shift in the socio-economic reward system of this century. In this view, we do not live in an "Age of Type A Behaviour" which, if anything, was the Victorian Age, but in an "Age of Type B Behaviour" requiring greater taming of extreme aggression and rugged individualism. It is 
precisely the poor fit of Type A behaviour to the current reward system that makes it behaviourally maladaptive and physiologically damaging".

These theoretical positions clearly emphasise the possible individual-environmentsociety interactions involved in the Type A concept and indicate the "dependence" of Type A behavior (individual level) on socially and historically established norms and rules. Thus, when investigating on psychosocial risk factors such as Type A it seems relevant to refer always to the social context of the individuals where the data is collected.

\subsubsection{Type $A$ behavior and $C H D$ : The evidence}

The most substantial evidence relating TABP to CHD came from the WCGS with follow-ups of 8.5, 22 and 27 years. (Rosenman, Brand, Scholtz, \& Friedman 1976; Ragland \& Brand, 1988a; Carmelli, Dame, Swan, \& Rosenman, 1991). At intake 3.524 white men, aged 39 to 59 , working in 10 companies were evaluated with respect to the occurrence of acute CHD death, other CHD death, nonfatal symptomatic myocardial infarction, silent myocardial infarction, and angina pectoris. Results showed that systolic blood pressure was the only consistent risk factor for all CHD manifestations and serum cholesterol was a predictor for all fatal CHD events and non-fatal myocardial infarction. Smoking was an independent risk factor for fatal $\mathrm{CHD}$ and marginally predictive for non-fatal infarction. TABP was a significant independent risk factor for acute CHD morbidity. None of the initial measures were significant risk factors for angina or silent infarction. It was concluded that TABP is significantly predictive of a "hard" CHD end-point as it is the first myocardial infarction. Type A not predicting cardiac death was attributed to the fact that post infarction mortality is generally related to the clinical consequences of the first event. On the other hand, not being predictive of other CHD problems could well mean that TABP is a risk factor for infarction but unrelated to the gradual deterioration of the heart blood vessels (Langeluddecke, Fulcher, Jones, Tennant, 1988).

Recently, a review of ten studies about Type A behavior after myocardial infarction (Cohen, Ardjoen \& Sewpersad, 1997) found that Type A, assessed either before or just after MI did not predict death or recurrent cardiac morbidity. This may have been due to the fact that Type A behavior shows a decline in heart patients after their cardiac event both in treated and non-treated groups but significantly more in the treated groups (Friedman, Fleishman \& Price, 1996; Burrell, 1996; Sebregts, Falger, Bar, \& Appels, 1999). Cohen, Ardjoen \& Sewpersas also reported that those showing reductions of Type A after their MI suffer fewer cardiac recurrences. Unfortunately, six out of the ten studies reviewed used methods different 
to the Structured Interview (SI) or Videotaped Clinical Examination (VCE) to assess Type A. (these results will be discussed in a later section about Type $\mathrm{A}$ and assessment issues).

Other prospective studies have also found evidence for the relationship between Type $\mathrm{A}$ and various $\mathrm{CHD}$ end points. The Framingham Heart study (Haynes, Feinleib, \& Kannel, 1980) and the French-Belgian Collaborative Study (Kortnitzer, De Backer, Dramaix, et al., 1982) found that Type A predicted total CHD and Myocardial Infarction (MD). Other studies have found negative results. In the Honolulu Heart Study (Cohen and Reed, 1985) in which 2200 men of Japanese descent participated, no relation between Type $A$ and incidence of total CHD, MI and angina was observed. The low incidence of CHD in this population together with the low prevalence of TABP anong Japanese men seems to account for their findings (Haynes \& Matthews, 1988) These results indicate the importance of taking into account geographical and other environmental variables when interpreting Type A results.

A 22-year follow-up analysis of the WCGS by Ragland \& Brand (1988) found that those men diagnosed as Type As in 1960 who later developed CHD actually had lower mortality rates over 22 years of observation in comparison to those diagnosed as Type Bs. Type A, the authors argue, could even be considered as a protective factor. Assessment issues may account for these findings (see later section).

Two meta-analytic reviews conducted by Booth-Kewley and Friedman, (1987) and Matthews (1988) concluded, at that time, that Type A behavior appears to be a risk factor for CHD but only when the Structured Interview is used as a diagnostic tool rather than selfadministered questionnaires (e.g. Jenkins Activity Survey). This association was strongest in çross-sectional studies.

All the investigations described up to this point were conducted in participants who were healthy at entry. There also exist a number of prospective studies that have assessed the relationship between TABP and CHD in high-risk persons, that is after a first coronary event (MI. CABG surgery) or with high risk factors (high serum cholesterol, smoking, hypertension). The Aspirin Myocardial Infarction Study (Shekelle, Gale, \& Norusis, 1985), the Multiple Risk Factor Intervention Trial- MRFIT (Shekelle, Hulley, Neaton, Billings, Borhani, Gerace, Jacobs, Lasser, Mittlemark \& Stamler, 1985) all failed to support the hypothesis that Type $\mathrm{A}$ is related to CHD after a first coronary event. The use of inappropriate assessment techniques to evaluate Type $\mathrm{A}$ is one of the possible reasons for these results, (Mathews \& Haynes, 1986) together with the fact that the illness and treatments may have had an enormous impact on the life of the patients and on their risk behaviours which most probably may have interacted with TABP; after their coronary event changes in behaviour may have resulted in a protective effect. In most of the above 
studies behavioural changes resulting from the disease are not taken into account (Bennett \& Carroll, 1990). The moment of assessment (sometimes shortly after MI) and the appropriateness of the measurement techniques for these specific populations most probably affected these results (Haynes \& Matthews, 1988; Rosenman, 1990).

A number of studies using coronary angiography patients, who had not yet suffered a coronary event in order to explore the relationship between Type A and the extent of CHD, have also found negative results (Williams, Haney, Lee, Kong, Blumenthal, \& Schanberg 1980; Blumenthal, Barefoot, Burg, \& Williams, 1987). These investigations with angiographic patients have also received methodological criticisms similar to those mentioned in the studies of high-risk individuals.

\subsubsection{The controversial findings. TABP: Global risk or toxic elements.}

After 40 years since its appearance the Type A concept is still alive although it has undergone some criticism due to controversial results that questioned its validity as a global risk factor for CHD (Dembroski \& Costa, 1978; Williams \& Barefoot, 1988) and its evaluation procedures (Essence 1991). At present, the interest in Type A as a risk factor is markedly diminished and this is reflected in a much smaller number of published journal reports compared to the late eighties and early nineties, due to a drift from global Type A research to the study of the roles of its separate components, especially hostility and anger.

All this has resulted from the negative findings in several studies about the relationship between global TABP and CHD together with the positive results in studies evaluating the relationship between anger and hostility and CHD. The negative findings in the association of TABP and CHD have been repeatedly attributed to incorrect assessment procedures which do not allow for the evaluation of fundamental manifestations of Type $A$ such as psychomotor signs, time-urgency and outward expression of anger and hostility relying mainly on self-reports (Friedman, Fleishmanin \& Price, 1996). One of the basic arguments in favour of the Type A-CHD association is that the assessment method is crucial to explore the relationship because: of Type As being unaware of some of their typical responses and therefore their inability to acknowledge them in self-report instruments. Most of the studies where no association between Type A and fatal and non-fatal MI was found relied on questionnaires and scales to establish presence or absence of TABP. Friedman, Fleishmann \& Price (1996) further add that Type As may lack awareness of or are reluctant to admit behaviours such as their impatience on easily aroused hostility and will not report them when asked through a questionnaire. These individuals will be categorised as Type Bs unless 
an observation of those behaviours is carried out through audio taped or videotaped interviews. Thus, the use of interviews when evaluating TABP seems fundamental.

On the other hand, it has been argued that assessing people as Type A or not and trying to predict cardiac problems from that classification is an oversimplification (Thoresen \& Powell, 1992). It seems necessary to focus on specific elements and /or their combined effects in order to make more accurate predictions.

The idea that some of the elements that constitute TABP may have a more powerful negative effect on cardiovascular health or that the presence of only one of them is necessary to account for the risk has appealed to many researchers (Matthews, Glass, Rosenman, \& Bortner, 1977; Dembroski \& Costa, 1987; Chesney, Hecker \& Black, 1988; Dembroski, MacDougall, Costa, Granditis, 1989; Suárez, \& Williams, 1990; Mendes de Leon \& Meesters, 1991). This belief stems mainly from the contradictory findings of large-scale studies evaluating global TABP and its relation to different CHD endpoints. In this sense it has been pointed out that assessment of global TABP is not a profitable strategy and that research should be devoted to consider separately the components of global TABP (Dembroski \& Costa, 1987). The apparent overrating of people as Type A backs this argument. It is argued that there seems to be a trend of studies in reporting no less than 70 $90 \%$ of individuals to have been evaluated as Type As, e.g. Dembroski \& MacDougall (1983), who found in two studies that $85 \%$ of top level executives and $90 \%$ of senior-level officers in the military, were described as Type A through the SI.

This high risk, argues Dembroski, is inconsistent with epidemiological results. The high prevalence of Type $A$ in these groups may simply indicate that it is this type of person that is usually considered suitable to escalate to top jobs and these findings simply confirm the idea that society reinforces Type A individuals considering them the most able to take the highest responsibilities. Hence, the high number of them at that level. As described earlier, the main components of TABP are competitiveness, time-urgency, hard-driving, anger or potential for hostility, and psychomotor behavioral manifestations such as, tense facial musculature, emphatic speech and alertness. These characteristics are present to different degrees in each individual categorised as Type A and may even be observed in the same person depending on the surrounding situation and presence of eliciting stimuli (Thoresen \& Powell, 1992). Recently, there has been a growing interest in the study of anger and hostility due to numerous reports about its specific contributions to elevated cardiac risk. Several studies have found that anger and/or hostility seem to be the "toxic" elements (Williams, Barefoot \& Shekelle, 1985; Koskenvuo, Kaprio, Rose, Kesanieme, Heikkila \& Langinvainio, 1988; Dembroski, et al., 1989; Smith, 1992) However, the evidence is controversial 
(Rosennan, 1991c; Heam, Murray \& Luepker, 1989;) and studies show different power with respect to onset and progress of CHD (Booth-Kewely \& Friedman, 1987; Helmer, Ragland \& Syme, 1991, Matthews, 1988). As with global Type A, these discrepancies are generally attributed to differences in assessment methods (Smith \& Pope, 1990, Suarez \& Williams, 1990). Moreover, the literature encompasses important studies where conceptual confusion exists as to what is meant by "anger" and "hostility". The intrinsic complexities of human emotions in general, and of anger and hostility in particular, constitute many an obstacle in research on negative emotions and illness (Averill, 1982). It goes beyond the scope of this dissertation to further discuss this conceptual issue.

\subsubsection{Type A behavior and Anger}

The substantial number of reports about the influence of "negative affect" (hostility, anger, depression) on cardiac problems makes it compelling to include it when studying any other (psychosocial) risk factor for CHD. There is considerable evidence suggesting that negative emotions are at the root of several health problems and premature death (Friedman, Tucker \& Reisen, 1995). Even recalling experiences that elicited anger in the past six months may have effects on heart physiology, specifically left ventricular function (Ironson, Taylor, Boltwood, Bartzokis, Dennis, Chesney, Spitzer, \& Segall, 1992) and coronary vasoconstriction (Boltwood, Taylorm, Burke, Grogin, \& Giacomini, 1993)

Although there is a large number of studies in which the so called "hostility complex" (Williams, 1987) has been found to be related to CHD, conceptual discrepancies and assessment variations have led to some important controversial reports. The "hostility complex" is composed of a series of conflicting emotions (e.g. mistrust, cynicism, pessimistic orientation, suspicion, resentment, irritability, and argumentativeness). All these refer mainly to cognitive attitudes and beliefs in contrast to the "anger" construct that includes more affective and, particularly, behavioural components related to the manner in which individuals express their angry feelings. There have been numerous attempts to define "hostility" and "anger" but currently the conclusion should be that the complexity of these negative feelings does not permit a single, or simple, definition.

In the studies we are undertaking here there is a particular interest. in what people actually do when feeling angry (or hostile) and in the relationship of types of anger manifestations or reactions upon (cardiovascular) health. Thus, we will refer only to studies where manifestations of anger expression, and hostility, are assessed. Also, we will refer to anger-expression styles as opposed to short-lived episodes of anger (orr acute angry) reactions. 
Historically, the term anger was already defined by Aristotle in 350 B. C. as "an impulse accompanied by pain, to a conspicuous revenge for a conspicuous slight directed without justification toward what concerns oneself or what concerns one's firiends" (Kempt \& Strongman, 1995). Moreover, he already distinguished the hot-tempered, whose anger was quick to arise but also quick to subside from the sullen. Other ancient philosophers, too, contributed to the subject, notably Seneca who, in 45 A.D. regarded anger as the desire to exact punishment. Also, he postulated that individuals differ with respect to expressing and controlling their anger (Kempt \& Strongman, 1995).

It has also been postulated that the words "anger" and "angina" have their origin in the same root word, although this seems to be coincidental (Allan \& Scheidt, 1996). Presently, according to Kempt \& Strongman (1995), there has been little change as to the definition of the concept of anger and even its assessment that in ancient times was probably based on folk psychology, and nowadays has been replaced by a few questionnaires.

Spielberger et al,, (1985) proposed a conceptual frame and assessment procedure that have been very influentiall in the field of psychosocial risk factors and coronary heart disease. In the mid-eighties this research team conceptualized "anger" by making a distinction between "Trait" and "State" anger. They defined "State" anger as "an emotional state or condition that consists of subjective feelings of irritation, annoyance, fury and rage, with concomitant activation or arousal of the autonomic nervous system", while "Trait" anger tapped on individual differences in the frequency with which the State anger would be experienced over time. (The first A of the AHA syndrome refers to: Anger, Hostility, and Aggression). The State-Trait Anger Scale became the instrument to assess anger conceptualized in that manner.

Following the early work of Funkenstein, King \& Drolette (1954), Spielberger proposes that it is important to distinguish between, and separately assess, anger expression styles (State anger), i.e. anger-out (manifestations of aggressive behaviour toward others, either persons or objects, either directly or indirectly), anger-in (directing anger towards one self by repressing or denying angry feelings), with anger-control somewhere in the middle (cool down, control temper). This is similar to the anger-in, anger-out and anger-discuss classifications derived from the Framingham Heart Study (Haynes, Feinleib \& Kannel, 1980).

Whether overtly expressing angry feelings, or withholding and exerting control over them has eicher negative or positive consequences for cardiovascular health continues to be a matter of debate at present. There seems to be ample evidence to support either point of view. It has been found, for instance, that hostility and anger both increase the risk of CHD (Dembroski \& Costa, 1987). Spielberger (1992), reviews a series of studies where 
suppression of anger was found to be associated with elevated blood pressure. Siegman, Dembroski \& Crump (1992) present evidence from correlational and experimental studies where anger expression, i.e., overt vocal signs and gestures, is a significant risk factor for CHD, whereas suppression of the experience of anger, or even the experience itself, does not have predictive power. Mendes de Leon \& Meesters (1991) also found that outward expression of anger was rnore common in cardiac patients. By contrast, anger-suppression has been found to correlate with progression of carotid atherosclerosis Julkunen, Salonen, Kaplan, Chesney \& Salonen, 1994) while other studies did not show associations of anger (or hostility) with cardiovascular responsivity (Smith \& Houston, 1987) or with coronary atherosclerosis (Tennant, Langeluddecke, Fulcher \& Wilby, 1987)

The way people feel and react when angry, in the presence of an internal or external eliciting stimulus is, implicitly, strongly related to the Type A construct. Nevertheless, it is difficult to discuss previous research results as usually hostility, rather than anger, is the construct most frequently mentioned in relation to Type A. Anger has been considered as part of the Type A pathology since its first appearance and has been related to hypertension, stroke and infarction. It has also been regarded, still with some terminological confusion, as one of the components of the so called AHA (Anger/Hostility/Aggression) syndrome arguing that it may or may not be part of the Type A construct (Friedman, 1989). Anger has also been related to time-pathologies (Ulmer \& Schwartzburd, 1996) and thus, indirectly to Type A as impatience and time pressure is one of its defining characteristics. It seerns most relevant to continue investigating this field specially by focusing on the associations of anger-expression styles with Type A behavior.

\subsubsection{Assessment issues}

The topic of assessment is crucial both within Type A and anger-expression research. It is now widely accepted that interviews (Structured Interview, (SI) (Rosenman, 1978) and Videotaped Clinical Examination, VCE (Friedman \& Powel, 1984; Friedman, Fleishmannn \& Price, 1996) rather than questionnaires should always be preferred as the main evaluation procedure given that what is of crucial importance is not what people saty but how they say it (Williams, 1984).

Most of those studies in which global Type A was not found to be a predictor of cardiac events relied on questionnaires to assess Type A. It may be valid to use self-reports within a study on Type A but only as a secondary source of information and, perhaps, for comparison purposes between self-report and interview data. 
According to Friedman, Fleishmann \& Price (1996), the existing confusion about the predictive value of Type A behaviour stems mainly from the fact that as with any other medical disorder, Type A cannot be self-diagnosed. In making particular reference to hostility Friedman ett al. argue that people are unaware of their hostile reactions and those who are, will be careful not to acknowledge it when asked. The same may apply to other Type A components, such as time-urgency, which although not always socially undesirable, may have become "normal" for the Type A individual and will, therefore, not be reported as notorious.

Questionnaires, and even behavioural evaluations such as the SI, normally ask general questions about attitudes and/or behaviours that are unrelated to situations, circumstances, time frames or relevant others. In this particular areat of research where specificity seem to be particularly relevant when trying to pin down what a risk factor is and what is not, the inclusion of the social context and other environmental aspects seems essential. In fact, the social context has been reported as being particularly important at least in the study of hostility as it may provide a more complete understanding of the biopsychosocial processes linking hostility and health (Smith \& Christensen, 1992). Type A and anger may also be included in this line of thinking implying the need for assessment under different social circumstances. In fact, this may contribute to the testing of the possible links between those psychosocial characteristics and cardiovascular disease indicators.

With specific reference to anger Chesney (1985) strongly suggests the assessment by observing the individual in the natural environment, although the difficulties involved namely, the influence of the observer and the costs in terms of personnel, decoding and analysis of data, are acknowledged. Thus, whenever it is possible to assess Type A and anger by means of interviews or direct observations this would be the most advisable method, and the one that should bring about the more reliable data. When questionnaires are the only possible method however, then asking observers close to the participants to assess their behavior may be a valid method to contrast with self-perception.

\subsubsection{The hyperreactivity hypothesis}

How do emotions and behaviour lead to heart damage? Of all the hypotheses postulated so far in the literature the hyperreactivity one, based on chronically increased activity of the sympathetic nervous system, is the most widely used. Ennotions, either negative or positive, may have an adverse impact on the heart (Powell, 1996). This issue may be examined from two different perspectives. First, hyperreactivity of the cardiovascular response to external stimuli leads to the progressive deterioration of the cardiovascular system 
due to frequent, and also intense, cardiovascular reactivity when inadequately coping with daily challenges (Type A, anger). Second, cardiovascular malfunctioning may be triggered by acute emotional reactions to specific stressors. Probably a combination of the two would be the most plausible proposition. In fact, there is strong evidence to suggest that abrupt reactions to daily hassles may be the most important trigger of cardiac events (Powell, 1996; Rozanski, Blumenthal \& Kaplan, 1999). It seems logical to assume that when this happens a previous history of continuous over-reactivity over prolonged periods of time may have been present. Powell (1996) strongly emphasizes the role of beliefs and attitudes, obviously shaped over the life span, as key aspects of the emotional over-reactivity response.

A full account of all the hypotheses about the link between psychosocial risk factors and cardiac disease would be beyond the scope of the present thesis and there are numerous reviews in the literature about this topic (Manuck, 1994). It is of particular interest here to present the main aspects about Type A and the hyperreactivity hypothesis.

Cardiac reactivity is a normal healthy response to stressful situations (Rosenman, 1991b). Problems may arise in people who over-react to even minimal stressful stimuli or when hyper-activation is maintained over long periods of time.

Multiple studies have reported that Type As show hyper-responsiveness to what they perceive as cognitive stressors and challenging tasks and that this hyper-responsiveness could be the link between Type A and high blood pressure and also CHD (Contrada \& Krantz, 1988; Elliot, 1988). Very briefly, it is believed that Type A characteristics are related to a higher blood pressure response and lower blood pressure variability and that a maintained physiological response may lead to some structural damage of the coronary arteries through an increase in plasma catecholamins leading to the formation of atheromes and subsequent arterial narrowing (Abbot \& Sutherland, 1991). This view, however, has met with some opposition (Rosenman \& Rose, 1988), Rosenman, 1991b). Rosenman \& Ward, 1988) point out that the notion of a "hot "versus a "cold" reactor may be an oversimplification of the problem and that there are a variety of methodological problems leading to controversial findings.

Type As perceive challenge in many situations in life, both in and out of the work. This challenge leads them to an over-activation of their cognitive and behavioral systems. In order to attain their ends they over-exert themselves. Powell, Simon, Bartzokis, Patillo \& Thoresen (1991) found that those who became emotionally aroused during the Videotaped Structured Interview for Type A had a 2.3 higher incidence of cardiac death.

Type As are also over-concerned about time and there are an important number of studies in which it was found that time pressure may lead to important changes (i.e. increases) 
in cholesterol level, blood pressure, heart rate, and even ischemic wall-motion abnormalities in the hearts of cardiac patients (for a review see Ulmer \& Schwartzburd, 1996).

Although not directly related to hyper-reactivity, some very interesting evidence concerning changes in ECG patterns in Type As can be derived from the study by Perini, Nil, Bolli, Battig \& Buhler (1993). They used the SI to classify 100 healthy working men into Type A, Type B and Type X and the Cardiac Infarction Injury Score (CIIS), an electrocardiographic technique, to assess ischemic changes which are predictive of cardiac events. Their results showed that asymptomatic Type As had more electrocardiographic changes than Type Bs and, thus, were at greater risk for ischemic heart disease and possibly cardiac death. No differences were found between smokers and non-smokers, age, blood pressure, or family history groups.

There are still a number of issues to be resolved and one of the most intriguing is the timing of the hyper-reactivity. Do Type As have a higher basal level to begin with and are usually in a hyper-reactive state, or is it more a matter of frequent, tonic changes? If so, how long do these changes last?. Is self-report of Type A (and anger) a good predictor of blood pressure changes compared to the SI results or even the information provided by those observing the everyday behavior of that person? Some of these issues are addressed in the studies described in Chapters 2 and 3.

\subsubsection{Culture and environment}

Once beyond the idea that biology in itself cannot provide all the answers and that psychosocial factors may largely, and in interaction with biological aspects, contribute to CHD, other relevant issues must be addressed.

Perhaps one of the aspects that, until recently, has been highly neglected within the psychoscrial risk factors for CHD literature is that of the role of cultural and geographical diversity. With specific reference to Type A, the possibility that this behaviour pattern is not always associated with cardiac atherosclerosis in all cultures but only when certain demands are present has also been proposed (Steptoe, 1981). He further adds that the incidence of Type A behaviour differs between populations suggesting that eliciting stimuli in the individual environment are essential in order to evoke that behavioural style.

The stability of the Type A behaviour pattern across environments, the dependency on specific circumstances or demands, the consideration of the consequences of those behaviours when confronted by different external pressures and the coping styles associated 
with specific challenges, are some of the issues deserving attention both to contribute to a conceptual clarity, and to aid in the assessment of Type A as a risk factor for CHD.

Type A is primarily a "born in the USA" concept. As was explained earlier, its presence probably responded to the needs of a powerful society where each individual felt the pressure to achieve more everyday. It is as if the efforts and gains of the USA as a nation were reflected at an individual level in Americans. Type A was never considered as a stable personality trait by its founders (Friedman \& Rosenman, 1959), but as a pattern of overt behaviour exhibited to cope with an environment perceived as challenging. Still, all research on Type A uses measurement procedures which do not take into account its possible variability depending on factors such as culture, economic and political circumstances or socio-economic status. It is rarely evaluated taking into account the interactions with environmental influences (Blumenthal et al, 1987; Karasek, 1990).

Many countries in the world are following the American socio-economic model. Nevertheless, culture, traditions, rearing practices and even everyday individual and social routines could introduce variations in the structure of concepts such as Type A making some aspects of it (e.g. time-pressure, anger expression, impatience, goal achievement) more frequent, or more risky, or related to different challenges. As expressed by Bracke \& Thoresen (1996), Type A is a culturally created and socially sanctioned lifestyle. It seems likely that social praise and sanctions will lead to different behaviours or lifestyles in different societies. All Type A characteristics may be present in all individuals regardless of their culture but the combination of its components, the prevalence in different groups (e.g. males and females), the challenges for, say, more time pressure or need of achievement, surely are a function of particular (local) cultural and historical circumstances. Thus, it seems necessary to pursue investigations in other cultures to establish the possible universality of global Type A and each of its separate components, as well as their predictive value as risk factors for CHD as it will be addressed in this dissertation.

The picture becomes even more complicated as within the same cultural setting, environmental and social challenges may vary from one arena to another. Work, home, recreational spaces, or the street hazards, can pose different urges or influences in the individual leading to the emergence of Type A behaviours with more or less stability.

By assessing presence of Type $A$ in different settings the stability of the concept may be evaluated. In general, people are classified as either As or Bs as if these were static behavioral traits rather than considering the possibility of situational or environmental variables having an effect in the quantity or quality of display of those behaviour patterns. Conceptually, Type As react when encountering certain kinds of situations which pose a 
threat to their self-esteem, or challenge or involve difficult problem solving (Smith, 1989) and is considered an environmentally provoked syndrome (Suls \& Sanders, 1989). Also, it may reflect the need to avoid the anxiety derived from existential dilemmas (Bracke \& Bugental, 1995). But the consideration of the environment in which these challenges may occur is not included in most of those conceptualisations.

It is proposed here that such a transactional model should be adopted that will emphasise more the differences in Type A behaviour, (e.g. anger manifestation and perception of social support) at home and at work. It is believed that the same type of stressors under different environmental circumstances will pose different demands and therefore different behavioural, and perhaps physiological, responses.

On the other hand, Type A behaviour has been related particularly to the work environment whereas family demands and other interpersonal relations may also represent threats or challenges relevant to the Type A way of responding. (Eysenk, 1991). Work demands tend to intensify the Type A characteristics, especially time urgency, and competitiveness, and have been considered as a particularly appropriate environment to carry out research into cognitive/perceptual differences between Type As and Bs (Phillips, Freedman, Ivanicevich \& Matteson 1991). In addition, comparisons between the work and other environments with respect to the appearance of Type A characteristics seem most relevant in this regard.

Self-appraisal and goal setting are aspects that have received attention in relation to organisational settings (Strube, 1987; Phillips et al, 1991). It is believed that Type As, due to their characteristics, and especially because of their need for self-appraisal and social comparison, react differently than Type Bs in the presence of particular goals. It is assumed in these conceptualisations that the work environment provides a substrate different to other situations where certain responses will occur in terms of their specific demands. Other surroundings, eg home and other social settings, may or may not bring about responses or behavioural patterns similar to those.

\subsubsection{Type A behavior and gender}

What used to be rare in the literature on psychosocial (and biological) risk factors for CHD, namely gender issues, is now becoming increasingly comnon. The notion that prevalence of CHD risk factors, emotional expressions, and culture may be gender specific has been the basis for a growing number of reports. 
In relation to biological risk factors, it has been reported that use of oral contraceptives combined with current smoking, high blood pressure, elevated blood lipids, and menopause are among the most influential in women whereas oestrogen replacement therapy is a protective factor (Brezinka \& Padmos, 1994).

In an additional review study about psychosocial factors of CHD in women, Brezinka \& Kittel (1995) report that low social class, low educational level, double roles of work and family, chronic "troubling emotions" and lack of social support emerged as the most frequent risk factors for women. Most of these seem to be shared with men with the probable exception of multiple roles, which, to the authors' knowledge, have not been previously evaluated in males.

Regarding "troubling emotions", the 8-year follow-up of the Framingham Study (Haynes \& Feinlieb, 1980) showed that women who developed CHD during that period had higher scores on suppressed hostility, tension and anxiety scales than those with no CHD. After controlling for the traditional risk factors, Type A and a measure of "anger-in", were independent predictors of CHD. Nevertheless, at the 20-year follow-up tension and infrequent vacations were independent risk factors of fatal and non-fatal CHD. These results suggest that the predictive power of these particular risk factors is time dependent.

Other studies report somewhat opposite findings. Men and women were not found to differ in anger-in or anger-out in a study with a diverse sample of adults at middle age. They did differ, however, in the likelihood of discussing anger and expressing anger through physical symptoms (e.g. headache when angry), with women having higher scores (Thomas, 1989). Thomas \& Williams (1991) replicated these findings in a study with 720 college students (evaluating anger with Spielberger's Trait Anger scale and the Framingham Anger Scale). Differences in assessment procedures and samples could account for those controversial findings.

The study by Wright. Abbanato, Lancaster, Bourke \& Nielsen (1994) illustrates the gender differences in Type $\mathrm{A}$ and its components, and in anger expression styles. They found that men scored significantly higher on "anger-in" and lower on "anger-control" but that there were no gender differences in "anger-out". Men also had higher scores in a measure of interpersonal control. Males and females did not differ in any of the other sub-components of Type A behavior. Based on their results Wright et al., argue that women achieve their Type A status through different routes. This is a plausible interpretation deserving further attention. The role of cultural aspects may be of great relevance in this respect.

Friedman, Fleishmann \& Price (1996) argue that it is not possible to estimate the prevalence of Type A in women as it may greatly differ from one group of women to another. 
They ascertain that women from a well-developed country will have a different Type A prevalence than that of a Third World nation but they neither suggests the direction of this difference nor give any argument as to why women should differ from men.

Given the fact that increasing numbers of women often occupy two or more roles in almost all industrialized societies, and that in many cases they may feel the need to excel in each of them, Type A characteristics may help them achieve the multiple goals that society and culture have "imposed" on them. The simultaneous effects on health of being employed and being a homemaker was explored by Weidner, Boughal, Connor, Pieper \& Mendell (1997) in the USA. They found that employed women reported less hostility (as assessed by the Hostility sub-scale of the Symptom Checklist (SCL-90)) and lower cholesterol levels than women homemakers or employed men. No significant differences were found in Type A (as assessed by the Jenkins Activity Survey) between these three groups. Thus, it seems necessary to evaluate prevalence of Type A in different parts of the world, and assess gender differences as social, cultural, and political determinants may impose particular rules and obligations on men and women.

\subsubsection{The use of other informants in Type $A$ research}

The complexity of the Type A anger and hostility constructs has been widely acknowledged and this has brought about the problem of multiple assessment procedures related to different aspects of those concepts (Barefoot, 1992). The need for observational methods or observational biographies when assessing negative affect has been suggested due to the problems with self-report measures that may be biased by self-presentation and the limits of self-knowledge or by social desimbility (Friedman, Tucker \& Reisen, 1995; Kneip, Delamater, Ismond, Milford, Salvia, \& Schwartz, 1993).

Spouses are generally expert observers of everyday interaction styles of their partners when they are confronted with potentially anger-arousing situations. There exist in the literature a few reports in which spouse ratings have been used to assess differences between self-reported Type $A$ behaviours, anger, hostility, and associations with disease outcomes in their partners. In 1988, Condon evaluated 41 couples where the husband had very recently suffered an $\mathrm{MI}$ or chest pain or had been diagnosed with a positive coronary angiography and found that wives' reports did not differ from self-reports of Type A. They attributed these findings to possible communication between the spouses when responding to the questionnaires although they were explicitly requested not to communicate. In a later study, Atchinson \& Condon (1994) compared coronary patients and matched controls and 
found that spouse ratings of TABP discriminated between coronary patients and matched controls but self-ratings of patients did not.

In both studies, however, participants were hospitalized coronary patients, there may have been selection bias due to drop out, and, finally, in the latter study, patients knew beforehand that their spouses would be replying to the same questionnaires as they themselves did.

Spouse-ratings of hostile outlook and anger-in have also been found to predict CHD better than self-reports. In the study conducted by Kneip et al., (1993) spouses rated their male or female partners who were just about to undergo a tallium scan and found that only spouserated hostility contributed to the prediction of CHD status. They also found interesting gender differences in spouse reports but not in self-reports indicating that women rated their husbands higher in anger-out than men rated their wives.

It seems interesting to pursue this line of methodological guidance and include spouse ratings in risk factor studies. Obviously, methodological limitations will have to be overcome. It would be convenient that neither participants nor spouses would know that they will be requested to provide information about their partners until the moment of receiving the questionnaire. The need for strictly independent responding must be strongly emphasized. It would also be important to assess the agreement or disagreement levels between both types of report and the associations with some cardiovascular health indicators. Moreover, possible gender differences should also be explored.

\subsubsection{Type A behavior and Social Support}

Although it does not constitute a major focus of attention in this dissertation, some attention will be given to Type $A$ and its relation to social support in some of the following chapters.

Social support has proved to be a protective factor for CHD in an important number of studies (Seeman \& Syme, 1987; Orth-Gomér \& Unden, 1990; Orth-Gomér, Rosengren, and Wilhemsen,1993)

The conceptualization of social support goes beyond the scope of this work. We will limit ourselves to state that it may refer to structural (i.e., partner, family, friends, colleagues, sports partners, etc) and functional (i.e., quality of those relationships and their actual use) networks around an individual (Seeman \& Syme, 1987).

After the analysis of five prospective studies (with follow-up episodes from 8 to 13 years) and with data from over 37000 participants from the USA and Europe, House, 
Landis \& Umberson (1988) concluded that lack of social support (mainly structural) was a strong risk factor comparable to that associated with cigarette smoking, this strongly indicating the importance of social networks in relation to cardiovascular health.

Quite obviously, the functional aspects of social support require time, interest, and motivation from the person. Type As are so preoccupied with accomplishments and are so much committed to work that they may disregard their social networks and will not make time to nourish their relationships. In fact, denial of the need of other persons has been shown to be part of Type A characteristics (Matthews, 1982).

Several studies have explored the relationship between Type A and social support. In Finland (Raikkonen, Keskivaara \& Keltinkangas-Jarvinen, 1992), 1296 young adults were asked to report about their Type A behaviour (Finnish questionnaire) and social support networks (Perceived Social Support Scale-Revised-PSSS-R). They also responded to a three-item hostility scale. Results showed that the associations were dependent on gender and that they were only significant when considering the multidimensional nature of the constructs but not with the global scores. Competitiveness and irritability were related to low levels of social support and engagement-involvement was related to high level of perceived social support. When examining results according to gender, the authors found that very hard driving, competitive and non-involved Type A women with extreme hostility perceived the lowest social support, while the opposite was true for men. This is a most surprising result attributed by the authors to gender-specific cultural expectations

Studies like the ones just described illustrate both the importance of social relationships in relation to health and the relevance of socio-cultural determinants in the specificity of psychosocial risk factors for CHD. There is an urgent need to further evaluate social support in relation to other psychosocial risk factors and to explore possible environmental influences in the interaction of social support and Type $A$ in their relationship to blood pressure and other CHD risk factors.

\subsection{AN OVERVIEW OF VITAL EXHAUSTION AND CHD}

\subsubsection{Origins and definition of Vital Exhaustion}

As was the case with Type A, the term Vital Exhaustion (VE) emerged from clinical observations of candiac patients in an attempt to clearly define the symptoms of MI patients that preceded their cardiac events. In cardiological studies, chest pain, dyspnoea, 
and fatigue are the most commonly reported precursors of $\mathrm{MI}$, reported by $10 \%$ to $60 \%$ of patients. More specifically referring to symptoms of "undue fatigue" or "lack of energy", reports state that these were found in $30 \%$ to $56 \%$ of people before a heart attack (Appels \& Otten, 1992). The aetiology of these symptoms of fatigue is not yet clear. They have generally been attributed to sub-clinical atherosclerosis, myocardial ischemia or to medication side-effects (Appels, 1990). In addition, fatigue can be attributed to anxiety, emotional tension, and depression (Appels \& Otten, 1992). Cardiologists have mainly attributed these feelings to cardiac problems but there are only few cardiological studies investigating the origins of those feelings before the cardiac event. Nor have they tested either how many patients may remain exhausted after CABG or PTCA (Appels, 1990).

During a large series of interviews with candiac patients and their wives, Appels, Höppener \& Mulder (1987) reported that it was common to hear expressions such as "The well is drying up", "My body is like a battery that is losing its power" referring mainly to problems they had experienced in life. The wives mainly expressed that there had been obvious increases in irritability in their husbands in the months previous to hospitalization.

The term "Vital Exhaustion" emerged in an effort to define as accurately as possible the mood and feelings that precede MI. The literature is rich in studies where depression is found to be present in varying periods of time either before or after a heart attack (see. Fielding, 1991 for a review; Barefoot \& Schroll, 1996). Nevertheless, the most commonly reported symptoms of cardiac patients do not fully correspond to depression as a psychiatric illness, that is, as described by the DSM-III-R or DSM-IV criteria (see later section in this chapter). Vital Exhaustion has been defined as "a state characterized by feelings of umuswal tiredness and lack of energy, increased irritability, and feelings of demoralization" (Appels, 1997). It is a state of physical and mental exhaustion. Other symptoms frequently reported by patients are loss of vitality, listlessness and loss of libido (Appels \& Mendes de Leon, 1989). Loss of sleep and sleep disturbances has also been reported to be part of VE previous to MI (Falger \& Nelissen de Vos, 1997).

Having established that this type of gloomy symptoms is present in an important number of patients before the onset of $\mathrm{MI}$, research in the area has had five main objectives. First, to clearly ascertain the components and characteristics of those feelings (e.g. are they the same as in, or how similar are they to, depression?). Second, to establish their possible. aetiology: (e.g. can they be attributed to physiological cardiovascular changes, like decrease in cardiac output resulting from ischemia, or to age and angina?, are they the result of medication (beta-adrenergic blocking, agents and diuretics)?, do they have a more psychological aetiology, emerging as a result of probably long-lasting emotional tensions or 
some other psychological condition?, or are they the result of a combination of all these?. Third, to create and refine appropriate assessment procedures (i.e., questionnaires, interviews). Fourth, to assess the relationship of VE with other psychosocial risk factors (e.g. is VE related to Type A, to hostility and anger, to job stress and negative life-events?), and fifth, to establish its construct validity and stability across cultures and social contexts.

\subsubsection{Vital Exhaustion: The evidence}

As previously mentioned, the history of research in Vital Exhaustion is relatively short. In fact it only goes back as far as, 15 years. Research in which VE has been evaluated is summarised in Table 1. In a large prospective study set up in 1979 - the Rotterdam Civil Servants Sudy (RCSS) (Appels \& Mulder, 1988) - 3877 male civil servants aged 39-65 years old were screened for cardiovascular risk. The screening included the Maastricht Questionnaire (MQ) with 37 items asking about manifestations of exhaustion, emotional drain, irritation, depression, and sleep problems (MQ-Form A). In addition, 21 items derived from clinical observations were added. After a follow-up period of 4.2 years, 59 participants had experienced an MI. The MQ was significantly associated with an increased risk of future MI after controlling for age, smoking, cholesterol, and blood pressure and, interestingly, this risk showed a decrease as the follow-up period increased. That is, in the first most recent year the relative risk was 10.05 , while for the remaining years it was $2.23,3.04$ and 0.68 , respectively indicating that VE was a short-term risk indicator for MI (Appels \& Mulder, 1988). Many of the items in the MQ corresponded to what would be described as "depression" according to the then most recent DSM-III criteria. Yet, the authors could not simply conclude that "depression" was a risk factor for MI because there were important differences between the two constructs, depression, and VE, mainly in relation to guilt feelings and self-esteem. This crucial issue will be discussed later in this chapter.

Besides this prospective study with healthy participants at entry, another study to test the predictive power of VE was conducted with 127 patients who were about to underga a Percutaneous Transluminal Coronary Angioplasty (PTCA) procedure (Kop. Appels, Mendes de Leon, Swart, \& Bar, 1994). Patients responded to the MQ two weeks after hospital discharge. After a follow-up period of 1.5 years $35 \%$ of the 43 exhausted patients at entry had experienced a new cardiac event compared to $14(11 \%)$ of the not-exhausted patients had a cardiac event. The significant difference $(p=02)$ between the exhausted and non-exhausted patients remained after controlling for the traditional risk factors. Most relevant was the fact: 
that the predictive value of VE was independent of the severity and extent of the coronary vessel disease or any other clinical characteristics.

A number of case-control investigations were also conducted to assess the discriminative power of VE in various patient groups. Mendes de Leon (1988) conducted an investigation in the USA with a small sample of heart patients $(\mathrm{N}=22)$ with symptoms (e.g. chest pain) that were suggestive of unstable myocardial ischemia. Controls $(\mathrm{N}=44)$ were also hospitalized patients but suffering from ailments unrelated to the cardiovascular system. Cardiac patients had higher, but not significantly so, VE scores. Nevertheless, when VE scores were divided into tertiles, those in the upper tertile had an OR of 2.36 , which was very near significance level $(p=.07)$ indicating that the risk of developing unstable AP in that particular group was more than two fold compared to those in the lower tertile. The author argued that the reasons for not reaching a clearly significant level may have been related to the small sample size and to the different formats of the question and answering categories used in the English version of the $\mathrm{MQ}$.

In a Dutch study with a substantially larger group of participants, Falger, Schouten, Appels \& De Vos (1988) compared the prevalence of VE symptoms between a group of 133 male cases with first MI, 192 aged-matched male hospital controls, and 133 aged-matched male neighbourhood controls (sleep complaints and other possible behavioural characteristics were also compared). MI patients were asked about VE in the last six months previous to their cardiac event, hospital controls about the same time previous to their hospitalization and neighbourhood controls about the six months previous to responding to the questionnaire. Results showed that the mean MQ scores for the MI patients (15.4; S.D.=9.5) were significantly higher than the mean scores for both hospital $(10.0 ; \mathrm{S} . \mathrm{D}=8.5 ; \mathrm{p}<.001)$ and neighbourhood controls (7.8; S.D.=8.5; p<.001). This was after eliminating the sleep complaint items from the $\mathrm{MQ}$ which, on their own, also significantly discriminated between the groups. In fact, VE was a confounder with respect to sleep complaints that were the variables that most clearly distinguished between the MI cases and both control groups. In this study, the fact that the mean MQ scores were significantly different from those of the hospital controls ruled out the possibility that the VE differences were due to retrospective bias as a result of recent hospitalization.

Similar findings were reported in a smaller study with women ( $79 \mathrm{MI}$ cases and 90 aged-matched hospital controls) where mean VE scores of MI cases (20.6, SD=11.9) were significantly higher that those of controls $(17.0 ; \mathrm{SD}=11.2)(\mathrm{t}=2.02 ; \mathrm{p}=, 04)$. In this study, patients were also divided into an "exhausted" an a "non exhausted" taking the median as the 
cut-off point. It was found that $63 \%$ of the cases and $39 \%$ of the controls had been "exhausted" before admission to the hospital (Appels, Falger \& Schouten, 1993).

The latest published case-control study reported is that by Meesters \& Appels, (1996h). They compared 81 male first $\mathrm{MI}$ patients and 168 age-matched healthy neighbourhood controls and found that both the MQ and a newly developed interview schedule to measure VE (the "Maastrich Interview for Vital Exhaustion", MIVE, see below) discriminated well between cases and controls according to univariate and multivariate anallysis.

In a recent study, Kop, Hamulyak, Pernot: \& Appels (1998) used the MIVE to further assess a group of individuals who had earlier been assessed with the $\mathrm{MQ}$ and compared 15 exhausted and 15 non-exhausted participants for fibrinolytic and coagulation indicators. The purpose of this study was to look at the effects that prolonged stress may have had on coagulation factors and fibrinolytic capacity. Results showed that the exhausted participants had higher activity levels of PAI-1, suggesting a reduced fibrinolytic capacity in the exhausted.

In summary, with few exceptions, published reports show that VE seems to be a short risk factor first MI and for recurrent coronary events after successful PTCA. Nevertheless, there are an important number of issues yet to be resolved. These issues include assessment aspects, conceptual delimitations and clarifications, universality or cultural stability, links with CHD, and gender differences. Some of these issues will be dealt with in this dissertation (Chapters 5 and 6).

\subsubsection{The measuring instruments}

As is usually the case with psychosocial risk factors, evaluation procedures represent a crucial issue. In the case of VE the instruments to assess its presence or absence were developed along with the first studies and, with the objective of improving their validity and reliability, have been undergoing changes until very recently.

The first questionnaire to measure VE was the Maastricht Questionnaire -MQ- Form At, which was used in the IMIR study (Appels, 1983). An item analysis of that first version showed that 16 out of the initial 37 items contributed significantly to the predictive power of the questionnaire and that 8 of the added 21 in the RCSS study were also predictive of AMI. With these items the MQ-form B was created containing 21 items after three were removed due to unclear content validity (Meesters \& Appels, 1996a,b). 
These 21 remaining items were (abbreviated form):

\section{Often tired}

Have trouble falling asleep

Often wake up during the night

Feel weak

Feel as though I am not accomplishing much

Have difficulties coping with everyday problems

Believe to have come to a "dead end".

Feel listless

Sexual interest diminished

Feel hopeless

Have difficulty in grasping a new problem

Easily irritated

Want to give up trying

Feel fine (no)

Body is like a battery that is loosing its power

Want to be dead at times

Just dont have what it takes anymore

Feel dejected

Feel like crying

Wake up exhausted

Have difficulty in concentrating

Have strange bodily sensations

Shrink from regular work

Have spells of shaking and trembling all over

Most of these items were shown to be associated with stable relative risks, of -on average- about 2.00 (Appels, 1990). To respond to the questionnaire the person had to answer one of three possible categories: Yes $=$ this VE symptom is present; $N o=$ this VE symptom is not present; or "?" = I am not sure about the presence of this symptom. In the RCSS study the decision about presence or absence of vital exhaustion was taken by computing a cuttingpoint that was one Standard Deviation (8.67) above the mean (8.82) score. Thus, exhausted participants were those with a score of 17 and above.

This questionnaire has been used in almost all of the longitudinal and cross-sectional studies assessing the association of VE with AMI that were reported in Table 1. Nevertheless, 
questionnaires as instruments to collect precise psychosocial information have many drawbacks that range from misunderstanding of item contents to time scale confusions or social desirability issues. Moreover, In the specific case of the $M Q$, it has been pointed out that the reports of symptoms of tiredness and fatigue may be due to a general tendency to complain or to prolonged overwork (Meesters \& Appels, 1996a). A pilot study previous to the Van Diest \& Appels (1991) report illustrates specifically some of the shortcomings of the MQ. They found that, after interviewing 15 individuals who had been previously classified as exhausted according to the MQ. 6 had not been properly included as they were individuals with a chronic fatigue condition.

With the objective of overcoming some of these limitations, Meesters \& Appels (1996a) constructed an interview schedule,(Maastricht Interview for Vital Exhaustion, MIVE) which contained items similar to those on the MQ but which differed in administration and scoring procedures. Based on of the predictive power of the items in previous versions of the questionnaire (Form A, Form B), some items were removed, others were split into two statements and a new one was added. Thus, the final version contained 23 items. During the administration of the MIVE each question was always followed by another question about the time period during which the symptom had been present, scoring it as "yes" if it had been during the past 18 months, "no" if it had not been present and "?" if the interviewer was in doubt. The items with a "?" are repeated at the end of the interview giving the interviewee the chance to come back to the issue. If it is not possible to ascertain its presence this second time round, then it is finally scored as "no". During the interview, the interviewer thus has the opportunity to explain the meaning of each question in detail. This method of administration remarkably reduces the possibility of false positives in the respondents" answers. The authors provided an Appendix with specific instructions about the way in which the interview should be administered and scored.

In that same case-control study the discriminative power of the MIVE was tested by comparing 81 male first AMI cases and 168 age-matched healthy neighbourhood controls. The MIVE was also compared to the MQ. Results showed that the items about tiredness, irritability and annoyance were the ones most frequently endorsed and that all complaints except four were more frequent among cases. After discarding those participants who had had complaints about exhaustion for more than 18 months, $40 \%$ of the cases and $8 \%$ of the controls were considered to be "exhausted" before the AMI or before interviewing, indicating good discriminative power of this interview. When comparing it with the questionnaire it was found first, that the questionnaire yielded $23 \%$ false positives compared to the MIVE and second, that, although both instruments discriminated well between cases and controls, the 
discriminating power of the MIVE form was considerably higher than that of the questionnaire $(\mathrm{p}=.02$ and $\mathrm{p}=.000$, respectively). Logistic regression analyses showed similar results (OR=9.50 and 2.26, respectively).

These findings clearly indicate that the MIV'E is to be preferred over the questionnaire when assessing Vital Exhaustion and its use should be strongly recommended. Nevertheless, as it is undeniable that questionnaire administration is a much more economical and simpler method of data collection, some further testing is necessary to affirm the differences between the two forms of administration. It is proposed that the same versions of interview and questionnaire should be compared, changing only administration and scoring procedures (Chapter 5).

In a subsequenit report, (Mesters, 1996b) the MIVE was shown to have good reliability ( $\alpha=, 90$ in the AMI cases group and $\alpha=, 86$ in the control group). The interview also showed to have good validity (an OR of 8.11 after controlling for angina and the classic risk factors, age, smoking, and family history of CHD). Moreover, VE according to the interview was found to be positively associated with TABP and with two dimensions of hostility. Four MIVE items had factor loadings below . 40 and three items did not discriminate between cases and controls. Nevertheless, the authors decided not to remove them from the final item pool as they had shown strong predictive values in a previous prospective study.

Although some limitations were acknowledged (e.g. interviews had not been scored blind, no control for sub-clinical heart disease, and the retrospective nature of the study), the authors concluded that the MIVE was a reliable and valid measure to assess VE in population studies.

\subsubsection{Vital Exhaustion: Type A, Hostility, and Anger expression.}

In contrast with Type A, which is a long-term risk-factor for CHD, VE is considered to be a short-term risk factor that can be: observed a few months before the cardiac event will occur. It has been proposed that VE may be in fact the psychological end-product of Type A behaviour throughout a life time. As early as 1977, Glass proposed that Type As in the long run, will become exhausted as a result of their excessive need of control of life events that are perceived as stressful or challenging.

This is a reasonable hypothesis and somehow related to the hyperactivity proposal discussed earlier, which stated that Type As unnecessarily over-react, both physiologically 
and psychologically, to everyday stimuli and will maintain that over-stimulation over longer periods of time than Type Bs.

Type As attentional strategies indicate their need to excel. This has been demonstrated by De la Casa, Gordillo, Mejías, Rengel \& Romero (1998). They found that Type As when confronted with a dual task, in which there was ambiguity between the main and the secondary tasks, tried to process as much information as possible from both. That is, they tried to respond to the main task but at the same time keeping as much information as possible from the second one for latter purposes. This can be labelled as "hypervigilance". It could be argued that this sustained attention requires high concentration efforts and may be very effective in the short run. Moreover, keeping this style throughout a life-time, together with a need for control, chronic work overload, and need of achievement, could be held responsible for some kind of "psychological fatigue". Unfortunately, there are only few studies in which the combined effects of TABP and exhaustion have been evaluated.

Falger (1989) tested the hypothesis that exhausted Type As will be at elevated risk of AMI compared to non-exhausted Type As. He found that the combined effect of both factors was greater than the single effect of each of these factors (which were also significant). It was concluded that "a state of exhaustion modifies the risk for future myocardial infarction associated with Type $A$ and vice versa". The results of this investigation are most important in terms of long and short-term risk for MI, as it offers the possibility to identify individuals who may have experienced fatigue due to some chronic ailment that has to be differentiated from VE as precursor of AMI. However, in this study the MQ was used for the evaluation of VE and we have already discussed its limitations. So far, only few replications of this study, i.e. another PhD dissertation (Nielssen-de Vos, 1994) and Meesters \& Appels (1996b) have confirmed those findings.

In the study by Meesters \& Appels, (1996b) some methodological limitations were overcome by using the MIVE and SI in the assessment of VE and TABP, respectively. As mention 3 ed before, they compared 81 first AMI patients and 168 neighbourhood controls and found that Type A participants reported significantly higher levels of exhaustion than Type B subjects and that this was more so in the group of AMI patients than in controls. Unfortunately, only global Type A was assessed and it was, therefore, not possible to look at the relationship of VE with Type A sub-components in order to see whether VE was associated with different Type A characteristics both, qualitatively and quantitatively..

In a sample of Finnish middle-aged men, Keltinkangas-Jarvinen, Raikkonen \& Hautanen (1996) examined the role of Type A and VE in the neuroendocrine response as related to cardiovascular functioning. Exhaustion was assessed by means of the MQ and Type 
A by means of the Jenkins Activity Survey. The results were not very clear-cut and rather difficult to interpret even for the authors themselves. They suggest that both, VE and Type A contributed independently to the observed variations of the hypothalamic-pituitary-adrenal axis (HPA) hormones. In fact, there was an interaction between Type A and VE, in that the combined effects of Type A and VE were different (sometime opposite) from those of Type A alone. They also found that only the "Speed and Impatience" sub-scale of the JAS contributed significant to the hormonal response. Thus, they concluded that Type A does not necessarily lead to exhaustion and fatigue but that only some Type As, perhaps those with more impatience, will become exhausted in the long run and, thus, are the ones at higher risk. Again, these results should be interpreted with caution as both instruments, the MQ and the JAS, have received important criticisms.

It has been argued that the strong achievement striving of Type As may be in fact a response to an underlying unconscious depression and that after a life-threatening event that makes this incessant activity come to a halt, such as an AMI, the depression becomes "unmasked" (Allan \& Scheidt, 1996). These authors continue to argue that, from a psychoanalytic point of view, depression may be in fact "anger tumed inward" and therefore, suggest a strong association between hostility, a crucial component of Type A, and depression.

Although not referring directly to Vital Exhaustion as it has been conceptualized here, Ulmer and Schwartzburd (1996) offer a alternative proposal about the connection between time pathologies and exhaustion. Time pathologies refer to "the toxic continuum of disordered behaviors, perceptions, and states running the gamut from "mild time urgency" to "severe hurry sickness" (p.332). From their clinical experience the authors infer that for some cardiac patients the state of exhaustion that they report may be the result of chronic time-urgency and the ensuing exhaustion may constitute, then, a risk factor in itself rather than being a result of the ongoing disease process. They argue that "time urgency" and its progress to "hurry sickness" is the result of interactions between physiological, psychological and sociological problems involving cultural patterns (in the United States). These cultural patterns have evolved into a very strong belief system, contemplating that an individual's worth depends on as many accomplishments in as short periods of time as possible.

One of the main cornponents of the Vital Exhaustion syndrome is "increased initability". In the Meesters \& Appels, (1996a,b) studies hostility was measured by using both, the Cook-Medley Hostility $(\mathrm{Ho})$ Scale and the Buss-Durkey Hostility Inventory (BDHI). Exhaustion was positively associated with some dimensions of hostility, but not with 
others, and the associations were different for AMI cases and for healthy controls. In both groups VE was significantly, and strongly, correlated with "irritability" and "resentment". On the other hand, "Assault" and "Indirect Hostility" were strongly correlated in AMI cases but were only weakly significant or not significant at all in controls. In general, correlations were stronger in AMI cases. These results indicate that hostile cognitions and affects may accompany exhaustion but that the patterns of association may be different for those who recently suffered an MI compared to those who did not. It would be of interest to investigate the anger-expression styles in relation to exhaustion, looking at the behavioural manifestation of this negative affect state.

This is an area that definitely needs more attention in the future given the relevance of investigating behavioral and psychological patterns that may lead to exhaustion in the long run.

\subsubsection{Vital Exhaustion and Depression}

Reports about the role of depression in CHD have been published in the scientific literature since 1960. An excellent review by Fielding (1991) gives an account of those early studies in which associations between depression and CHD have been found acknowledging. at the same time their methodological shortcomings. He also describes later prospective and retrospective studies where, more or less successfully, depression or depression-like states were found to precede or follow cardiac events. The well-know meta-analytic study about cardiovascular risk factors and CHD carried out by Booth-Kewley \& Friedman (1987) also revealed that depression was a very strong predictor for CHD, more so than global Type A. Dimsdale (1993) later replicated this finding in women who showed co-morbidity between depression and CHD. More recently, the number of studies about the relationship between depression and MI has increased (e.g. Anda, et al, 1992; Frasure-Smith, Lesperance \& Talajic, 1993; Barefoot \& Schroll 1996).

One of Fielding's (1991) conclusions is that there is enough evidence to support the notion that a depressive-like state is associated with AMI but that some negative results merit further investigation in the area. Moreover, he points out that the "varied criteria for "depression" in the studies makes interpretation more problematic".

Since the first reports showing Vital Exhaustion to be a risk factor for CHD, the controversy about its similarity or difference to depression has been treated The initial clinical interviews that led to the formulation of the construct of VE revealed that cardiac patients reported having experienced symptoms that included both, fatigue and a negative 
mood state for a few months previous to their cardiac event. These symptoms are usually important parts of a depressive disorder thus, it is not universally accepted that a distinction between VE and depression would be useful (Fielding, 1991).

While it may be accepted that VE and depression share important common characteristics, there are crucial elements of depression that are not generally present in vitally exhausted individuals. When asked to describe their emotional state previous to their MIs, patients commonly report fatigue, increase imitability, loss of libido and demoralization but they generally make little reference to low self-esteem or guilt feelings (Van Diest \& Appels, 1991). They do not attribute those feelings to their personal failures like depressed individuals would, but to external circumstances, like negative life-events or losses. Thus, there is a similarity with depression-like symptoms but their descriptions do not correspond with a fullfledged depressive state.

The use of different assessment procedures for depression has largely contributed to the controversy. Van Diest \& Apeels, (1991) conducted a study in which negative mood in exhausted and non-exhausted participants was, assessed by self-monitoring for 21 days with the Profile of Mood States (POMS) and retrospectively with the Beck Depression Inventory. $\mathrm{VE}$ was assessed by means of the $\mathrm{MQ}$. They found that a depressed mood was mostly absent in exhausted individuals concluding that VE was distinct from depression.

Terminology is very important and perhaps it should be concluded that "depression" may not be the appropriate term to label the emotional state that precedes AMI. In fact, within the different studies terms such as "disturbing emotions", "emotional upset", "depressive symptoms", and even DSM-III or IV defined Major Depressive Disorder (MDD), are used indicating that there is no consensus as to the precise characteristics of this negative mood state in heart patients. One fact is clear namely that the term "depression" refers to a psychiatric condition that shares some important symptoms with the negative emotional state of cardiac patients, but also that there are crucial dissimilarities that should be taken into account for several reasons, from aetiology to intervention. Thus, it may be said that the construct of VE tries to describe those symptoms in a more precise manner than the term depression itself.

In a recent review, Appels (1997) discusses the numerous questions that can still be posed regarding the association between depressive: symptomatology and CHD. The first issue to consider is that of the precise definition of that depression-like state. One relevant finding concerning this issue is that, in the various prospective studies carried out on $\mathrm{V}^{\mathrm{E}} \mathrm{E}$, the items neflecting a negative mood state lost their predictive power when controlled for items 
enquiring about exhaustion and loss of energy, indicating the relative importance of these two aspects of negative mood-states in general as predictors of AMI.

To conclude, the examination of the relationship between VE and negative mood previous to a cardiac event assessed from various perspectives, and particularly related to a self-deprecatory image, deserves further attention. The associations of exhaustion with frequent thoughts about failure and self-blame would be indicative of a certain similarity between both forms of negative affect. In contrast to this, it would also be interesting to explore the relationship of VE with a positive frame of mind. This is part of the objectives of the studies described in chapters 5 and 6.

\subsubsection{Vital Exhaustion: cause or consequence or both}

As was pointed out before, the concept of VE is rather new and up until now research has been focusing mainly on assessment and predictive issues. More recently, attention has been directed to the possible pathways linking the fatigue, irritability, and demoralization symptoms to CHD. The obvious question to address would be related to causality : Is cardiac disease a product of that disturbed mood and physical condition? or is VE the result of the presence of underlying, as yet asymptomatic cardiac malfunctioning ? However, the possible parhways have to be established first in order to later investigate the direction of the relationship.

From a cardiological point of view the fatigue and lack of energy prior to a cardiac event is the result of the ongoing disease process. Thus, exhaustion is considered a marker of sub-clinical heart disease. This view was disproved by Kop, Appels, Mendes de Leon, de Swart \& Baer (1994) in which it was found that the exhaustion felt by some patients following PTCA was related to the number of diseased vessels but unrelated to left ventricular ejection fraction. Moreover, The predictive value of VE for a future cardiac event was not confounded by the extent of the coronary disease or the left ventricular ejection fraction.

New ideas about the cause of the fatigue feelings and their relationship with CHD have recently emerged. These ideas were summarized by Appels (1997) and originate in the psychoneuroimmunological domain. They are more related to functional than structural (anatomical) aspects of the disease. Very briefly, Appels proposes that prolonged stress may result in the reactivation of the cytomegalovirus (CMV) and the bacteria chlamydea Pneumoniae (CP). These reactivations may cause or aggregate the inflammation of a coronary vessel, leading to increased production of cytokines. Cytokines are known to evoke feelings 
of tiredness and malaise. This hypothesis was tested in a study of 15 exhausted and 15 nonexhausted angioplasty patients. Results indicated that exhausted patients had higher mean antibody titers against $\mathrm{CMV}$ and $\mathrm{CP}$ and higher mean levels of $\mathrm{IL}-\mathrm{I}$ and INF- $\alpha$, indicating that VE is associated with the inflammation process (Appels, Breuggemen, Băr, Bir \& deBaets (1998). The interpretation given by Appels is that increased secretion of cytokines amplifies existing feelings of VE as part of homeostatic reactions to inflammation. These preliminary results are very promising and, at present, other studies in this area are being conducted to further test the above-mentioned hypothesis.

\subsubsection{Vital Exhaustion: cultural issues}

If Type A is a "bom in the USA" concept, it could be said that VE is a "born in The Netherlands"" concept. The Dutch research team, directed by Professor Ad Appels, set out in the 80 's to identify in a systematic mode on the basis of clinical observations the feelings and mood states that patients experienced prior to their cardiac problems. This is somehow similar to what Friedman \& Rosenman did in the $50^{\circ} \mathrm{s}$ after gathering clinical information from cardiac patients and proposing the Type A concept.

The Type A concept has spread throughout most of the psychosocial cardiovascular research in the USA for a few decades and has had an important impact on European research although not as extensive. Vital Exhaustion, a much younger concept, although similar to the "old" depression syndrome, has been exported to other European research teams but has had a relatively low impact in the USA, despite the promising findings in both longitudinal and case-control studies. The reasons for this are not particularly relevant to this report but the issue is raised here to highlight the restrictions that locally based findings have in relation to both, the strength and solidity of the concepts and the universality of their predictive value as risk factors for CHD.

Much more than in the case of biological risk factors, the need to investigate the stability of psychosocial risk factors across cultures and environments is urgent and most profitable in terms of prevention and rehabilitation.

Apart from the conceptual considerations regarding Vital Exhaustion, its aetiology and its predictive power for AMI, other questions such as cultural dissimilarities and universality of this construct could be raised. The possibility exists that due to different patterns of daily routines, to differences in major stressors, or to differences in culturally dictated coping skills, there may also be differences in the type and characteristics of 
psychosocial risk factors. People become habituated to disadvantageous circumstances and what in one culture may be fully accepted may in others lead to exhaustion in a few days in a different culture.

The importance of differences in urban mobility, social class, educational level, marital status, and family culture and lifestyle in relation to subsequent cardiovascular events, was pinpointed in the Kaunas-Rotterdam Intervention Study (Bosma, Otten, Appels \& Sturmans, 1995), a 10-year longitudinal study with about 3000 participants from each of the two cities. Main findings showed that 1) low SES was significantly associated with CHD and total mortality in both cohorts, 2) unmarried participants had a higher risk of non-fatal AMI in The Netherlands and divorced men had a higher risk of CHD mortality in Lithuania but not in the Netherlands, 3) those growing up in small villages and then moving to the two big cities had a greater risk of AMI. In addition, those answering positively to the question "At the end of the day I am completely exhausted mentally and physically" at screening had a much higher risk of CHD in the first years of follow-up but not so much later. Thus, this study illustrates that sociological and cultural aspects are highly relevant in relation to elevated cardiac risk.

\subsubsection{Vital Exhaustion and Gender}

Information about VE in women is scarce. In fact, only one report was found in dealing with this issue. Appels, Falger and Schouten (1993) found, in a case-control study in women that the relative risk for AMI associated with VE was 2.75, after controlling for the traditional biological risk factors. In these women, family and financial problems during childhood and unemployment were related positively to VE. In addition, women with multiple roles, that is, taking care of the home with children under 16 and working outside the home had significantly higher scores on vital exhaustion than those with no household obligations.

The importance of assessing VE in women and comparing them with men is obvious given the increase in morbidity and mortality due to cardiovascular problems in women. Recent evidence about gender differences in sociocultural risk factors between men and women (Jacobs \& Sherwood, 1996) further justify this need. Moreover, the cumulative evidence about the effects, positive and negative, of multiple roles on the health of women suggests that in the long run these may have strong repercussions on their energy and vitality. As was previously mentioned, women may be favoured by having Type A characteristics that: 
will allow them to carry out their everyday roles. In the long run this, however, may lead to exhaustion. Thus, the relationship of Type A and exhaustion in women must be further explored.

\subsection{DESIGN OF THE THESIS}

In this dissertation various issues concerning Type $\mathrm{A}$ and Vital Exhaustion will be addressed through 5 distinct studies. Each of these was conducted in different groups of participants, all working individuals in different organisations. Due to particular circumstances of the author, one of the studies was conducted in England whereas the other four were carried out in Venezuela with a specific interest in evaluating CHD risk factors in a Latin American context.

More than an ordered sequence of reports this thesis will explore diverse aspects of Type A and Vital Exhaustion mainly in a Latin American culture, where little research in this area has been reported in the international literature. All five studies embody an implicit interest in manifestations of psychosocial risk factors in different environmental contexts. Within the Type A concept, its global evaluation and that of its components, especially angerexpression styles, are a major issue in most of the 5 reports. A particular interest in gender differences is also present in two of the studies (chapters 2 and 4). Issues related to cardiovascular reactivity are also addressed in two of these reports (Chapters 2 and 3 ). Indicators of the prevalence of Type A in different occupations in Venezuela are presented in Chapters 2 and 4. The value of other informants, i.e., spouse and work supervisor, regarding Type A and blood pressure is the main purpose of chapters 3 and spouses reports are an important source of information also in chapter 5 .

With regard to Vital Exhaustion research, the validation of the Spanish versions of the two existing instruments, the questionnaire and the Interview, was the first logical step which will be reported in chapter 5 . This is followed by a case-control study designed to explore the discriminative power of Vital Exhaustion a Latin American context (chapter 6 ). In both studies the associations of VE with other psychosocial risk factors, such as Type A and anger-expression, are explored. The prevalence of VE in men and women in a group of Venezuelan employees is established in Chapter 5.

Finally, the main conclusions from these five studies, and recommendations for future investigations, are the objectives of chapter 7 . 


\section{CHAPTER 2}

\section{TYPE A BEHAVIOUR PATTERN AND CARDIOVASCULAR REACTIVITY IN MANAGERS ${ }^{1}$}

\section{2.i. SUMMARY}

The main purposes of this study were, first, to assess the prevalence of Type A Behavior Pattern (TABP) and cardiovascular risk factors, particularly cardiovascular reactivity, in a group of high ranking managers, and, second to assess the associations of TABP and those risk factors in that same group. The prevalence of TABP, heart rate (HR), and systolic and diastolic blood pressure (SBP, DBP) were evaluated during two cognitive tasks in a group of top managers ( 46 men and 6 women) from a large organization. Habits associated with cardiovascular risk (sedentariness, smoking, alcohol consumption, and obesity) and biological risk factors (family and personal history of cardiovascular problems, high cholesterol, and high triglecerides) were also assessed. Results showed a very high percentage of TABP (85\%). As for medical and lifestyle risk factors, Sedentariness, alcohol consumption, and a family history of cardiovascular problems were also quite characteristic of this group. A very high proportion of participants had more than two biological risk factors or habits associated with cardiovascular risk, Type As and Type Bs were significantly different in personal history and hypertension, with As being at more risk. Solving such cognitive tasks as abstract reasoning and mental arithmetic within the work environment produced a significant increase in HR, SBP, and DBP, as well as a subjective measure of tension and this change was associated with level of performance. SBP was significantly higher at the beginning of the experimental session than during the rest periods just before, or between, tasks. HR at the beginning of the session was associated with level of performance. Type As differed in SBP from Type Bs at the end of the session only. These results indicate the importance of taking not only the resting or performance measures into account but also those at the beginning and at the end of an experimental session, i.e., before and after experimental manipulation. The small number of Type Bs in this group did not allow further conclusions to be drawn about their differences with Type As. 


\subsection{INTRODUCTION}

In the mid 1950s, the role of traditional risk factors - cholesterol, hypertension and smoking - in the development of coronary heart diseases (CHD), started to be called into question since these factors could not account for either the rapid increase in the incidence of CHD in the twentieth century or its geographical distribution around the world (Rosenman, 1991a, Jenkins, 1988).

The hypothesis that certain individual characteristics made a person more vulnerable to developing CHD - and more specifically coronary heart disease (CHD) emerged from clinical observations reported by Friedman and Rosenman (Friedman \& Rosenman, 1959). They proposed that there was a pattern of behavior common to most of their patients that was not as frequently observed in individuals without CHD. That behavior style was labelled the Type A Behavior Pattern (TABP) and included such personal attributes as impatience, competitiveness, high job involvement, fast and emphatic speech, brisk gestures, and hostility and anger in communication with others (Jenkins, 1988, Rosenman, 1990). TABP characteristics are most easily observed in the work environment where challenges and stressful situations provide ample opportunities for Type As to display their particular behavioral style (Renault de Moraes, Swan, \& Cooper, 1993 , Denollet \& De Potter, 1992).

The possible pathways that emotional and behavioral factors may follow to produce organic damage have been widely investigated. In the case of TABP and CHD, one of the most extensively studied hypotheses involves the hyperreactivity of the cardiovascular system in Type As when facing specific stimuli. It has been proposed that, in the presence of stressful stimuli, Type As show an increase in various indicators of cardiovascular activity, such as blood pressure (BP), heart rate (HR) (Dembroski \& McDougall, 1983), vasodilatation in skeletal musculature in the forearm and an increase of plasma norepinephrine (Williams, Lane, Kunh, Melosh, White \& Schanberg, 1982). BP variability has been found to increase in Type As when they carry out challenging cognitive tasks (Elliot, 1988, Contrada \& Krantz, 1988). Both, young and adult men, exhibit cardovascular hyperreactivity when performing arithmetic tasks (Williams, Suarez \& Kunh, 1991). In contrast, Type Bs have been shown to exhibit hyperreactivity of the alphaadrenergic/vagal response when faced with stressful stimuli, such as the cold-pressor test, 
which may explain their lower cardiovascular risk in relation to Type As (Muranaka, Lane, Suarez, Anderson, Suzuki \& Williams, 1988).

It is believed that this exaggerated response of Type A individuals when maintained over time and in daily activities, could result in structural damage to the coronary arteries by increasing plasma cathecolamines which could then lead to atherome formation and a subsequent narrowing of the arterial lumen (Abbot \& Sutherland, 1991). It has also been proposed that persistent hyperreactivity progressively leads to elevated $\mathrm{BP}$ at rest (Warwick-Evans, 1990).

An extensive body of literature exists that provides evidence of the link between hyperreactivity and cardiac illness, however, this discussion goes beyond the scope of this study. Suffice it to say that the findings are ambiguous and that disagreements point to the need for further research (Rosenman, 1991b, Krantz \& Manuck, 1984).

This study was designed to assess TABP, health habits, and biological risk factors for coronary heart disease in a group of high ranking managers. Given the privileged occupational level of these employees it was considered interesting to evaluate their medical and life style risk factors. The second purpose of this study was to register cardiovascular responses, HR and BP, in the work environment during two challenging cognitive tasks. Second, cardiovascular reactivity was assessed with an eye to establishing a possible link between Type A behavior and indicators of CHD, and to Type A Type B differences in reactivity. In addition, subjective reactivity (tension scale) was also evaluated.

\subsection{METHOD}

\section{Participants}

Sixty-two top managers (president, board of directors, and high-ranking managers) of a very prestigious oil company in Caracas wee invited to participate in the study. Ten refused to, nine due to time constrains and one on principle. The final group, therefore, consisted of 52 subjects (46 men and 6 women). Their mean age was 50 years (range: $38-59$ years). Someone from the corporation medical department informed all participants about the purposes of the study. 


\section{Variables and instruments}

1. Type A Behavior Pattern. The Structured Interview (SI) for TABP was used (Chesney, Eagleston \& Rosenman, 1980). The interview was carried out by trained researchers. Appointments were arranged by telephone with each participant and the SI was carried out in their private offices. It had a duration of approximately 25 minutes and it was recorded for its later analysis. The original script was used, plus one or two additional spontaneous questions to encourage Type A behaviors. At the end of the SI, the interviewer made a first evaluation. Later on, after having listened to the recording, he made a final evaluation. A second expert also listened to each recording and provided an independent evaluation. The reliability between the interviewer and the second expert was 94.44. Participants were classified as Type A1, Type A2, Type X or Type B.

2. Health habits and biological risk factors. Data on alcohol consumption, smoking habits, exercise, and obesity were obtained from participants by self-report. A personal history of cholesterol level, tryglicerides and blood pressure was obtained from the participant's medical record.

3. Psychophysiological reactivity. $H R, D B P$, and SBP were registered using digital recording equipment (Dinamap). Participants performed two cognitive tasks for the evaluation of cardiovascular reactivity. The first one was an abstract reasoning task, the Raven Progressive Matrices (RA), and the second one was a mental arithmetic task that involved regressive counting (ARI). During the RA, seven matrices of the highest level of difficulty were presented for 45 seconds on a slide projector after the presentation of two much easier practice matrices. Participants responded verbally. For the ARI participants had to count down from 3283 by 7 until they were told to stop.

\section{Procedure}

All reactivity tests were always carried out in the morning. Upon arrival an initial BP and HR measurement was taken. Then, the participants were told how to respond to the tension scale (TS) (from $1=$ as calm as possible to $10=$ as nervous as possible) when it was required from them throughout the session. Some brief relaxation instructions were then given, and the experimenter left the room for 4 minutes, after which a base-line recording was made automatically. The experimenter retumed to the room and instructions for the first task were given. Some challenging remarks such as that. responses should be given aloud in the minimum possible time, that he expected an optimal performance, and that other managers 
had done very well previously, were added. Response time was measured with a chronometer. Between slides 2 and 3 , and 6 and 7, of the abstract reasoning tasik, HR, SBP, and DBP were recorded. At the end of the task participants responded again to the TS.

After a 3-minute resting period and another recording of the cardiovascular indicators, the ARI task was presented. Participants were asked to give their answers aloud on two occasions, one minute apart. After each response, another recording was done.

At the end of both tasks and after listening to the participant's comments, HR, BP, and TS were again recorded, just before the participants had to leave. Thus, the experimental session consisted of six phases, namely:

Phase 1: Arrival: SBP1, DBPI, HR1, TS!

Phase 2: Baseline SBP2, DBP2, HR2, TS2

Phase 3: RA: SBP3, DBP3, HR3, TS3

Phase 4: Between tasks SBP4, DBP4, HR4, TS4

Phase 5: ARI: SBP5, DBP5, HR5, TS5

Phase 6: Out: SBP6, DBP6, HR6, TS6.

It is important to note that no instructions to refrain from coffee or smoking were given. Nor was room humidity or temperature controlled as is common in laboratory psychophysiological research. The aim was to obtain reactivity responses in natural conditions.

\subsection{RESULTS}

The group is first described in terms of proportions of health indicators and Type A. Performance levels are then described in terms of time of responding and accuracy of responses. This is followed by the analysis of absolute physiological measures, peak values, and reactivity responses. In all cases, Type A-Type B differences are established.

\section{Biological risk factors and health habits}

The most frequent risk factor found in this group was a family history of candiovascular problems $(78 \%)$, followed by a high cholesterol level (40\%), hypertension $(39 \%)$, a personal history of cardiac problems $(35 \%)$, and high triglycerides $(35 \%)$. Figure 2.1. shows percentages of participants with $0,1,2,3,4$, and 5 biological risk factors. It is worth noting that only $8 \%$ had none of the risk factors evaluated. 
Figure 2.1. Percentage of participants with $=0,1,2,3,4$, and 5 biological risk factors (high cholesterol level, high triglycerides level, high blood pressure, family history and personal CHD history). RF= Risk Factors

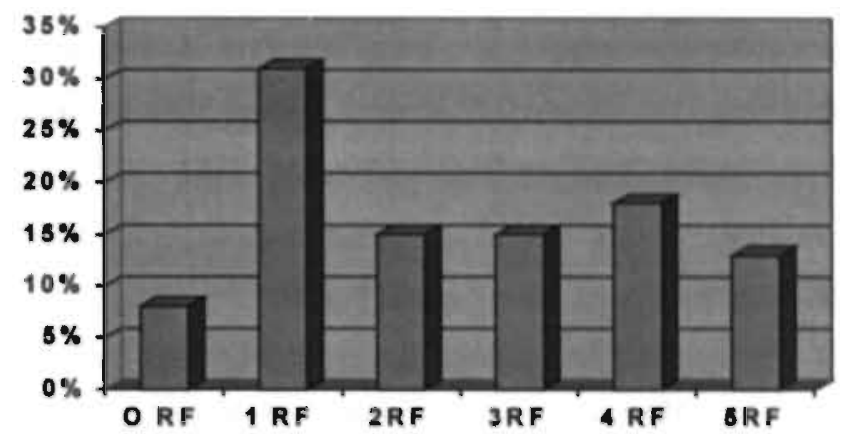

High alcohol consumption (more than three drinks a day) was the most. frequent inadequate health habit associated with CHD (51\%), followed by sedentariness (46\%), smoking (28\%) and, lastly, obesity (18\%). Figure 2.2. depicts percentages of participants with $0,1,2,3$, and 4 health habits related to cardiovascular risk. The higher percentage was for those with two risky health habits.

Figure 2.2. Percentage of participants with $=0,1,2,3$, and 4 risk habits (high alcohol consumption, smoking, overweight, and sedentariness). $\mathrm{RH}=\mathrm{Risk}$ Habits

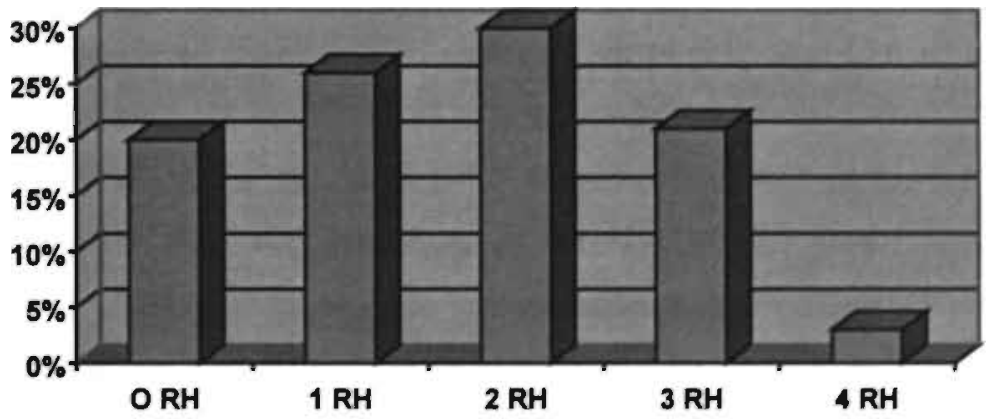




\section{Type A Behavior Pattern}

Results showed that $44 \%$ of the participants were evaluated as Type Al and $41 \%$ as Type A2. This was followed by a much lower percentage: of 'Type X $(9 \%)$ and Type Bs (6\%). When A1s and A2s were grouped together, it was found that Type As had experienced more previous cardiac problems than Type Bs $(\mathrm{p}=, 039$; Kendall's Tau=. 208). In addition, Type As reported having more hypertension than Type $\mathrm{Bs}(\mathrm{p}=, 030 ; \mathrm{tau}=, 225)$.

\section{Performance in reactivity tasks}

There were no gender differences in performance on the two tasks. Therefore, results are shown for all participants together in Table 2.1. As can be seen, there were few correct responses on the ARI task. When looking at the relationship between time of response and accuracy, it was found that they correlated positively ( $r=.25, p=.027$ ) meaning that participants who took longer to respond gave more correct answers.

Table 2.1. Descriptive measures of performance in the abstract reasoning task

\begin{tabular}{lccc}
\hline & X (SD) & Observed range & $\begin{array}{c}\text { Possible } \\
\text { range }\end{array}$ \\
\hline Time of responding (sec.) & $25.46(7.62)$ & $0.9-45$ & $0-45$ \\
Total number of responses & $5.98(1.23)$ & $3-7$ & $0-7$ \\
Number of correct responses & $1.21(1.04)$ & $0-3$ & $0-7$ \\
\hline
\end{tabular}

In the ARI task, $51 \%$ of the responses of participants were correct with a mean number of arithmetic operations (subtractions) of $16.15(\mathrm{SD}=14.25)$. Forty-nine percent of the responses of the group were incorrect (mean=19.11;SD=8.10).

There were no differences between Type As and Type Bs in performance levels, for either response time or accuracy.

\section{Cardiovascular variables and tension scale}

Preliminary analysis showed that there were gender differences in SBP and DBP with men showing higher values. Consequently, data from males and females could not be 
analyzed together. It was decided not to include data from women as there was only six of them. Due to this small number, no statistical analyses were performed with the female data, limiting interpretation to a qualitative analysis that will be presented later. The cardiovascular data analysis was therefore based on the 46 male participants.

The TS, SBP, DBP, and HR measures were compared across the six evaluation phases using one-way ANOVA. When significant differences were found, multiple comparisons were carried out by using the modified least significant differences (ModLSD) test to account for the various comparisons at that significant level.

There were significant differences in the TS responses for the six experimental phases $[\mathrm{f}(4215)=12.63 ; \mathrm{p}=, 001)]$. Tension levels on arrival and during performance were significantly higher than those during the resting and final periods. There were no Type AType B differences in subjective tension (Table 2.2.).

Table 2. 2. Mean values for the subjective tension scale y cardiovascular measures during each phase of the experimental session

\begin{tabular}{lccccccc} 
& Arrival & Baseline & AR & $\begin{array}{c}\text { Rest- } \\
\text { period }\end{array}$ & MA & $\begin{array}{c}\text { End- } \\
\text { session }\end{array}$ \\
\hline TS Range: $0-10$ & $4.7 b$ & $3.2 a$ & $5.0 \mathrm{~b}$ & NE & $5.3 b$ & $3.4 b$ \\
SBP (mmHg) & $145.1 b$ & $130.9 a$ & $152.0 b c$ & $136.9 a$ & $153.5 b c$ & $138.7 a$ \\
DBP (mmHg) & $84.7 a$ & $79.8 a$ & $91.0 b$ & $82.4 a$ & $90.1 b$ & $84.1 a$ \\
HR (bpm) & $66.8 a$ & $66.1 a$ & $74.9 b$ & $66.1 a$ & $77.4 b$ & $65.6 a$ \\
\hline
\end{tabular}

Different letters indicate significant differences. $p<.05$

S: Tension scale

BP: Systolic Blood Pressure

DBP: Diastolic Blood Pressure

HR Heart Rate (beats per minute)

NE: Not Evaluated

The three cardiovascular indicators also showed significant differences during the experimental phases [SBP: $F(5.257)=7.78 ; p=.001 ; D B P: F(5.258)=6.15 ; p=.001 ;$ HR: $F(5.252)=9.49: p=.001]$. SBP showed the highest values during the performance of tasks, Also, SBP upon arrival was significantly different from the measures during the rest period and at the end of the session. DBP and HR during tasks were significantly higher on arrival, during the rest period, and at the end of session measures. Thus, the two experimental tasks, were effective in producing cardiovascular activation in this group as well as on the TS. 
Moreover, SBP reflected the activation in participants upon arrival at the session prior to the baseline measurement.

In order to take into account differences in baseline levels, cardiovascular reactivity was calculated by using the following formula:

\section{Phase measure - Rest measure}

\section{100}

Rest measure

FIgure 3. Porcentage of participante with $m$ aximum reactivity in usch phase of the session.

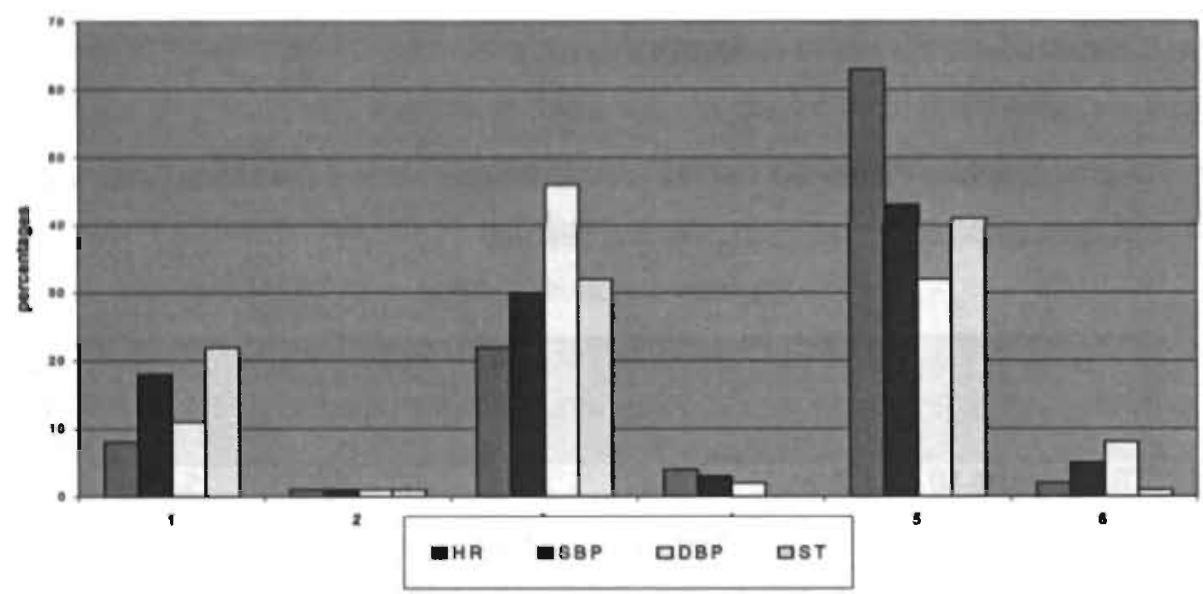

$1=$ Arrival

2=Base-line

$3=$ Abstract Reasoning (AR) task

4=Rest Period

$5=$ Mental Arithmetic (MA) task

$6=$ End-session

Maximum reactivity values were also obtained by calculating the greatest change between the resting phase and each of the other experimental phases. Similarly, in the case of the TS, maximum tension change was calculated by subtracting the baseline measure from the greatest tension evaluation throughout the entire session.

Maximum reactivity in each phase is shown in Figure 3 . The majority of participants had a greater reactivity during the ARI task. This was the case for HR, SBP and for the TS. It is interesting to note that the maximum DBP' response occurred during the RA. 


\section{Cardiovascular response and performance levels}

Multiple Regression analyses carried out with performance level as the variable to be predicted showed that the HR in Phase $\mathbf{1}$ (arrival) was negatively related to time taken to respond to the tasks $(\beta=-415, \mathrm{R}=172, \mathrm{p}<.005)$. In other words, the greater the HR activation was upon arrival, the less time it took the participants to respond.

In the ARI task, HR and DBP in phase 6 (just before leaving the room) were positively related to the number of correct answers given $((\beta=-317, R=.078, p<.04 ;,(\beta=$. $.490, \mathrm{R}=.240, \mathrm{p}<.001$, respectively).

\section{Type A-Type B differences in cardiovaseular variables}

In spite of the reduced number of Type B participants, one-way ANOVA were tentatively carried out to assess Type A-Type B cardiovascular differences. Results showed that they differed only in the measure of SBP taken at the end of the experimental session. Type As had a higher response than Type Bs [F (1,33); $\mathrm{p}=0263)$.

\subsection{DISCUSSION}

Descriptive analyses showed that in this group of managers there were high levels of biological risk factors and inadequate health habits that are associated with cardiovascular diseases. Of particular interest is the high percentage of Type As in this occupational group $(85 \%)$. The proportion of participants with more than two risk factors was also elevated. Moreover, only $8 \%$ had only one biological risk factor, whereas $82 \%$ had one or more. In the case: of health habits related to cardiovascular diseases, $20 \%$ had none compared to $80 \%$ who had one or more. In summary, in spite of their high occupational level, this group seemed to have a high cardiovascular risk.

Murza (1990) argues that managers have a healthy life style, that they feel healthier than members of other occupational groups, and that their high socioeconomic level is associated with a better quality of life. Nevertheless, this high socioeconomic level might bring with it a number of inadequate health habits. Moreover, in men with a mean age of 50 years, high number of risk factors is a matter of concern. 
The high percentage of Type As reinforces the notion that individuals with high competitiveness, time pressure, and hostile behaviours are generally those who will attain high occupational levels within a large corporation. This behavioral pattern is often beneficial for organizational development and is thus reinforced in its employees (Margolis, McLeroy, Runyan, \& Kaplan, 1983).

In Venezuela, as part of a larger research project, two studies that evaluate Type A behavior in different groups have recently been completed. In both studies the SI was used. In the first study (Micale, 1992), 90 female middle level managers and clerks were evaluated and $53.3 \%$ were found to be Type As. In the second study (Yañes, 1992), 98 policemen were evaluated $34.69 \%$ of whom result turned out to be Type As. The groups are obviously very different and the results show the importance of such variables as sex and occupation in Type A behavior.

The data regarding the small number of female participants in this study can only be subject to a brief, qualitative interpretation. They were six women who held similar high-management positions comparable to those of the male participants. Five of them were evaluated as Type As. The sixth woman, who was assessed as Type B, was the manager in charge of the library and publications of the company, a job that does not call easily for Type A characteristics. Moreover, this woman was only one who was married and had four children. The other five participants were single and childless, indicating perhaps their high job involvement. Future studies in female groups are needed to determine the relative importance of job versus family, especially the perception of family, as an obstacle to attaining high positions in large corporations. Previous findings on the relationships between psychosocial variables and CHD in women underline this need for future studies (Graff-Low \& Fleisher, 1994).

\section{Cardiovascular reactivity}

Cardiovascular reactivity results seem to indicate that there was response specificity in the various phases of the experimental session. In particular, initial activation of the SBP was probably a reflection of what the participant thought was expected of him. In previous studies it has been pointed out that reactivity is a multi-component construct and that different tasks or situations may result in distinct patterns of physiological response (Krantz \& Manuck, 1984). Our results support this notion and add to the importance of the initial, individual response prior to the rest period before the baseline recording. 
The initial HR measurement was also of interest in this study, but this time related to performance levels. HR at the beginning of the session was related to the time of response in the AR task. As the time of responding was negatively related to the number of correct responses, it could be argued that the initial HR activation was unfavourable to a good performance on that first task. Moreover, final HR was related to performance on the ARI task emphasizing the relevance of initial and final cardiovascular reactivity. Both measures may be more relevant physiological response indicators than the resting and task levels that are usually reported in the literature.

The higher SBP response found in Type As as compared to Type Bs at the end of the session further points to the relevance of those measures. Although there were no differences in their cardiovascular responses during the various rest and task phases, the preoccupation of Type As with their performance level or their inability to recover a baseline level was most likely responsible for their higher response.

In line with our findings it has previously been reported that Type As do not differ from Type Bs in their cardiovascular response when performing an arithmetic task (Muranaka, et al., 1988). In those studies, results also showed that Type Bs were more reactive than Type As when responding to a cold stimulus (alpha-adrenergic). Authors conclude that those results support the hypothesis that Type As and Type Bs differ in their autonomic nervous system balance with Type As being more prone to a higher betaadrenergic response (like the one that is common in a mental arithmetic task), while Type Bs are more predisposed to an alpha-adrenergic reaction.

In a review of 45 studies, (Myrtec \& Green, 1984)) it was found that the only cardiovascular indicator that differed between Type As and Type Bs was SBP reactivity. Dembroski \& McDougall (1983) also found similar results with SBP and HR. Cinciripini (1986), on the other hand, reported a review of 14 studies, 10 of which showed that Type As were more reactive than Type Bs. He points out that controversial findings may be due to different Type A classification methods or different tasks, or to the fact that only a subgroup of Type As are more reactive. Our results support the SBP difference between Type As and Type Bs, but only in the final measure recorded.

The proportion of Type Bs was much smaller than that of Type As and this limits the scope of possible interpretations and conclusions that can be drawn from this study. Nevertheless, with a conservative methodology and statistical analyses, that take into account the sample size, we were able to obtain significant results.

In summary, the hypothesis of a greater reactivity of Type As during the performance of a cognitive task in semi-natural conditions was not supported in this study. 
From our finding it may be concluded that reactivity is not part of the pathogenic process involved in the coronary disease to which Type As are most prone. Nevertheless, the findings in relation to SBP at the end of the session should encourage further research into the cardiovascular responses of Type As and Type Bs. Rather than observing then while performing mental tasks or undergoing stressful daily hassles, it may be wise to do so during waiting periods, at moments of high expectations, or once stressful events or tasks have been finished. 


\section{CHAPTER 3}

\section{DIFFERENCES BETWEEN INFORMANTS ABOUT TYPE A, ANGER, AND SOCIAL SUPPORT AND THEIR RELATIONSHIP WITH BLOOD PRESSURE}

\subsection{SUMMARY}

In a study on the influence of different environments on self-perception and that of significant others, behavioral manifestations of Type A, Anger/Hostility, and Social Support were assessed in their natural environments. It was hypothesized that due to different environmental demands, participants will be perceived as displaying differences in Type A behavior, anger/hostility, and social support at home and at work. Participants were 45 university employees, constituting a convenience sample. Assesșment included Type A Stractured Interview scores of participants and questionnaire scores for Type A, anger/hostility and social support of participants, spouse and work supervisor. Other risk factors for coronary artery disease (i.e., blood pressure, smoking, previous heart condition, being sedentary, and family history), and intensity and frequency of stress situations, and their relationships with Type A, anger/hostility, and social support were also examined.

Participants and spouses agreed significantly on all measures (i.e., Type A, anger/hostility, and social support), whereas participant and work supervisor agreed only on Type A behavior. Spouse's and work supervisor's evaluations of the participant's behavior were not associated. Multiple regression analysis showed that the level of disagreement between the different informants with respect to Type $\mathrm{A}$ and social support was associated with elevated blood pressure. These results suggest that the perception of Type A behavior, anger/hostility, and social support may be situationally determined.

2 Bagés, N., Warwick-Evans, L., \& Falger, P.R.J. (1996), Differences between infoormants about Type A, anger-expression, and social support and the relationship with blood pressure. Psychology and Health, 12, 453-465. 


\subsection{INTRODUCTION}

After more than 30 years, the Type A concept is still alive although it has undergone severe criticism due to controversial results that question its validity as a general risk factor for coronary heart disease (CHD) (Dembroski \& Costa, 1987; Williams \& Barefoot, 1988; Williams, Suarez, Kuhn, Zimmerman \& Schanberg, 1991) and its evaluation procedures (Eysenck, 1991). Type A behavior pattern (TABP) and its separate components are nowadays a rich area of study that continues to deserve further attention.

The evaluation of TABP and its components is a major issue in coronary-prone behavior research. This is due to findings that different TABP assessment procedures (i.e., interviews and questionnaires) resulted in different predictive power of CHD (e.g., Booth-Kewley \& Friedman, 1987; Matthews, 1982). Traditionally, Type A has been assessed by using interviews, in particular the Structured Interview (SI) (e.g. Rosenman, Swan \& Carmelli, 1988) and more recently the Videotaped Structured or Clinical Interview (VSI) (Friedman \& Powel, 1984). The former two were developed to explicitly assess overt behavioral manifestations of Type A.

Studying individual behavior in natural environments in relation to Type A - Type B manifestations has been highly neglected. The assessment based on observations of TABP during daily activities could improve its predictive power and the understanding of the nature of Type A behavior (Matthews, 1982). Comparisons of observational with self-report data seem most suitable to clarify both conceptual and evaluation issues (Swan, Carmelli \& Rosenman, 1990).

By evaluating the overt behaviors particular to Type A and its components in daily life, valuable information to that provided by the SI would be added. Due to the brevity of this interview that is limited to about 15 minutes of observation under specific conditions, it may fail to elicit the full range of Type A behavior, perhaps due to interview style, participant's attention, or not tapping particular aspects that would prompt behavioral components of Type A to emerge. By contrast, prolonged observation during daily life would provide a more representative sample of an individual's behavior and also add more to the natural history of the behavior and coping style of the individual (Friedman, 1992). The lack of insight about their own coping strategies that has been attributed to Type As, and the fact that one's behavior may change according to the individual's perception of different environmental 
demands (Bennett, Gallacher \& Johnston, 1990), therefore varying the probability of observing Type A behaviors in dissimilar surroundings, such as home and the working place, also makes observation in daily life most relevant.

Moreover, by assessing presence of Type A in different environmental settings, the state vs. trait issue can also be addressed. In general, one is classified as either Type A or Type $B$ as if these were static personality traits rather than considering the possibility that situational or environmental variables may have an effect on the quantity or quality of display of those behavior patterns. Conceptually, Type As overreact when encountering certain situations that pose a threat or challenge to self-esteem or involve difficult problem solving (Smith, 1989); therefore, it is considered an environmentally provoked syndrome (Suls \& Sanders, 1989). However, specification of the environments in which these challenges may occur is not included in those conceptualizations. In the present study, a transactional model will be tested by observing differences in Type A behavior and its components (e.g., anger manifestation), at home and at work. It is thought that the same type of stressor, under different environmental demands, will pose a different challenge and, therefore, evoke different behavioral, and perhaps also physiological, responses.

With specific regard to anger, Chesney (1985) strongly suggests that assessment should take place by observing the individual in the natural environment, although the difficulties involved, namely, the possible influence of the observer and the costs in terms of personnel, decoding and analysing the data, are acknowledged. Obviously, it is nol always feasible for an experimenter to observe individuals in their natural environments. Therefore, others close to the subject such as spouse or partner or colleagues and supervisors at work may provide important information regarding specific behaviors, such as Type A and its components, and aspects related to social support in the subject's daily life. It may be argued that these "natural" observers are untrained and, therefore, there would be no control about the accuracy of observations. Whereas this may seem a valid argument, the advantages of this method seem to outweigh its disadvantages. Eliminating the "experimenter" as much as possible is one of the aims of social psychology in order to diminish expectancy effects (Silverman, 1977). Asking others in the real world to provide information about the participant is a way of attaining this.

While conducting the SI it is thought that Type A emerges as in real life, but in some instances it may fail to do so. Naturalistic, unobtrusive observation may contribute to a more realistic picture of the person's behavior pattern. The untrained spouse and work supervisor have the advantage of being able to observe the subject unobtrusively and they have done so for a long period of time; their evaluations are not restricted to about 15 minutes of 
observation. Most importantly, there is the opportunity to compare their information with both the SI and self-report measures. Actually, these reporters are not even aware that they are observers until they reply to questions about the participant. There is not even a question of being asked to observe first and to reply to a questionnaire a few days later, which would bring about the problem of expectancy. Moreover, the relevance of studying anger expression in a natural environment is evident from studies where only the overt manifestations of anger, and not their experience or repression, were related to hyperreactivity and CHD (Siegman, 1992).

\section{Type A, Anger, and Social Support}

It has been repeatedly pointed out that Type $\mathbf{A}$ is usually measured in isolation, without giving much importance to other behavioral modulators that may interact with it (Matthews (1983), and that behavior patterns should be observed in their psychosocial contexts (Orth-Gomer \& Undén, 1990). Social support, defined as the individual's perception of available sources of support, has been found to moderate the risk associated with Type A most strongly (Blumenthal, et al., 1987; Orth-Gomer et al., 1990). Recent research shows contradictory results in the relationship between hostility and social support (Houston \& Vavak, 1991; Smith \& Houston, 1987). Measuring social support in a more extensive manner while focusing on the behaviors that are supportive or support seeking (e.g., social support from family and work resources and observing relationships with self-reported and observed anger), would add valuable information. The way in which others perceive how an individual interacts socially, in contrast to the subject's opinion about social support, and how this relates to health may also provide valuable information about the moderating effects of social support upon health and CHD.

To summarize, the main objectives of the present study are:

To explore relationships and levels of agreement between self-report measures and those of spouses and work supervisors about Type A, anger expression, and social support; 2. To examine relationships between the different assessment procedures and levels of agreement between them with other CHD risk factors; 3 . To investigate methods of evaluating overt behaviors involved in Type A and anger expression. 


\subsection{METHOD}

\section{Participants}

All participants came from the University of Southampton community and were recruited by posting signs on campus and announcements in university papers. All subjects of 35 years and over wanting "to receive information about their heart risks.." were addressed. Forty-five participants responded: 17 males and 28 females between 34 and 62 years of age. They consisted of 18 secretarial staff, 8 catering-, security-, and maintenance-personnel, 7 department managers, 6 technicians, and 3 lecturers and 3 older students. Data on Type A behavior, anger expression, and social support were also collected from spouses and work supervisors. As some participants did not have a spouse or partner and the students did not have a work supervisor, full data were available on 20 participants, whereas 12 only had supervisor's data, 6 only had spouse data, and 7 had no data from either spouse or supervisor.

\section{Instruments}

In order to evaluate the behavioral manifestations of Type $A$ and anger in a comprehensive manner, the following procedures were used:

1. Structured Interview. The Structured Interview Protocol (Chesney, Eagleston \& Rosenman, 1981) takes about 20 minutes to conduct. All interviews were carried out by the first author, who had been trained by Dr. Rosenman, and were tape-recorded for later analysis. A first assessment was performed by the interviewier at the end of each interview and a second scoring was carried out on the basis of the recordings. Participants were classified as either Type A1, A2, X, or B3. A random sample of the tapes was co-rated by another trained interviewer. Inter-rater reliability between rater and co-rater was 85 .

2. Personal Relations Chestionnaire (Social Support/Anger/TABP Questionnaire). This questionnaire is an adaptation of three scales and was developed specifically for this study and consists of three adapted scales. Items 1 to 22 were taken from the Social Support Questionnaire (Dunn, Putallaz, Sheppard \& Lindstrom, 1987), which is a longer version of a scale originally developed by Fleming, Baum, Gisriel and Gatchel (1982). Only the questions with respect to observable social support behaviors were included here. Items 23 to 31 were the anger items from the Self-Concept Scale or Anger-Hostility Complex Questionnaire (McDermott, 1990). Again, only those questions about overt expressions of anger were included. Items 32 to 41 were a written version of those 
questions in the SI that refer to behavioral manifestations of Type A. The reliability of each of the scales was calculated separately; Cronbach's alphas were: 0.75 (Social Support scale; 21 items); 0.82 (Anger/Hostility scale; 10 items); and 0.62 (Type A scale; 9 items), respectively.

3. Social Support/Anger/TABP Questionnaire for Spouse/Partner. This questionnaire's contents were identical to the one described above, except that the pronouns all refer to the participant. The spouse/partner was asked to provide answers referring to the overt behaviors of the partner. It was sent through the participant in a sealed envelope with the explicit instruction not to "collaborate" with the spouse in answering it.

4. Social Support/Anger/TABP Questionnaire for Work Supervisor. This was a short version of the questionnaires described above. It contained 4 items on social support, 5 items on anger, and 5 items on Type A. Only items considered to be observed most likely in the workplace were included. These were identical to some of the items in the other two scales. By using a short version it was thought that the return rate would be increased. This questionnaire was also sent through the participant.

5. Sociodemographic and Health Questionnaire. This questionnaire included the following sociodemographic items: Type of work, time working in the university (mean number of years), marital status, and educational level. Participants also reported on their cardiovascular health history (i.e., hypertension, angina and MI) based on their physicians' communications. In addition, smoking, physical exercise, family history of CHD, and sleep characteristics (hours and problems) were assessed through this questionnaire.

6. Blood Pressure. Blood pressure (BP) was recorded before, during and after the SI. The BPcuff was placed on the left arm and a random zero sphygmometer was employed as protection against observer bias in clustering the BP-measurements. The interview was unexpectedly and momentarily interrupted after question \# 12, when a second BP reading was recorded, after which the interview was resumed.

\section{Procedure}

After permission from the ethical committee to carry out the study, posters soliciting volunteers to participate in a study about CHD risk factors were disseminated throughout the university. Volunteers attended the experimental session according to their convenience so that their work routine would be minimally interfered with. After anival, they were led into a specially prepared room that included the BP-equipment and a tape-recorder, the procedure was explained, and informed consent was obtained. All subjects agreed to participate. The 
time between arriving and the first BP recording was approximately 10 minutes and was considered a rest period in which the participants remained seated. Participants were then told about the SI which started immediately after. About ten minutes after the SI hadl begun it was interrupted momentarily at question 12 and a second $\mathrm{BP}$ reading was recorded. Participants were not previously informed about this. The last reading was immediately after the interview was, finished. Then, after instructions, the participants began cornpleting the questionnaires and were left on their own to do so. Meanwhile, the first assessment of the Type A interview was carried out in an adjoining office in order to provide participants with their scores, together with their BP readings.

The spouse and work supervisor questionnaires were then handed out in sealed envelopes with a return address. Participants were informed what type of questionnaires these were and were asked to give these, unopened, to the spouse and work supervisor and not to make any comments about the contents previously to answering and returning the questionnaires through the University mail system once completed. No one refused to do so.

\subsection{RESULTS}

\section{Statistical Analysis}

The participants are first described in terns of sociodemographic and health status. Relationships of Type A behavior, anger/hostility, and social support within and between each of the informant's questionnaires will be analyzed using Pearson product-moment correlation coefficients (Hays, 1974). Since BP was recorded three times, maximum and minimum systolic and diastolic BP (MAXSBP, MINSBP, MAXDBP, and MINDBP) were analysed.

Levels of agreement between informants' and participants" BP measures were determined by subtracting the total score on each of the participants' questionnaires from the scores of the corresponding spouses' and supervisors' guestionnaires. This means that participants with a negative level of agreement had a low self-perception of Type A or social support and those with a positive level of agreement a higher self-perception on those variables than their corresponding counterparts. Levels of disagreement were analyzed by means of multiple regression analysis. 


\section{Sociodemographic and health characteristics}

Of all participants, $76 \%$ was married, $4 \%$ was single, and $20 \%$ was divorced. Thirty-one percent of all participants had attended only primary and high school, $41 \%$ reported a completed higher vocational training, and $19 \%$ had completed a university education. Descriptive statistics for health variables are reported in Table 3.1. Although these were self-reported data, and, therefore, should be interpreted with some caution, it shows that this sample was generally in good health, in particular with respect to exercise. Prevalence of past illness, which may be associated with CHD, appeared to be relatively low, although it did not appear possible to accurately check this against current UK health statistics.

Table 3.1. Descriptive Statistics About Health and Health Habits

\begin{tabular}{ll}
\hline Variables & $\%$ Yes \\
\hline Hypertension (self-reported) & 15.6 \\
Hypotension (self-reported) & 4.4 \\
Myocardial Infarction & 2.2 \\
Angina & 11.1 \\
Bronchitis & 13.3 \\
Ulcer & 4.4 \\
Asthma & 11.4 \\
Regular Exercise (>1/week) & 79.5 \\
Current Smoking & 2.3 \\
Past Smoking & 34.9 \\
Passive Smoker & 40.9 \\
\hline
\end{tabular}

Evaluation of Type A behavior.

According to the SI, $60 \%$ of the participants were assessed as Type As (including A1 and A2) and $40 \%$ as Type Bs (including Xs and B3). Participants rated as Type As had higher scores on the self-reported Type A scale than Type Bs $(t=2,14 ; p=039)$, meaning that the self-report scales discriminated well between Type As and Type Bs.

Means and SD for self-reported Type A, anger, and social support, and the corresponding spouse and supervisor ratings are shown in Table 3.2. ANOVA with respect to 
the means for the three informants and the three variables showed significant differences in social support $[\mathrm{F}(2,96)=22,73 ; \mathrm{p}<.0001$ ); post-hoc comparisons (Bonferroni-tests) showed differences between the supervisor scores and those of the other two informants.

Table 3.2. Means and Standard Deviations of Type A Behavior, Anger Expression, and Social Support for the three informants.

\begin{tabular}{|c|c|c|c|c|c|}
\hline & \multicolumn{2}{|c|}{ Type A } & \multicolumn{2}{|c|}{ Anger } & Social Support \\
\hline & Mean (SD) & $\operatorname{Min}-\operatorname{Max}$ & $\operatorname{Mean}(S D)$ & Min-Max & Mean (SD) Min-Max \\
\hline Participant & 1.35 .51 & $.33-2.78$ & .67 .56 & $00-2.22$ & $1.81 .38 \quad .05-2.73$ \\
\hline Spouse & 1.31 .48 & $.11-2.33$ & .58 .58 & $00-2.33$ & 1.81 .40 \\
\hline $\begin{array}{l}\text { Work- } \\
\text { supervisor }\end{array}$ & 1.55 .44 & $.80-2.40$ & 57.85 & $00-3.00$ & $2.45^{*} .55 \quad .80-3.00$ \\
\hline
\end{tabular}

* Social Support significantly higher $(\mathrm{p}<01)$.

Correlations within and between scales (participant, spouse, and work supervisor).

Table 3.3. shows the correlations within each of the questionnaires completed by the three informants: participant, spouse, and work supervisor. Although informants number differed, there were significant positive relationship between TABP and anger in all three evaluations and a significant negative correlation between anger and social support in the work supervisor questionnaire.

Relationships between participant, spouse, and work supervisor on the three variables are shown in Table 3.4. Participant and spouse scores were positively associated for all three variables, whereas participant and work supervisor only agreed with respect to Type A behavior. Interestingly, spouse and work supervisor only agreed in their Type A and anger perception on the participant and then at a marginal significance level. 
Table 3.3. Correlations Between Type A Behavior, Anger Expression, and Social Support for the Three Informants.

\begin{tabular}{|c|c|c|c|}
\hline $\begin{array}{l}\text { Informan } \\
\text { t }\end{array}$ & Type A/Anger & $\begin{array}{c}\text { Type } \\
\text { A/Social } \\
\text { Support }\end{array}$ & $\begin{array}{c}\text { Anger/Social } \\
\text { Support }\end{array}$ \\
\hline Participant & $\begin{array}{c}32 \\
(n=45 ; p=.031)\end{array}$ & $\begin{array}{c}.10 \\
(\mathrm{n}=45 ; \mathrm{p}=.512)\end{array}$ & $\begin{array}{c}-.12 \\
(\mathrm{n}=45 ; \mathrm{p}=.425)\end{array}$ \\
\hline Spouse & $\begin{array}{c}.47^{* *} \\
(\mathrm{n}=26 ; \mathrm{p}=.016)\end{array}$ & $\begin{array}{c}.18 \\
(n=22 ; p=420)\end{array}$ & $\begin{array}{c}.028 \\
(\mathrm{n}=22 ; \mathrm{p}=.990)\end{array}$ \\
\hline Supervisor & $\begin{array}{c}.42^{* *} \\
(\mathrm{n}=32 ; \mathrm{p}=0.15)\end{array}$ & $\begin{array}{c}-.01 \\
(\mathrm{n}=32 ; \mathrm{p}=.930)\end{array}$ & $\begin{array}{c}-.49^{* *} \\
(\mathrm{n}=32 ; \mathrm{p}=0.004)\end{array}$ \\
\hline
\end{tabular}

Table 3.4. Zero Order Correlations of Type A Behavior, Anger Expression, and Social Support Between the Three Informants.

\begin{tabular}{|c|c|c|c|}
\hline & Type A & Anger & $\begin{array}{l}\text { Socia! } \\
\text { Support }\end{array}$ \\
\hline Participant/ & $.48^{* *}$ & $.60^{* *}$ & $.57^{* * * *}$ \\
\hline Spouse & $(\mathrm{n}=26 ; \mathrm{p}=0.12)$ & $(\mathrm{n}=26 ; \mathrm{p}=.001)$ & $(\mathrm{n}=22 ; \mathrm{p}=.005)$ \\
\hline Participany & $.42^{* * *}$ & .32 & .32 \\
\hline Supervisor & $(n=32 ; p=016)$ & $(n=32 ; p=.073)$ & $(\mathrm{n}=32 ; \mathrm{p}=.068)$ \\
\hline Spouse/ & .43 & .44 & .47 \\
\hline Supervisor & $(\mathrm{m}-21 ; \mathrm{p}=05)$ & $(n=21 ; p=04)$ & $(\mathrm{n}=17 ; \mathrm{p}=055)$ \\
\hline
\end{tabular}


Comparisons of sociodemographic, psychosocial and health variables.

Earlier, Table 3.1. showed that the present sample was in good health. Few subjects reported the health problems mentioned, and, furthermore, their health habits were adequate. Nevertheless, tests were performed to explore differences with respect to those variables. Those participants who reported ever having been diagnosed as hypertensive, showed higher mean (SD) MAXDEP [89 (11) vs. 79 (9); $\mathrm{p}=, 017$ ], MINSBP [120 (12) vs. 108 (14); $\mathrm{p}=.033$ ], and MINDBP [79 (4) vs. 70 (7); $\mathrm{p}=.003$ ] during the SI session than those who had not. This supports the representativeness of the BP recordings with respect to the self-reported health status of the participants. There were no BP differences between Type As and Type Bs.

Pair-wise comparisons showed that Type As were more likely to have completed higher education (Tau $=-.381 ; p=.01$ ), to have been smokers in the past $($ Tau $=-.295 ; p=$ $.025)$, and to have at least one parent with a history of CHD (Tau $=.293 ; \mathrm{p}=.038)$, than were Type Bs. Moreover, males, were less often rated as Type Bs than were females (Tau $=-.274 ; \mathbf{p}$ $=.034$ ). In addition, those with a dual parental history of CHD had higher scores on anger than those with only one parent with such history (Mann-Whitney $U=18.5 ; Z=-2.47 ; p=$ .047).

Levels of agreement between the informants and blood pressure.

Finally, in order to examine another main issue of the study, the associations of the scores of the three informants and their agreement levels with biological indicators, such as blood pressure, were evaluated. No associations were found between raw scores on each questionnaire and BP. Then, in order to explore level of agreement between participant and spouse, participant and supervisor, and BP, stepwise multiple regression analyses were performed. Table 3.5. shows the standardized B-coefficients, amount of variance explained, and corresponding p-values. Significant asscciations were found between disagreement about social support between participant and supervisor, and elevated MAXDBP, MINSBP, and MINDBP. Also, disagreement about perceived level of TABP between participant and spouse. was associated with elevated MAXDBP. That is, those with lower self-perception of social support and those with lower self-perception of TABP than their spouse or supervisor, and, thus, negative levels, of agreement, had higher BP values. Together, these variables explained $3458 \%$ of BP-yariance, which was highly significant. 
Table 3.5. Stepwise Multiple Regression Analysis: Levels of Agrement between informants and Blood Pressure Measures.

Variables:

MAXDBP

MINDBP MINSBP

$\begin{array}{llllllllll}O \text { of E } & B & \triangle R 2 & O \text { of } & B & \triangle R 2 & \text { Oof } & B & A R 2\end{array}$

Participant's Self-

Report and Superviso

Social Support

Participant's Self-

Report and Spouse:

$1 \quad-.585 \quad .345^{*}$

Type A Behavior

MAXDBP: Maximum Diastolic Blood Pressure; MINDBP: Minimum Diastolic Blood Pressure; MINSBP: Minimum Systolic Blood Pressure; O of E: Order of Entry. ${ }^{*} p<.05$; ${ }^{* *} \mathrm{p}<.01$; ${ }^{* * *}$ $p<.001$.

\subsection{DISCUSSION}

The most important finding from this study is that home and work may represent different psychosocial contexts and that relative stability of emotional responses across these contexts seems to be relevant in relation to possible health consequences. This issue was addressed from a transactional perspective, that is, the increased likelihood of Type As to more frequently create challenges and enduring demands in daily environments than Type Bs would (Smith \& Anderson, 1986); this, in turn, could be physiologically more taxing (Smith, 1989). Moreover, selection of specific challenges and demands from the environment may also depend on the type of environment itself.

Our results show that behavioral responses to a stressful stimulus at home or at work are not identical. In those environmental settings, Type $A$ behavior, or anger expression and social support, are not perceived similarly. The need to further explore this issue seems evident. The transactional model may need to be extended so that the same type of stressors (i.e., challenge, threat to self-esteem or problem- solving capabilities) may pose different demands in different situations and result in different emotional responses and physiological reactions. 
The associations found within each questionnaire showed an important issue with respect to the relationship between Type $A$ and anger expression. In all three instances, there were highly significant correlations between both constructs. Thus, participants themselves, as well as those observing them at home and at work, agreed in that if TABP was present, anger expression was likely to occur as well. Anger and hostility are considered by many authors to constitute the "toxic" components of TABP (Dembroski, MacDoudall, Casta \& Grandits, 1989; Siegman, Anderson, Herbst, Boyle \& Wilkinson, 1991; Smith \& Christensen, 1992). Various, studies in which both components were evaluated found that expression of hostility was highly predictive of CHD, whereas global TABP was not. In the present study, in agreement with the original construct, both anger and TABP were so closely related that they appeared to be part of the same construct. Since this study yielded cross-sectional data ${ }_{5}$ it is. not possible to assess their predictive values separately. Therefore, in future studies, this issue may be evaluated prospectively.

The relationship between social support and anger varied with the environments in which it was evaluated. The significant negative correlation between social support and anger/hostility found at work suggests that, in the work environment, those subjects openly expressing anger are less likely to be perceived as socially integrated. Neither the participants' nor the spouses' questionnaires showed an association with social support, meaning that at home both behaviors are expressed according to the opportunities to do so. The lack of associations between TABP and social support among the three informants leads to the conclusion that Type As may or may not show good social integration or adaptation, depending on the psychosocial contexts in which these variables are evaluated. This could lead to differential health risks as have been found in studies where Type As with good social support had lower CHD risk than Type As with poor social support (Blumenthal, et al., 1987; Räikkönen, Keskiväara \& Keltikangas-Järvinen, 1992).

When examining the relationships and levels of agreement between self-report measures of Type A, anger and social support and those of spouses and work supervisors it was found that, having been asked the same questions about those behaviors, participants and spouses agreed on all measures and whereas work supervisors only agreed with participants on their TABP. On the other hand spouses and supervisors showed no agreement in social support and significant, but low, agreement in Type A and anger perceptions. These disparate observations about the participant at home and at work indicate the actual display of different behaviors according to environmental demands. The possibility that the differences, however, were due to differential characteristics of the observers remains open and deserves further evaluation, perhaps by adding other observers (e.g., relatives or colleagues). 
The second study purpose concerned the interrelationships of the responses from the three informants, the levels of agreement between them, and BP. The mechanisms by which behavior, specifically TABP and anger/hostility, may be related to indicators of cardiovascular disease, or to the protective effects of social support on cardiovascular health, are currently being investigated, and various approaches have been proposed (Orth-Gomér, Rosengren \& Wilhelmsen, 1993; Suls \& Sanders, 1989; Undén, Orth-Gomér \& Elofsson, 1991; Waldstein, Polefrone, Bachen, Muldoon, Kaplan \& Manuck, 1993). These will, however, not be extensively discussed here. There is ample evidence to support the notion that Type As and those displaying anger/hostility are more vulnerable to suffer from CHD (Suls \& Sanders, 1989). One of the pathways that have been proposed is that of cardiovascular hyperresponsivity. Although there are some inconsistencies across studies, the notion that heart rate and BP overshoot when Type As respond to specific situations has been frequently supported (Gallacher, Bennett \& Sharp, 1990; Melamed, Harari \& Green, 1993, Lawler \& Schmied, 1986; Muranaka, et al., 1988).

No single relationships were found between the scores of any of the informants on the psychosocial variables and BP. However, in multivariate analysis, level of disagreement between participants and work supervisors on social support showed a strong relationship with BP. The direction of this relationship was such that if supervisors reported that participants had better support than they themselves thought, MAXDBP, MINDBP, and MINSBP were significantly higher. Therefore, these may results suggest that if someone reports low appreciation of social support compared to what others perceive as such, this appears a good predictor of elevated BP. This may be valuable in terms of intervention procedures for lowering BP, indicating to subjects at risk that resources may be available by increasing awareness of support at work. This may help to reduce an important risk factor such as elevated BP. Type As may in fact be less aware of or less interested in support resources in the environment by primarily trying to mobilize and control their own personal resources. This may sometimes be too demanding or else may ',create inadequate social support resources for themselves..' (Suls \& Sanders, 1989).

Moreover, level of agreement between spouse and participant on TABP showed a negative relationship with MAXDBP, indicating that if spouses thought that participants were more Type A than they themselves did, MAXDBP was higher. This could be explained by the finding that Type As appear to have little insight in their own behavior when a paper-andpencil assessment of TABP is employed, in contrast to the insight obtained my means of the SI-assessment (Rosenman, 1991a). At home, they may display typical TABP manifestations of which they are not aware thenselves, but that the spouse will perceive as such. For 
example, in a study that examined self-report and spouse ratings of hostility, it was found that only spouse-rated hostility contributed to the prediction of CHD (Kneip, Delamater, Ismond, Milford, Salvia \& Schwartz, 1993). Together with our findings, these results suggest that differential observations of overt risk behaviors may be valid indicators of cardiovascular problems.

Finally, the method of evaluating the overt behaviors associated with TABP, expression of anger/hostility, and social support by using different reference sources was found appropriate. Both spouse and supervisor provided information regarding the behavior of the participant in two different environmental settings that could be contrasted with participants' self-reports. Differences in amount and quality of time spent with the subject, as well as differences in type of personal relationships, when comparing spouse and work supervisor reports, are valid issues to be raised when interpreting these results. Spouses are usually involved in a wider range of situations in which emotional responses of the partner can be observed. What is most relevant here is what behavior the subject actually shows, the most likely behavioral pattem to be displayed according to situational characteristics.

In summary, in spite of some methodological weaknesses such as sample size and the fact that the study was restricted to a university environment, this investigation shows a certain influence of situational characteristics on expression of Type A behavior and anger/hostility, and on perception of social support, and demonstrates the possible effects of agreement-disagreement in different environmental settings on cardiovascular health indicators. Social support emerged as a variable closely associated with elevated blood pressure, suggesting that future research should also encompass protective factors rather than focusing only on negative risk factors for cardiovascular health. 


\section{CHAPTER 4}

\section{CONSTRUCT VALIDITY OF TYPE A BEHAVIOUR PATTERN IN VENEZUELA ${ }^{3}$}

\subsection{SUMMARY}

The main objective of the study was to evaluate the prevalence of Type A Behaviour Pattern (TABP) in Venezuela in a sample of employed men $(\mathrm{N}=100)$ and women $(\mathrm{N}=131)$ and to examine whether the concept applies in a Latin American cultural context. To test the validity of the construct the associations of TABP with anger-expression indicators are explored. Participants completed the Structured Interview (SI) for Type A and completed a questionnaire on Type A behaviours based on the Structured Interview items (Bages, Falger \& Warwick-Evans, 1997), and the Anger-expression Scale, from the State-Trait Anger Scale (STAS) to assess anger-expression styles (anger-in, anger-out, and anger-control). A prevalence of $57.4 \% \%$ Type As $(A 1+A 2)$ and $42.6 \%$ Type Bs was found in this group. Females were more often assessed as Type As than males according to the SI. When evaluating Type A components by means of the questionnaire, females scored higher on the time pressure/impatience factor. Type A, according to the SI, was associated with Anger-out, non-verbal hostile gestures, and time-pressure. However, the relationship with Anger-out was significant in males only and the association with time pressure was significant only in females. In summary, Type A behaviour pattern can be reliably assessed in Venezuela. The origins of the qualitative gender difference are discussed.

${ }^{3}$ Bagés, N. . Feldman, L., Chacón, G., Guarino, L. \& Pérez, M.G. (1999), Construct validity of. Type 


\subsection{INTRODUCTION}

Interest in the possible role of psychosocial factors on health and illness has evidently increased in the last decades. Nevertheless, the part that cultural and geographical determinants may play in disease prevention is a highly neglected issue in the literature. The contribution of these factors is of critical importance where psychosocial risk is concerned. In the particular case of coronary artery diseases (CHD), research has shown that there is an important number of personal characteristics that are related to the onset and recurrence of myocardial infarction (MI) either directly or in association with biological risk factors. Those characteristics range from health habits (e.g., smoking, diet, exercise) to behavioural and emotional styles (e.g. Type A behaviour, anger/hostility). None of these are free from cultural and regional influences

Understanding the role of personality, behaviour, and environment in the development of CHD has greatly increased since the emergence of the Type A Behaviour Pattern (TABP) in the United States. TABP was shown to predict MI and other cardiac events (Cooper, Detre \& Weiss, 1981; Matthews \& Haynes, 1986) and more recently the roles of anger and hostility have been widely acknowledged as the most likely toxic elements of TABP (Smith \& Pope, 1990; Smith, 1992; Allan \& Scheidt, 1996). Although some negative findings (e.g. Ragland and Brand, 1988a, 1988b, Shekelle, Hulley, Neaton, Billings, Borhani, Gerace. Jacobs, Lasser, Millermark, \& Stamler, 1985) have diminished the interest in TABP as a risk factor for CHD, these have been attributed to inadequate methods of diagnosis based mainly on self-reports. (Friedman, Fleishnnann \& Price, 1996).

From a transactional point of view (Smith \& Anderson, 1986; Smith, 1989), behavioural and emotional responses are linked to environmental demands, which may include cultural characteristics, child-rearing practices, and specific modes of social interactions. For instance, a strong argument concerning the importance of social and environmental influences on the development of the Type A behaviour Pattern (TAEP) comes from Van Egeren (1991), who discussed the fundamentally contradictory nature of Type A behaviour as being at the same time rewarding and punishing. Van Egeren states that throughout North American history, starting in the $19^{\text {th }}$ century and up to the present day, social forces have acted to mould behaviour patterns in relation to value systems and personal virtues, thereby constantly changing the rewarding and punishing consequences associated with behaviours such as punctuality perseverance, and hard working. Likewise, 
it may be argued that TABP, and related behaviours such as anger-expression, are differentially influenced by their cultures, and that their prevalence, interrelationships with, and influences on, cardiovascular health may vary in different regions of the world, depending upon prevailing social, economic, and political determinants (Eyer, 1977; Eyer, 1980; Bracke \& Thoresen, 1996).

The study of psychosocial factors in the aetiology of CHD occupies many pages of the scientific literature. However, these investigations have been restricted to the United States and Europe. There are few studies on psychosocial risk factors for CHD in Latin America. In a study about Type A hehaviours in high-ranking managers in Caracas; (Venezuela), $82 \%$ were classified as Type A according to the original Structured Interview (Bagés, Feldman \& Chacón, 1995). Moreover, TABP was associated with hypertension, which is not commonly found in other studies (e.g., Mathews and Haynes, 1986).

Renault De Moraes, Swan, and Cooper (1993) conducted a study comparing social factors and health status in Brazil and Great Britain and found that Brazilian white-collar workers scored higher on Type A behaviours, experienced more stress, and reported less use of adequate coping strategies than was reported in normative data from British workers. Also, Brazilians had higher levels of physical and mental illness. Although some methodological drawbacks were acknowledged, these comparative data support the view that the Latin American samples were at disadvantage both in terms of risk factors and selfreported end-points such as hypertension and coronary heart disease.

Similar to the industrialized world, CHD is the leading cause of morbidity and mortality in most of Latin America. Hence, it seems most relevant to evaluate psychosocial factors that have been found to be related to CHD in other cultural backgrounds. Specific lifestyles (such as health habits) and behaviour patterns (such as Type A or anger expression styles) that have been associated with CHD may vary from culture to culture thus having a different impact on emerging diseases. The first step is to test the validity of the TABP construct and to see whether the Type A concept applies to a Latin American culture, Venezuela specifically, where, although a "western" like lifestyle seems to predominate, there are still local social norms, (female and male roles, work values), cultural (social networks), and economic and political patterns which may be strong enough to influence individual behavioural styles. It is not possible at this stage to assess the extent of that influence upon risk behaviours or cognitive styles.

Thus, the objectives of the present study are 1) to explore the prevalence of Type A behaviour in a group of employed men and women in Venezuela, 2) to examine the construct validity of TABP by looking at its associations with anger-expression styles and 
Type A components, 3) to examine the association of Type A and self-reported health indicators, and 4) as culture may be an important determinant of gender differences, to examine data by gender.

\subsection{METHOD}

\section{Participants and procedure}

Data was collected at a University in Caracas, Venezuela, where all administrative staff were notified about the "Health. A Project about the risk of CHD". The study was offered as a free, preventive service to university employees. From the approximately 610 employees, $231(38.5 \%)$ responded to the invitation [100 males, $43 \%$, mean age of 40.5 $(\mathrm{SD}=9.4$ ) years, and 131 females, $56 \%$, mean age of $38.9(\mathrm{SD}=8.4)$ years]. An information campaign promoting the evaluation of health was developed to attract employees. As part of this study participants attended to the SI interview for Type A (described below) and completed the questionnaires on Type $A$, anger expression styles and socio-demographic and health indicators.

\section{Instruments}

To evaluate the various behavioural manifestations of Type A behaviours and anger-expression styles in a comprehensive fashion, the following instruments were used:

Struciured Interview (SI): A Spanish version of The Structured Interview Protocol (Chesney, Eagleston \& Rosenman, 1980), which takes about 20 minutes to conduct, was used. The interview is a diagnostic procedure that allows listening of verbal responses and observation of psychomotor signs, that are typical of the Type A behavior Pattern. There were five interviewers. Dr Ray Roserıman trained three of them, while the remaining two received their training from the other interviewers. Participants were classified as either Type A.1, Type A2, or Type B. Classification was, based on the opinions of the trained interviewers and on the specific instructions 
provided on the original protocol. All interviews in this study were independently corated by another trained interviewer; inter-rater reliability was 94.44

Personal Relations Questionnaire (to assess TABP and Anger expression):

This 34 item questionnaire consists of two sub-scales measuring Type A behaviours and anger-expression modes (Bages, Warwick-Evans, and Falger, 1996). Items (9) in the Type A scale were based on the questions which are part of the SI. It has three sub-scales described as, "Time Pressure/Impatience" (e.g. such as doing two things at the same time or becoming irritated when behind a slow car), "Competitiveness" (e.g. such as enjoying competence at work or being ambitious) and "Non-Verbal Expressions" (e.g. having fast speech or emphatic gestures). Reliability of the Type A sub-scale was .62. To assess anger expression the Anger-Expression Scale of the State-Trait Anger Scale (STAS) (Spielberger, Jacobs, Russell \& Crane, 1983) was used which measures three styles of anger expression, "anger-out", "anger-in" and "anger-control". A Spanish version was used. Reliability coefficients for each anger sub-scale were $.75, .78$, and .69 , respectively (Feldman, 1995)

3. Sociodemographic and Health Indicators Questionnaire. This questionnaire included the following sociodemographic items: age, marital status, number of children, and educational level. The health indicators assessed here were: smoking, physical exercise, hours of sleep, times in hospital in the last 5 years and past 6 months, work absenteeism for health reasons, medical and psychological consultations in the last 6 months, and self-perception of health (scale from 1=very poor health to $5=$ excellent health).

\subsection{RESULTS}

\section{Statistical analyses}

The participants are first described in terms of sociodemographic and health characteristics. Prevalence of Type A according to the Structured Interview and of its components according to the scale is presented separately for males and females. Comparisons between men and women, and also between Type As and Type Bs, about the components of Type A behavior and styles of anger expression will be made using by $\mathrm{t}$ - 
tests. For the analysis of the relationship between Type A and anger expression styles, chisquare tests will be used.

\section{Sociodemographic and Health Characteristics}

These can be seen in Table 4.1. Most participants had an intermediate level of education (secondary school) although the proportion of low educated participants (having only primary or part of secondary school), was relatively high $(23 \%)$. Examination of health habits shows that the percentage of smokers was similar to that of the epidemiological figures for Venezuela as a whole (34\%, Ministry of Health report, 1998). Few people exercised regularly (30\%). In addition, a high proportion of participants (49\%) reported what could be considered an appropriate number of hours of sleep (7-9 per night). Nevertheless, there were an elevated percentage (39\%) of those sleeping fewer hours (4-6 per night). Other health indicators in Table 4.1. suggest, in general, that the participants were healthy.

\section{Type A Behaviours and Anger-Expression}

Table 4.2. shows the distribution of Type A (i.e. A1+A2) and Type B behaviour, as assessed by means of the SI. The prevalence of Type A was $57.4 \%$. Females exhibiting Type A behaviour according to the Interview significantly outnumbered males $(65.5 \%$ versus $44.6 \%$; chi-sq. $=6.06 \mathrm{p}=.01$ ). In Table 4.3. questionnaire scores for Type $\mathrm{A}$ behaviour and anger-expression measures according to gender are depicted. Females reported being more "time pressured/impatient" than males $(t=2.48 ; p=.01)$ but did not differ from males on any of the other measures. 
Table 4.1. Sociodemographic and Health characteristics of the sample (*)

\begin{tabular}{|c|c|c|c|}
\hline & & $\mathbf{n}$ & $\%$ \\
\hline \multirow[t]{3}{*}{ Education } & Low & 68 & 30 \\
\hline & Intermediate & 108 & 46 \\
\hline & High & 54 & 23 \\
\hline \multirow[t]{2}{*}{ Marital Status } & w/partner & 153 & 34 \\
\hline & w/out partner & 78 & 76 \\
\hline \multirow[t]{2}{*}{ Gender } & Males & 99 & 43 \\
\hline & Females & 132 & 57 \\
\hline \multirow[t]{3}{*}{ Age } & Young(-36) & 74 & 32 \\
\hline & Interm. $(37-42)$ & 74 & 32 \\
\hline & Older(43-83) & 83 & 36 \\
\hline \multirow[t]{2}{*}{ Smoking } & Yes & 161 & 70 \\
\hline & No & 70 & 30 \\
\hline Hours of & 4-6 hrs & 90 & 39 \\
\hline \multirow{2}{*}{ Sleep } & $7-9 \mathrm{hrs}$ & 112 & 49 \\
\hline & 10 or more & 4 & 2 \\
\hline \multirow[t]{2}{*}{ Exercise reg. (1/week or more) } & Yes & 67 & 30 \\
\hline & No & 141 & 61 \\
\hline In bed & Zero days & 126 & 55 \\
\hline \multirow[t]{2}{*}{ Last 6 months } & 1 to 9 & 60 & 26 \\
\hline & 10 to 45 & 8 & 8 \\
\hline \multirow[t]{3}{*}{ Self-reported health scale } & Deteriorated & 21 & 9 \\
\hline & Good & 93 & 40 \\
\hline & Very Good & 93 & 40 \\
\hline \multirow{4}{*}{$\begin{array}{l}\text { Work Absenteeism last } 6 \\
\text { months }\end{array}$} & Zero days & 110 & 47 \\
\hline & 1 to 3 & 52 & 22 \\
\hline & 4 to 9 & 23 & 10 \\
\hline & 10 to 45 & 12 & 5 \\
\hline
\end{tabular}

( ${ }^{*}$ In some cases percentages do not add to 100 due to missing values)

There were no associations of Type A behaviour, "anger-out", "anger-in", or "anger-control" with marital status, education or number of children for either males or females. No significant differences were found between Type As living with a partner or not. 
Table 4.2. Percentages of Type $A$ and Type Behaviour for all participants, males and females.

\begin{tabular}{cccc}
\hline SI & $\begin{array}{c}\text { All } \\
\%\end{array}$ & $\begin{array}{c}\text { Males } \\
\%\end{array}$ & $\begin{array}{c}\text { Females* } \\
\%\end{array}$ \\
\hline TypeA1 & 11.2 & 7.0 & 13.8 \\
Type A2 & 46.2 & 36.8 & 52.9 \\
Type B & 42.7 & 56.1 & 33.3 \\
\hline "p $>$.05 & & &
\end{tabular}

Table 4.3. Descriptives of Type A questionnaire global scores, Type $A$ components scores, and Anger expression styles for all participants and separated by gender.

\begin{tabular}{lccccc}
\hline & $\begin{array}{c}\text { All } \\
\text { Mean/SD }\end{array}$ & $\begin{array}{c}\text { Males } \\
\text { Mean/SD }\end{array}$ & $\begin{array}{c}\text { Females } \\
\text { Mean/SD }\end{array}$ & $\mathrm{t}$ & $\mathrm{p}$ \\
\hline Type A - Total score & $13.91 / 4.32$ & $13.71 / 4.30$ & $14.06 / 4.33$ & -.60 & 54 \\
Impatience/time pressure & $4.13 / 1.43$ & $\mathbf{3 . 8 6 / 1 . 4 6}$ & $\mathbf{4 . 3 3 / 1 . 3 7}$ & -2.48 & .01 \\
Competitiveness & $2.68 / 1.79$ & $2.82 / 1.77$ & $2.56 / 1.79$ & 1.10 & .27 \\
Non-verbal & $5.22 / 2.87$ & $5.12 / 2.93$ & $5.28 / 2.82$ & -.43 & .67 \\
Anger - out & $6.29 / 3.38$ & $5.97 / 3.38$ & $6.54 / 3.61$ & -1.24 & .21 \\
Anger - in & $6.29 / 3.35$ & $6.13 / 3.51$ & $6.41 / 4.10$ & -.54 & .59 \\
Anger - control & $16.96 / 4.66$ & $17.32 / 4.93$ & $16.66 / 4.42$ & 1.05 & .29 \\
\hline
\end{tabular}

With respect to Type A measures from the SI (Table 4.4.), "anger-out", "time pressure/impatience" and "non-verbal Type A behaviors" differentiated Type As form Type Bs. Assessing gender differences (Table 4.5.) it was found that Type A males scored higher on "anger-out" than Type Bs. In females, however, there were no significant differences between Type As and Type Bs with respect to any anger expression measure. When looking at the Type A sub-scales, it was found that Type As according to the SI, had higher scores than Type Bs. Moreover, Type A women had significantly more "time pressure/impatience" than Type B women and Type A men had higher "anger-out" scores than Type B men. 
In general, these results show that Type A behavior pattern seems to be a valid concept in Venezuela but that there are specific gender differences with respect to Type A characteristics.

Table 4.4. Differences in anger indicators between Type As and Type Bs (Structured Interview) and Type A questionnaire components

\begin{tabular}{lcccc} 
& Type A & Type B & p & t \\
\hline Anger-in & 6.79 & 5.75 & 1.61 & .11 \\
Anger-out & 7.24 & 5.37 & 3.35 & .01 \\
Anger-control & 16.38 & 17.01 & -.78 & .43 \\
$\begin{array}{l}\text { Type A } \\
\text { tTime-pressure }\end{array}$ & 4.41 & 3.57 & 3.48 & .01 \\
$\begin{array}{l}\text { Type A } \\
\text { competitiveness. }\end{array}$ & 2.87 & 2.39 & 1.65 & .10 \\
$\begin{array}{l}\text { Type A } \\
\text { non-verbal }\end{array}$ & 5.97 & 4.39 & 3.43 & .01 \\
\hline
\end{tabular}

Table 4.5 Differences in anger expression and Type A components according to Type A (SI) for men and women

\begin{tabular}{|c|c|c|c|c|c|c|c|c|}
\hline & \multicolumn{3}{|c|}{ Men } & \multirow[b]{2}{*}{$p$} & \multicolumn{3}{|c|}{ Women } & \multirow[b]{2}{*}{ p } \\
\hline & Type A & Type B & $t$ & & Type A & Type B & $t$ & \\
\hline Anger-in & 6.69 & 5.86 & .87 & .38 & 5.84 & 5.64 & 1.31 & .19 \\
\hline Anger-out & 7.72 & 4.83 & 3.17 & .00 & 7.01 & 5.96 & 1.28 & .20 \\
\hline Anger-control & 16.87 & 17.56 & .53 & .60 & 16.15 & 16.42 & .26 & .78 \\
\hline \multicolumn{9}{|l|}{ Type A } \\
\hline time-pressure & 4.04 & 3.38 & 1.63 & .11 & 4.58 & 3.76 & 2.63 & .01 \\
\hline \multicolumn{9}{|l|}{ Type A } \\
\hline competitiveness & 2.84 & 2.41 & .96 & .34 & 2.89 & 2.36 & 1.29 & .20 \\
\hline \multicolumn{9}{|l|}{ Type A } \\
\hline non-verbal & 6.32 & 4.29 & 2.98 & .01 & 5.81 & 4.50 & 2.17 & .03 \\
\hline
\end{tabular}




\section{Psychosocial and health variables}

Self-reported health indicators were explored in relation to Type A behavior, and the two styles of anger expression that have been found in the past to be more strongly associated with cardiac health, "anger-in" and "anger-out" (Siegman, Dembroski \& Crump, 1992; Mendes de Leon \& Meesters, 1991; Julkunen, Salonen, Kaplan, Chesney \& Salonen, 1994). Due to the gender differences previously found in Type A, these were analyzed separately for men and women. Results are depicted in Table 4.6. In males Type A behavior was related to times admitted to hospital in the past 5 years whereas in females Type A was negatively related to the number of times absent from work due to health reasons.

With respect to styles of anger expression, "anger-in" was related to negative selfconcept of present health in both, males and females. In women, moreover, the association between "anger-in" and work absenteeism approached significance very closely. "Anger" out" was associated to more health indicators but only in men. Males in this case showed associations of "anger-out" with number of days in bed in the past 6 months, and with work absenteeism for health reasons.

Table 4.6. Associations between psicosocial and health variables by gender.

\begin{tabular}{|c|c|c|c|c|c|c|}
\hline & & Smoking & Exercise & $\begin{array}{l}\text { Bed last } 6 \\
\text { mths. }\end{array}$ & $\begin{array}{l}\text { Self- } \\
\text { health }\end{array}$ & $\begin{array}{l}\text { Work } \\
\text { absent. }\end{array}$ \\
\hline $\begin{array}{l}\text { Type } \\
\text { A(SI) }\end{array}$ & $\begin{array}{l}\text { Men } \\
\text { Women }\end{array}$ & $\begin{array}{l}\chi^{2}=.29 \\
\mathrm{p}=.084 \\
\chi^{2}=2.60 \\
p=.10\end{array}$ & $\begin{array}{l}\chi^{2}=1.30 \\
\mathrm{p}=25 \\
\chi 2=0.92 \\
p=.76\end{array}$ & $\begin{array}{l}\chi^{2}=.308 \\
\mathrm{p}=.57 \\
\chi^{2}=2.56 \\
p=.27\end{array}$ & $\begin{array}{l}\chi^{2}=.97 \\
p=.61 \\
\chi^{2}=15 \\
p^{2}=.92\end{array}$ & $\begin{array}{l}\chi^{2}=4.43 \\
\mathrm{p}=.21 \\
\chi^{2}=11.47 \\
p=.00\end{array}$ \\
\hline Anger-in & $\begin{array}{l}\text { Men } \\
\text { Women }\end{array}$ & $\begin{array}{l}\chi 2=.083 \\
\mathrm{p}=.77 \\
\chi^{2}=.3 .63 \\
p=.54\end{array}$ & $\begin{array}{l}\chi^{2}=.78 \\
\mathrm{p}=.37 \\
\chi^{2}=.509 \\
p=.47\end{array}$ & $\begin{array}{l}\chi^{2}=14 \\
\mathrm{p}=.92 \\
\chi^{2}=2.26 \\
p^{2}=32\end{array}$ & $\begin{array}{l}\chi^{2}=9.02 \\
\mathrm{p}=.010 \\
\chi^{2}=6.26 \\
p=.043\end{array}$ & $\begin{array}{l}\chi^{2}=3.34 \\
\mathrm{p}=.34 \\
\chi^{2}=7.65 \\
p=.053\end{array}$ \\
\hline Anger-out & $\begin{array}{l}\text { Men } \\
\text { Women }\end{array}$ & $\begin{array}{l}\chi 2=.140 \\
\mathrm{p}=.707 \\
\chi 2=.07 \\
p=.78\end{array}$ & $\begin{array}{l}\chi 2=2.99 \\
p==.083 \\
\chi 2=.09 \\
p=.75\end{array}$ & $\begin{array}{l}\chi=21.32 \\
\mathrm{p}=.000 \\
\chi 2=2.83 \\
p=.24\end{array}$ & $\begin{array}{l}\chi^{2=3.58} \\
p=.160 \\
\chi 2=4.24 \\
p=.11\end{array}$ & $\begin{array}{l}\chi^{2=20.71} \\
\mathbf{p}=.000 \\
\chi^{2}=2.84 \\
p=4 I\end{array}$ \\
\hline
\end{tabular}

$\chi, \mathrm{p}=$ men

$\chi_{p}=$ women 


\subsection{DISCUSSION}

Findings in this study show that the prevalence of global Type A behaviour pattem, according to the SI, in a sample of employees in a Latin American culture, seems to be comparable to that found in other cultures $(57.4 \%)$. Self-reports of time pressure and nonverbal Type A/hostile behaviours are consistent with findings of the SI. Moreover, the measure of "anger-out" from the scale was significantly higher in Type As further indicating the validity of the Type A construct in this sample of employed males. "Competitiveness" did not seem to be a relevant element distinguishing Type A from Type B individuals.

A second major finding in this study is that the prevalence of Type A is higher in females $(66.7 \%)$ than in males $(43.8 \%)$. This result does not agree with previous data in the United States (Haynes, Feinleb \& Kannel, 1980; Sprafka, Folsom, Burke, Hahn \& Pirie, 1990) where a gender difference was reported of men more frequently having Type A behavior than women. In contrast, Corzine, Butzmen \& Bush (1992) found no gender differences in the TABP. Bages et al (1997), in a study of university employees in the United Kingdom, found that $48.0 \%$ female Type As vs. $76.5 \%$ male Type As. However, the present findings are consistent with data from Croatia (Catipovic-Veselica, Buric, Ilakovac, Amidzic, Kozmar, Durjancek, Skrinjaric \& Catipovic, 1995), and Spain (Valdes and Flores, 1987) where for each of the 3 age groups studied there were also more Type A females than males. Catipovic-Veselica et al., (1995) argue that the rapid changes in social and cultural roles in a developing country such as Croatia, including social pressure to increase familial income, were leading women to work outside the home, and due to multiple roles (mothers, spouses and workers) probably had to assume more Type A related behaviors such as time pressure and impatience.

Moreover, it could be argued that whereas the USA and the UK are highly industrial societies, Venezuela and Croatia may be considered as developing societies, while Spain has recently undergone major economic and social changes. Thus, women may play a different role in societies where social and economic transformations are underway, resulting in a higher prevalence of TABP, as opposed to societies where there has been political, social, and economical stability for a longer period of time.

The economic and political situation at the time of data collection may also be relevant. In the last ten years Venezuela has gone through one of its worst economical and social crises affecting mainly the middle class (Alzate, Llanos, Guerrero, \& Neutra, 1990). 
Perhaps a more active role of women in society is expected and needed during such times leading to more responsibilities and pressures in the daily routine.

Concerning the components of Type A behavior, impatience or time pressure were higher in women, as measured by the Type A questionnaire, and also Type A women had more time pressure indicators than Type Bs, supporting the validity of the SI assessment in women. In previous studies, results regarding gender differences on TABP have been controversial. Wrzesniewski, Forgays \& Bonaiuto (1990) results partially agree with our findings. In testing the generalizability of a new scale about Type A behaviour, they conducted a cross-cultural study in young adults from Poland, Italy, and the United States. Their results showed no statistical differences between sexes in any of the three cultures. All of the Type A factors were present in samples from these countries but the order of the factors varied by sex and culture. In each culture, "Hurying" was a primary factor for females and "Competitiveness" for males; "Hostility" was salient both in Poland and the US, but "Control" was important only to American males.

Type A males have been found to score higher than Type A females in "anger-in" and "anger-control" but no differences were found in "anger-out" using the same instrument as in the present study (Wright, Abbanato, Lancaster, Bourke and Nielsen, 1994). In the Wright et al., study no gender differences were found for "time urgency". In a study conducted by Sprafka et al., (1990) in the USA, women showed lower scores on Type A JAS components, "competitiveness", "time urgency" and "dedication to work". These results are inconsistent with our findings, in that women were more impatient (had more time-urgency indicators) than men and did not differ in competitiveness and non-verbal hostile behaviours.

More time urgency in women than in men in this group may indicate that in females Type $A$ is more related to multiple roles in the daily routine (e.g. "doing two things at the same time", "finishing things as quickly as pessible", "irritated when going behind a slow car"). Women showing such behaviours more often than males may have difficulty in coping with multiple roles, at the work place and at home. In an ongoing study in female Venezuelan professionals, clerical, and blue-collar workers (Feldman, 1995), it was found that interference between work and family life was significantly associated with reports of more physical symptoms, anxiety, depressed affect, and with lower ratings of well-being. Moreover, worrying about interference while at work was associated with reports of less social support and a disturbed relationship with work supervisors. Similar findings have been reported in North American studies (e.g., ONeill \& Zeichner, 1985; Bameth, Davidson \& Marshall, 1991). Also, the fact that Type A was negatively related to work 
absenteeism only for women supports the notion that Type $\mathbf{A}$ behavior in females reflects their involvement with daily obligations rather than their characteristics of anger/hostility.

Type $A$ according to the SI in males, however, seems to be clearly associated with "anger-out" whereas in females this association appears not to be present. This result disagrees with previous studies where no gender differences in anger/ hostility have been found (Weidner, Boughat, Connor, Pieper \& Mendell, 1997; Thomas and Williams, 1991). It is generally agreed that "anger-expression" is a component of Type A behaviour, but here, the association was found mainly in males. Thus, it can be argued that men and women may use different subcomponent routes to achieve it (Wright et al., 1994). Moreover, "anger-out" in women, not differentiating Type As from Type Bs, may indicate that anger-out is not their "toxic factor" and may account for gender differences in risk for CHD. As Wright et al., (1994) point out, Type A women may be psychologically and physiologically healthier than Type A men. Their behavioural patterns may also be due to different environmental demands.

In fact, the associations found here between "anger-out" and health indicators in men but not in women further support the detrimental effects of this anger expression style in males. The present findings for females suggest, on the contrary, that, the suppression of anger could lead to more health problems. This issue deserves further investigation considering other indicators of health beyond self-report.

Response rate may be considered as a limitation of this study. About $40 \%$ of the university employees volunteered to participate, indicating a possible selection bias. If it is assumed that Type As usually do not feel that they have the time to attend to other activities unrelated to work or fill in questionnaires, then it might well be that the prevalence of Type As in this group would have been higher.

In summary, the general prevalence of Type $\mathrm{A}$ in this Latin American group does not seem to differ from that found in other cultures. The percentage found might even be underestimated by the non-responses. The higher percentage of Type A among women appears to be of great interest. Anger-expression scores did not differ for males and females, indicating that this cultural context does not seem to influence how men or women will express their angry feelings.

What seem of importance here is, first, the association of "anger-out" with Type A in men but not in women, together with the dissimilarities in the "impatience/time pressure" factor. Second, the dissimilar associations of "anger-in" and "anger-out" with health indicators are an important issue deserving further attention in future studies. 
These results suggest gender specific routes to Type A which may probably be the product of contrasting environmental and cultural demands. Conflicting findings in industrialised and developing countries concerning psychosocial cardiovascular risk factors should be elucidated in future collaborative research projects. 


\section{CHAPTER 5}

\section{MEASURES OF VITAL EXHAUSTION AND THEIR ASSOCIATIONS WITH CORONARY HEART DISEASE RISK FACTORS IN A SAMPLE OF SPANISH- SPEAKERS}

\subsection{SUMMARY}

The main purpose of this research was to study the reliability and conceptual validity of two Spanish language measures of Vital Exhaustion (VE), a short-term risk factor for coronary artery disease (CAD). The English version of the Maastricht Questionnaire for Vital Exhaustion was used to make a Spanish language questionnaire (VEQ) and a Spanish language interview (VEI) whose scoring systems were equal to the original version. These instruments were administered to a sample of healthy working men $(n=100)$ and women $(\mathrm{n}=130)$ in Caracas, Venezuela. Participants also completed questionnaires designed to measure anger-expression and social support and were assessed by means of the Structured Interview to measure Type A Behaviour Pattern (TABP). Information about other CAD-risk factors, such as age, smoking behavior, and health habits, were collected.

High reliability coefficients, .85 and .91 , were found for the VEI and VEQ, respectively. Principal component analysis provided one-factor solutions for each instrument. The pattern of correlations between VE, assessed by the VEI, and other personality characteristics was rather similar to the pattern observed in other cultures. In general significant, but modest, correlations were observed between VE and TABP, anger expression, a negative self-concept, and low social support. We conclude that VE appears to be a meaningful construct in this particular cultural context.

\footnotetext{
4 Bagés, N., Falger, P., Pérez, M.G. \& Appels, A. (1999) Measures of Vital Exhaustion and their associations with coronary heart disease risk factors in a sample of Spanishspeakers Accepted: Psychology and Health.
} 


\subsection{INTRODUCTION}

Among psychological risk factors for coronary artery disease (CAD), vital exhaustion' (VE) appears to be a strong predictor of acute, non-fatal myocardial infarction (AMI) (Appels \& Mulder, 1988; Appels, Falger, \& Schouten, 1993; Falger \& Schouten,1992) and of new cardiac events after successfull coronary angioplasty (Kop, Appels, Mendes de Leon, de Swart \& Baer, 1994). VE has been defined as a complex of unusual feelings of fatigue and loss of energy, increased irritability, and demoralisation (Appels, 1990).

The term "Vital Exhaustion" first emerged after an extensive series of interviews with AMI-patients who consistently reported a variety of common symptoms and feelings of exhaustion and loss of energy prior to AMI. These prevalent symptoms were pooled to form the construct of "Vital Exhaustion" and to formulate the first version of the Maastricht Questionnaire (MQ) to assess VE (37 items) (Appels, 1980). To the 37 original items, 21 new ones were added on the basis of further interviews. This. Form A was employed in a fouryear prospective study, namely, the Rotterdam Civil Servants Study in 3,877 city employees in Rotterdam, the Netherlands. In this study. VE was an independent risk factor for acute, non-fatal AMI with an age-adjusted relative risk of 2.3 over 4.2 years of follow-up (Appels \& Mulder, 1988). This association between VE and first AMI was also demonstrated in a number of case-control studies in men (Falger \& Schouten, 1992; Meesters \& Appels, 1996a; Meesters \& Appels, 1996b; Nelissen-de Vos, 1994) and in women (Appels, Falger \& Schouten, 1993). Based on the discriminatory power with respect to predicting AMI, Form B was defined, which consists of 21 items (Meesters \& Appels, 1996a; Meesters \& Appels, 1996b).

Several methodological shortcomings linked to the questionnaire format, (such as loss of valuable qualitative information, absence of a well-defined time scale that may discriminate long-standing exhaustion as a result of prolonged ailments from fatigue of recent onset, or patients' apparent misinterpretations of items) were found. This resulted in a significant number of "false positives" in some of these studies, for which reason an interview schedule was arranged. This schedule was named the "Maastricht Interview for Vital Exhaustion" (MTVE), and it features 23 questions (Meester \& Appels, 1996a; Meesters \& Appels, 1996b). The MQ identifies more subjects as "vitally exhausted", that is, more "false positives", than the MTVE according to Meesters \& Appels, (1996a). Moreover, the latter discriminates better between MI-cases and controls, (Meesters \& Appels, 1996a). 
The issue of generalizability of cardiac risk factors across cultures is a most important one and deserves special attention. "Vital Exhaustion" has been studied in The Netherlands and in some other European countries (e.g. in Finland, Keltikangas-Järvinen, Raîkkônen \& Hautanen, 1996; in Sweden, Kristensen, Kucinskiene, Bergdahkd, Calkauskas, Urmonas \& Orth-Gomér, 1998; in England, Bennett, Smith, \& Gallacher, 1996). It seems important to examine the role of VE in the pathogenesis of CAD in other cultural and geographical areas such as Venezuela, where, as in most industrialised countries in the world, CAD is the primary cause of death in both men and women, accounting for some $42 \%$ of allcause mortality. (Venezuelan Ministry of Health. Report, 1994)

Vital Exhaustion is a concept developed to describe the mental precursors of myocardial infarction. As Meesters \& Appels (1996b) have pointed out, there is no gold standard to which the questionnaire method and the interview method to assess VE can be compared. Therefore, the cross-cultural validation of an instrument has to rely upon similarities in the nomological network of a concept. This means that similar correlations with other personality characteristics have to be observed as they are observed in other countries. For example, Falger (1989) found a significant correlation between VE and the Type A Behavior Pattern (TABP). Meesters and Appels (1996a,b), on the other hand, found that the interview method of assessment of VE (MIVE) shows substantial correlations with "hostility". One should expect similar correlations in Venezuela if the instrument assesses Vital Exhaustion. A similar pattern of correlations is considered to support the cross-cultural validity of the VE concept.

Vital Exhaustion shares a number of symptoms with depression. Therefore, one might expect a positive correlation between an instrument to assess Vital Exhaustion and "negative self-concept", as indicator of depression. However, the correlation between VE and "negative self-concept" is expected to be of low magnitude because VE is not identical to depression. Van Diest \& Appels (1991) have shown that exhausted but otherwise healthy subjects complained about tiredness and loss of energy but did not report a "depressed mood", which is a key component of depression.

Up to now no studies have been published investigating the association between VE and social support. As social support is considered to buffer the effects of stress one may expect that those who do not feel supported by their environment are at an increased risk of becoming exhausted.

Thus, the research questions addressed in this study are: 1 . What is the reliability of two methods used to assess VE in Venezuela?, and 2. Do these instruments show similar 
correlations with some relevant personality characteristics (TABP, "anger-expression", "negative self-concept", and lack of social support) as observed in other countries?

Spouses of the participants were asked to respond to the TABP, 'anger-expression', and social support questionnaires with respect to the study participants. They had had the opportunity to observe the behaviour of their partners for a long time. In previous studies, reports from significant others have been found to be good predictors of Type A behaviours, blood pressure variability, and CHD in study participants (Atchinson \& Condon 1994; Bagés, Warwick-Evans \& Falger, 1997). Thus, in the present study, it was considered relevant to include reports from spouses on Type A and the anger expression styles of their partners.

\subsection{METHOD}

\section{Participants and Procedure}

In a joint project between the Netherlands, and Venezuela, the revised version (21 items plus 2 on irritability) of the MQ (Meester \& Appels, 1996a) was adninistered to a sample of personnel from the administrative staff of the Simon Bolivar University (Caracas, Venezuela). All (610) administrative staff was notified of the details of "Proyecto Salud: Empleados USB“" (Health Program: USB Staff) a project collecting, psychosocial, biochemical, nutritional, and anthropometric data about CAD-risk. In order to recruit employees for the project an information campaign promoting the evaluation of health was developed. Of the total number of employees, 230 (38.5\%) responded to the invitation [100 men, $43 \%$, mean age $=40.5(\mathrm{SD}=9.4)$ years, and 130 women, $56 \%$, mean age $=38.9(\mathrm{SD}=8.4)$ years]. Data on VE was collected employing the Spanish version of the Maastricht Questionnaire, named the Vital Exhaustion Questionnaire (VEQ) and the same questionnaire, administered as an interview schedule, named the Vital Exhaustion Interview (VEI) (see below for description of the instruments). Cardiac health indicators and health habits, and TABP, 'angerexpression', and social support were also assessed by means of questionnaires as described below.

All 230 participants. completed the questionnaires and $142(62 \%)$ attended the interviews. In the majority of cases (approximately $80 \%$ ) non-attending the interview was due. to not being able to agree on a time to attend given the limited number of hours available for interviews. In the minority of cases (approximately $20 \%$ ) participants refused to attend. There 
were no significant differences in age or gender between those who attended the interviews and those who did not. There was a one-week time gap between the two evaluations. Approximately $65 \%$ of the participants responded first to the questionnaire before attending the interviews and the remaining $35 \%$ attended the interviews first and then answered the questionnaire. Practical reasons did not allow splitting the group randomly and using two different sequences to control for order effects. Nevertheless, the order of presentation was different for a relatively high proportion of the participants.

The VE interview and the Structured Interview Protocol (SI) (Chesney, Eagleston \& Rosenman, 1980) were carried out by the first and third authors and by two research assistants. In addition, 67 spouses ( $35 \%$ of total living with a partner) returned questionnaires about the characteristics under study of the participants.

There were no significant differences in any of the sociodemograhic variables or health indicators between those employees who participated in the study and those who did not participate.

\section{Instruments}

1.Vital Exhaustion: Questionnaire (VEQ) and Interview (VEI). The English version of Form B, which contains 23 items, formed the basis of the Spanish translation, carried out by a Spanish native speaker. The Form B version was the first to be reported with complete psychometric data (Meesters \& Appels, 1996a; Meesters \& Appels, 1996b). Local idiomatic expressions were used to convey the same meaning of English questions did (such as, "Do you believe that you have come to a "dead end"?; "Siente que he llegado a un callejon sin salida"?). Both instruments (VEQ and VED) were back translated into English by a second translator (English native speaker) to ensure that item contents were as similar as possible (Appendix 1). Both our instruments were identical in contents but differed in method of administration and scoring. The questionnaire featured the same scoring system of the original MQ [i.e., three categories: "No, absence of feeling" (0); ? = "Uncertain about its presence over the last six months" (1); and "Yes, presence of feeling" (2)]. Participants received this questionnaire along with all other study questionnaires. The interview items were identical to the ones in the questionnaire but featured a different scoring method (as in the original interview) with only two categories: "Absence of feeling" (0) and "Presence of feeling" (1). Doubtful answers about the presence or absence of specific feelings over the last six months were removed 
by discussing each response with the corresponding participant, thereby avoiding any misinterpretations; of the questions. Thus, possible response range was $0-46$ for the questionnaire and 0-23 for the interview.

\section{Personal Relations Questionnaire (Social Support/Anger) and Type A Structured} Interview. This composite questionnaire consists of two sub-scales, one on social support which had been employed in a prior British study (Bagés, et al., 1997) which included 18 items from the Social Support Questionnaire (Flemming, Baum, Gisriel \& Gatchel, 1982), and the Anger-expression Scale, from the State-Trait Anger Scale (STAS) (Spielberger, et al.,1985), which included three factors, anger-out, anger-in, and anger-control. In the case of the sub-sample that attended the VE Interview $(\mathrm{N}=142)$, Type A Behaviour was assessed by means of the Structured Interview Protocol (Chesney, Eagleston \& Rosenman, 1980). According to the original protocol, participants were classified as either Type A1 or A2 (Coronary Behaviour), or Type B (Non-Coronary Behaviour).

3.Personal Relations Questionnaire for Spouse/Partner. This questionnaire is identical to the previous one, except for the pronouns which refer to the participants. In this way, the spouse/partner would provide information about overt behaviours typical of their partners with respect to anger-expression and social support.

4. Positive and Negative Self-concept. This is a combined, shortened version of the Automatic Thoughts Questionnaire (Ingram \& Wisnicki, 1980), which originally contained 30 items on negative thoughts and 30 items on positive thoughts. Our shortened version contains 5 positive and 5 negative items with respect to Self-concept. These were the highest loading items after factor analysis with loadings ranging from .67 to .78. (Appendix 2).

5. Sociodemographic Data, Health Indicators, and Health Habits Questionnaire. This questionnaire includes self-reported data on marital status (living or not with a partner), formal education (1=primary and some secondary school, $2=$ secondary completed, and $3=$ higher education), work absenteeism for health reasons, self-concept of health (from $1=$ very poor to $5=$ excellent), previous illnesses, visits to physicians and psychologists, hospitalisations, and days in bed due to illness over the past 6 months, smoking history, physical exercise, and hours of sleep. 


\section{Statistical Methods}

The psychometric properties, of the VEQ and VEI were assessed by means of Cronbach's Alpha reliability coefficients to test internal consistency of both instruments. The possible number of dimensions thereof was explored by performing a Principal Components Factor Analysis. The cut-off point for item removal was .30. The correspondence between the VEQ and the VEI was tested by using Pearson product-moment correlation coefficient ( $r$ ) and also by using chi-square tests when scores were divided in tertiles to explore extreme values. The associations of the VEQ and VEI scores with the psychosocial variables, TABP, angerexpression and sociall support, was explored using Spearman Correlation Coefficients (due to the skweness of the VEQ and VEI distributions). One-way ANOVAS were used to test the associations of VE with sociodemographic variables and health indicators. Different numbers of participants in some tables are due to missing values in view of the different number of respondents to the VEQ and the VEI and to not fully completed questionnaires.

\subsection{RESULTS}

\section{Reliability of VEQ and VEI}

Reliability analysis of the Spanish Vital Exhaustion Questionnaire revealed that all items from the original scale, except for item 9, ('disrupted sleep) showed an item-total correlation $>30$. Thus, item 9 was eliminated from further analysis. The new 22 item scale showed good reliability $(\alpha=91)$. Results from a principal component factor analysis showed a clear one factor structure, with item loadings $>.40$ (Table 4. 1, left column), which accounts for $36.6 \%$ of total variance. Eigen values showed a sharp decrease between the first and the second factor ( 8.20 to 1.79 ) indicating that there is one factor only. These statistics indicate that the Spanish questionnaire measures an internally consistent construct, which is similar to the original Dutch version (Meesters \& Appels, 1996a,b). 
Table 4.1. Items and Factor Loadings of the Vital Exhaustion Questionnaire (VEQ) and Vital Exhaustion Interview (VEI) (LISTWISE, 1 Factor Solution)

\begin{tabular}{lcc}
\multicolumn{1}{c}{ ITEMS } & VEQ $^{*}$ & VEI** \\
\hline 01. Tired & .546 & .340 \\
02. Listless & .646 & .555 \\
03. Lack of energy & .650 & .365 \\
04. Increased irritability & .587 & .437 \\
05. Feeling dejected & .707 & .549 \\
06. Empty battery & .553 & .339 \\
07. Inability to accomplish & .565 & .434 \\
08. Waking up exhausted & .616 & .357 \\
09. Disrupted sleep & .292 & .280 \\
10. Inability to cope & .574 & .326 \\
11. Give up trying & .555 & .608 \\
12. Being annoyed & .611 & .535 \\
13. Blow up easily & .537 & .632 \\
14. Decrease in energy & .712 & .552 \\
15. Loss of libido & .405 & .490 \\
16. Difficulty solving mental task & .615 & .343 \\
17. Difficulty concentrating & .582 & .448 \\
18. Shrink from work & .701 & .259 \\
19. Reaching dead end' & .643 & .724 \\
20. Feel like crying & .484 & .600 \\
21. Feeling defeated & .665 & .692 \\
22. Hopelessness & .615 & .722 \\
23. Death wish' & .641 & .673 \\
\hline
\end{tabular}

*: Eigen Value: $8.05 \overline{7}$; Explained Variance: $36.6 \%$

**: Eigen Value: 5.850; Explained variance: $27.9 \%$

Similarly, reliability analysis of the Spanish Vital Exhaustion Interview revealed that of the 23 original items, all but items 9 ('disrupted sleep') and 18 ('shrink from work'), showed an item-total correlation $>30$. Thus, items 9 and 18 were removed from further analysis. The new 21 item schedule showed good reliability $(\alpha=85)$. Principal Component Factor Analysis also showed a one factor structure, with item loadings $>.32$ (Table 4.1., right column), explaining $27.9 \%$ of total variance. Eigen values also showed a sharp drop between the first and the second factor ( 6.57 to 2.00 ) indicating only one factor. Thus, the present Spanish versions of the questionnaire and interview to measure vital exhaustion both measure an internally consistent construct. 


\section{Correspondence between Questionnaire and Interview}

The product-moment correlation between these instruments was highly significant $(r=.32 \mathrm{p}<.0001)$; however, the magnitude of this correlation was lower than expected for two similar instruments differing mainly in response mode. A scatter-plot was made to explore the relationship between the scores of the VEQ and the VEI. Inspection showed that, taking the mean and $1 / 2$ SD for the MQ (8) and the MIVE (14) as cut-off points (Meesters \& Appels, 1996a), there were considerably more false positives (26\%) (fourth quadrant) than false negatives (11\%) (first quadrant). This was confirmed when the scores of both instruments were divided in tertiles (Table 4.2.). Twenty-nine percent of those rated as highly exhausted (third tertile) according to the questionnaire were classified as nonexhausted (first tertile) according to the interview. By contrast, only $8 \%$ of those rated as exhausted with the interview were classified as non-exhausted when using the questionnaire. These results indicate that the questionnaire classified more participants as exhausted than did the interview, so that in the case of people with high scores on the questionnaire, further assessment with the interview may be necessary.

Table 4.2. Correspondence of scorings with the VEQ and VEI. Scores divided in tertiles $(n=231)$.

\begin{tabular}{cccc}
\hline $\begin{array}{c}\text { Vita| Exhaustion } \\
\text { Interview }\end{array}$ & \multicolumn{1}{c}{$\begin{array}{c}\text { Vital } \\
1\end{array}$} & $\begin{array}{c}\text { Exhaustion } \\
2\end{array}$ & $\begin{array}{c}\text { Questionnaire } \\
3\end{array}$ \\
\hline 1 & $22(46.8 \%)$ & $11(23.4 \%)$ & $14(29.8 \%)$ \\
2 & $20(35.5 \%)$ & $23(39.7 \%)$ & $15(25.9 \%)$ \\
3 & $3(8.1 \%)$ & $14(37.8 \%)$ & $20(54.1 \%)$ \\
\hline
\end{tabular}

Vital Exhaustion and other Psychosocial Risk Factors

According to the SI, 82 (57\%) of the participants were Type A and $60(43 \%)$ were Type B. Diagnosis was based on the SI protocol instructions (Chesney et al.,1980). When Type As and Type Bs were compared by means of a two-tailed t-test, 
Type As, showed higher scores, on exhaustion according to the VEI $(t=2.17, \mathrm{p}=, 033)$ but not when assessed by means of the VEQ $(t=.63, p=.532)$.

Table 4.3. shows the associations of VEQ and VEI with positive and negative self-concept, anger-expression styles, and social support. In general, the interview (VED) showed more, and stronger, associations with these psychosocial variables than did the questionnaire (VEQ). In particular, negative self-concept was correlated with both instruments. All anger measures, with the exception of anger-out by the spouse, showed associations with VEI-scores. With the VEQ, this was the case only for spouse-reported anger-out: Social Support was negatively associated with the VEI but not with the VEQ.

Vital Exhaustion, socio-demographic variables and self-reported health indicators

Age groups differed in exhaustion scores according to the VEI indicating that exhaustion tended to be higher in younger individuals. This was not true for the VEQ (Table 4.4.)

On comparing men and women on VE it was found that women were more exhausted than men when assessed by the VEI.

Exhaustion scores, on either the VEI or VEQ, were not significantly different in terms of educational level but there was a tendency in that direction when assessed by means of the VEI.

With regard to health indicators, VEI scores were related to times in hospital, days in bed for health reasons, and work absenteeism for health reasons during the past six months, whereas no associations were found with the VEQ.

\section{Gender differences}

Inspection of the reliability coefficients and factor analyses showed that the factor structure and the reliability were almost similar in both genders.

Finally, it is interesting to note that in female participants, there were no associations of VE with health indicators. In men, however, VEI scores were related to both work absenteeism and days in bed over the last six months (F. $=4.75$, $p=.005$; $\mathrm{F}=.9 .28, \mathrm{p}=.003$, respectively). 
Table 4.3. Spearman Correlation Coefricients of the Vital Exhaustion Questionnaire (VEQ) and Vital Exhaustion Interview (VEI) with Self-concept, Anger-Expression, and Social Support.

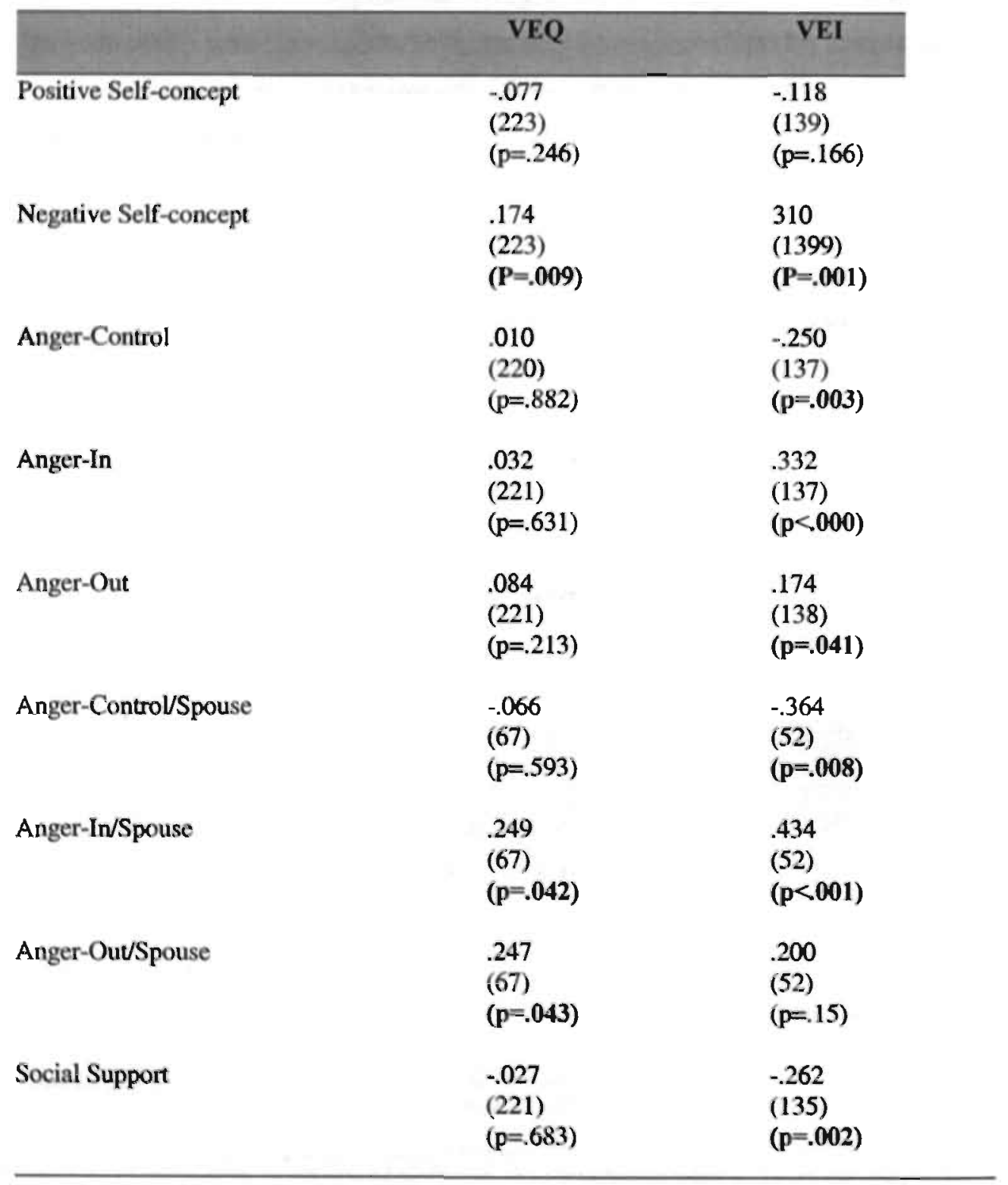


Table 4.4. Two-tailed t-tests or one-way ANOVAS to assess differences in VEQ and VEI scores according to demographic and health indicators.

\begin{tabular}{|c|c|c|c|c|c|c|c|c|c|}
\hline & & & VEQ & & & & VI & EI & \\
\hline & & $\mathbf{n}$ & Mean/SD & t/F & p & $\mathbf{n}$ & Mean/SD & $t / F$ & p \\
\hline Age & $\begin{array}{l}\text { Young(-36) } \\
\text { Interm.(37- } \\
42) \\
\text { Older(43-83) }\end{array}$ & $\begin{array}{l}74 \\
74 \\
83\end{array}$ & $\begin{array}{l}11.4 / 9.4 \\
10.0 / 9.4 \\
11.6 / 9.8\end{array}$ & .62 & .53 & $\begin{array}{l}46 \\
45 \\
51\end{array}$ & $\begin{array}{l}4.36 / 4.6 \\
2.86 / 3.2 \\
2.19 / 2.6\end{array}$ & 4.63 & .01 \\
\hline Gender & $\begin{array}{l}\text { Males } \\
\text { Females }\end{array}$ & $\begin{array}{l}99 \\
132\end{array}$ & $\begin{array}{l}10.8 / 9.8 \\
11.2 / 9.4\end{array}$ & .34 & .73 & $\begin{array}{l}65 \\
77\end{array}$ & $\begin{array}{l}2.1 / 2.6 \\
3.9 / 4.1\end{array}$ & 2.99 & .00 \\
\hline Education & $\begin{array}{l}\text { Low } \\
\text { Intermediate } \\
\text { High }\end{array}$ & $\begin{array}{c}68 \\
108 \\
54\end{array}$ & $\begin{array}{l}11.5 / 9.5 \\
10.8 / 9.9 \\
11.2 / 9.1\end{array}$ & .110 & .89 & $\begin{array}{l}39 \\
72 \\
30\end{array}$ & $\begin{array}{l}4.1 / 4.1 \\
2.9 / 3.6 \\
2.2 / 2.7\end{array}$ & 2.59 & .07 \\
\hline Marital Status & $\begin{array}{l}\text { w/partner } \\
\text { w/out partner }\end{array}$ & $\begin{array}{c}153 \\
78\end{array}$ & $\begin{array}{c}11.6 / 10 . \\
0 \\
9.9 / 8.4\end{array}$ & 1.30 & .18 & $\begin{array}{l}92 \\
50\end{array}$ & $\begin{array}{c}3.1 / 3 \\
2.9 / 3.3\end{array}$ & .32 & .75 \\
\hline Smoking & $\begin{array}{l}\text { No } \\
\text { Yes }\end{array}$ & $\begin{array}{c}160 \\
70\end{array}$ & $\begin{array}{c}11.3 / 10 . \\
2 \\
10.7 / 8.8\end{array}$ & -.52 & .60 & $\begin{array}{l}78 \\
64\end{array}$ & $\begin{array}{l}2.8 / 3.8 \\
3.3 / 3.3\end{array}$ & .83 & .40 \\
\hline $\begin{array}{l}\text { Hours of } \\
\text { Sleep }\end{array}$ & $\begin{array}{l}4-6 \text { hrs } \\
7-9 \text { hrs } \\
10 \text { or more }\end{array}$ & $\begin{array}{c}90 \\
112 \\
4\end{array}$ & $\begin{array}{c}11.0 / 8.9 \\
11.0 / 10 . \\
1 \\
5.5 / 7.0\end{array}$ & .65 & .52 & $\begin{array}{c}55 \\
73 \\
2\end{array}$ & $\begin{array}{l}3.073 .2 \\
2.9 / 3.6 \\
4.0 / 2.8\end{array}$ & .11 & .88 \\
\hline $\begin{array}{l}\text { Medical } \\
\text { consultations } \\
\text { last } 6 \text { mths }\end{array}$ & $\begin{array}{l}\text { Zero } \\
1-3 \\
3 \& \text { more }\end{array}$ & $\begin{array}{l}89 \\
86 \\
17\end{array}$ & $\begin{array}{c}10.9 / 8.8 \\
10.3 / 10 \\
1 \\
14.4 / 10 . \\
5\end{array}$ & 1.20 & .29 & $\begin{array}{c}57 \\
53 \\
8\end{array}$ & $\begin{array}{l}2.4 / 2.9 \\
3.5 / 4.1 \\
4.2 / 2.9\end{array}$ & 1.90 & .14 \\
\hline $\begin{array}{l}\text { Hospital } \\
\text { last } 6 \text { mths }\end{array}$ & $\begin{array}{l}\text { Zero times } \\
1 \\
2\end{array}$ & $\begin{array}{c}199 \\
5 \\
3\end{array}$ & $\begin{array}{c}10.6 / 9.5 \\
8.7 / 3.9 \\
10.1 / 5.8\end{array}$ & 2.20 & .11 & $\begin{array}{c}127 \\
4 \\
-\cdots\end{array}$ & $\begin{array}{l}2.85 / 3.1 \\
6.7 / 8.5\end{array}$ & 5.03 & .02 \\
\hline $\begin{array}{l}\text { In bed } \\
\text { Last } 6 \text { mths }\end{array}$ & $\begin{array}{l}\text { Zero days } \\
1 \text { to } 9 \\
10 \text { to } 45\end{array}$ & $\begin{array}{c}126 \\
60 \\
8\end{array}$ & $\begin{array}{c}10.4 / 9.5 \\
11.1 / 9.4 \\
16.6 / 12 . \\
2\end{array}$ & 1.56 & .21 & $\begin{array}{c}83 \\
35 \\
5\end{array}$ & $\begin{array}{c}2.32 / 2.9 \\
3 \\
4.0 / 3.5 \\
5.6 / 7.8\end{array}$ & 4.80 & .00 \\
\hline $\begin{array}{l}\text { Work } \\
\text { Absenteesm } \\
\text { last } 6 \text { mths }\end{array}$ & $\begin{array}{l}\text { Zero days } \\
1 \text { to } 3 \\
4 \text { to } 9 \\
10 \text { to } 45\end{array}$ & $\begin{array}{l}110 \\
52 \\
23 \\
12\end{array}$ & $\begin{array}{l}10.1 / 9.5 \\
11.6 / 8.8 \\
9.7 / 10.5 \\
17.0 / 9.8\end{array}$ & 2.13 & .09 & $\begin{array}{c}73 \\
33 \\
12 \\
7\end{array}$ & $\begin{array}{l}2.2 / 3.0 \\
3.5 / 3.3 \\
4.6 / 3.6 \\
2.8 / 2.0\end{array}$ & 2.68 & .04 \\
\hline
\end{tabular}




\subsection{DISCUSSION}

Two central issues were addressed in this investigation. The first one dealt with the psychometric characteristics of Spanish versions of two Vital Exhaustion instruments: the questionnaire and the interview. It was found that the Cronbach's coefficients of both are good. Factor analysis revealed a robust one-factor solution for the final versions of both forms of evaluating VE, similar to the original Dutch versions (MQ and MIVE). The similar results found for men and women strengthen the reliability of both instruments. VE also showed to have good construct validity as assessed through its associations with other psychosocial factors such as anger-expression, negative self-concept, and secial support. Thus, the pattern of correlations of VE and other psychosocial factors in Venezuela is similar to the pattern of associations found in Holland. These results add to the generalizability of the VE construct across cultures

An important aspect to take into account is that the final versions of the questionnaire and interview herein presented, are slightly different form the original ones due to the omission of item 9 in the questionnaire and items 9 and 18 in the interview. Nevertheless, this does not seem to affect the internal consistency of the instruments. But what about the Vital Exhaustion construct? The decision to eliminate those items, and specially item 9, was strictly meant to follow statistical criteria due to their low item-total correlation. It could be argued that, on theoretical grounds, being important elements of the original construct, such items might have been preserved as part of the VE instruments, in special item 9. Nevertheless, a more conservative position was adopted here.

The question remains as to whether sleep disturbances are representative of VE in this sample, as it has been reported in previous studies (Falger, Schouten, Appels and Nelissen-de Vos, 1988; Falger \& Nelissen-de Vos, 1997). More recently, in a review study, Falger \& Nelissen-de Vos (1997) reported that sleep problems are related to exhaustion and seem to be present prior to MI. It is interesting to note that item 9 was one of the three most frequently reported in the VEI $(26.8 \%)$. Yet, it had a very low factor loading both in the VEQ and the VEI. It should also be mentioned that there was no association either between exhaustion scores and number of hours of sleep. Cultural differences in sleeping pattems may account for the main difference observed with respect to the original studies. In fact, hours of waking up and going to bed in Venezuela seem to differ from those in European countries. In addition, geographical differences in terms of latitude (e.g. hours of daylight, temperature) may possibly explain the discrepancy. Further attention in future studies should be given to this question. 
The low magnitude of the association between the VEQ and the VEI was surprising.

This may reflect the substantial difference often observed between questionnaire and interview measures used in cardiovascular research (Thoresen and Powell, 1992). It is obvious that questionnaires and standardized interviews have their advantages and limitations. Interviews are vulnerable to subjective elements in their administration and in interpretation of the response. On the other hand, the interview gives the interviewer the opportunity to check the presence or absence of each symptom by asking if the answer is unclear. In case the respondent does not know he/she might endorse the question mark on the questionnaire, which elevates the sum score. In case the respondent does not know or cannot decide between "yes" or "no", the interviewer may ask additional questions.

It is of special importance to note that the original form of the MQ did not clearly specify how long a complaint should be present to score as an indication of the presence of a state of exhaustion. VE is thought to reflect a breakdown in adaptation to stress. Therefore, subjects suffering from a chronic or long-lasting fatigue should not be considered to be exhausted. The advantages of the interview over the questionnaire and the need to specify the duration of the complaints led to the construction of the MIVE. In contrast with the MQ complaints with a duration of more than 18 months are scored as "complaint is absent". It was observed that the MIVE discriminated much better between MI cases and coronary controls that the MQ did (Meesters \& Appels, 1996b).

In the present study both the VEQ and the VEI correlated significantly with negative self-concept and anger-in and with anger-out as assessed by the spouse. Except for the correlation of VEQ and anger-out as assessed by the spouse all correlations were higher when Vital Exhaustion was assessed by the interview. As expected, VE, correlated with the TABP, anger-in, anger-out and low social support, but only when Vital Exhaustion was assessed by the interview. So, the conclusion with regard to the second research question is: Vital Exhaustion shows the same pattern of correlations with other personality characteristics as observed in The Netherlands but only when the interview method is used to assess VE.

Regarding the associations of VE with other psychosocial variables that have previously been related to CHD it was found that, In agreement with previous findings, Type As in this group yielded higher scores of exhaustion in the VEI than Type Bs. When assessed by the VEQ this association was not found. In Meesters and Appels (1996b) study Type As had higher scores on exhaustion than Type Bs in both healthy controls and MI patients. Falger (1989) also reported this association in males, both healthy and MI patients. He proposed that Type As become "vitally exhausted" as a result of their overloading and over-commitment to work. 
Anger expression was also related to VEI scores and to a much lesser extent to the VEQ. In fact, only spouse reports showed correlations with the VEQ. Although not of a large magnitude, the consistent association of VE with anger-in, anger-out, and, negatively, with anger-control, suggests that inappropriate anger modes of expression are important concomitants of the process of exhaustion and validate the irritability sub-component of the vital exhaustion construct.

As said in the introduction, Vital exhaustion shares a number of symptoms with depression but is believed to be distinct from depression because the key component of a depressed mood is usually not observed in exhausted individuals. The results of the present study give some support to this belief. A positive self-concept was not associated with VE and the correlation of VE with negative self-concept was modest. In previous studies exhaustion and depression were found to share from $25 \%$ to $50 \%$ common variance depending on the method of assessment (Klein, Stokhof \& Cohen, 1994). In this study negative self-concept was assessed by rather strong items (among them: "all my efforts are worthless", "I am good for nothing", "nobody understands me"). The modest correlation coefficient of .31 between VE and negative self-concept observed in this study indicates that the common variance between VE and a strong negative self-appreciation does not extend $10 \%$.

Younger participants reported higher VE. This finding is in accordance with previous results found in groups of MI patients and controls but stronger in the first (Meesters \& Appels, 1996b) where age was negatively associated with VE. Regarding cardiac patients Meesters and Appels (1996b), point out that the common attribution of their fatigue and tiredness to age is usually incorrect.

Unexpectedly, smoking was not related to exhaustion and this is opposite to previous findings (Meesters and Appels, 1996b). At this stage, we cannot account for this as yet.

In agreement with previous studies (Appels, et al., 1993), women had higher scores on exhaustion than men when assessed by the VEI. This result may be related to previous findings in this same cultural context where a higher proportion of Type A women than men was found (Bagés, Falger, Feldman, Chacón, Pérez \& Guarino, submitted). Moreover, exhaustion in women could be related to multiple roles in the daily routine. However, exhaustion was not related to any of the self-reported health indicators in women as it was in men which might indicate gender differences in the repercussion of exhaustion upon health.

In sum, the present study has shown that both the questionnaire and the interview forms to assess VE have a good reliability. It also shows that a similar pattem of correlations between VE and other personality characteristics was observed in Venezuela as in The Netherlands, in case VE was assessed by an interview. Therefore, we have decided that it 
would be worthwhile to test the validity of Vital Exhaustion as precursor of myocardial infarction in a case-control study and to use the interview method for the assessment of VE. This study is underway. 


\section{CHAPTER 6}

\section{VITAL EXHAUSTION AS A RISK FACTOR OF MYOCARDIAL INFARCTION: A CASE-CONTROL STUDY IN VENEZUELA ${ }^{3}$}

\subsection{SUMMARY}

In this study, 32 first MI cases, and 42 healthy controls were compared with respect to Vital Exhaustion, a state characterised by loss of energy, increased irritability and feelings of demoralisation. This state has been found to precede the onset of cardiac events. Participants also responded to questionnaires on Type $\mathrm{A}$ behaviour, anger expression (angerin, anger-out, and anger-control) and positive and negative self-concept. Results showed that VE discriminated well between MI patients and controls $(\mathrm{OR}=15.42(95 \% \mathrm{C} . \mathbf{I}=3.92$ 60.67)) even when controlling for age, smoking, and exercise. The Odd ratio decreased to 12.34 when controlling for socio-economic status. Groups also differed in anger-in but not in anger-control, anger-out, negative or positive self-concept. Anger-in was correlated to VE in all participants pointing to the relevance of withholding emotions in relation to exhaustion. Exhaustion was strongly associated with negative self-concept in the $\mathrm{MI}$ cases group but significantly discriminated between cases and controls when adjusted for negative selfconcept. Summarising, the results show that, as has previously been found in other countries, in Venezuela VE is a precursor of MI.

\footnotetext{
${ }^{5}$ Bages, N, Appels, A. \& Falger, P. Vital Exhaustion as a risk factor for coronary heart disease: A case-control study in Venezuela. In press. International Journal of Behavioral Medicine.
} 


\subsection{INTRODUCTION}

One of the most challenging issues in preventive cardiology is the recognition of precursors of cardiac problems such as Myocardial infarction (MI). The possibility of identifying short-term indicators of the possible occurrence of a major heart problem would enhance the chances of life in many individuals.

In contrast to the well known biological (hypertension, faulty lipid metabolism and obesity) and behavioural (smoking and lack of exercise) risk factors which do not give any indication about the imminence of the attack, pointing only to the increased possibility of its occurrence in the future, Vital Exhaustion, a state characterised by feelings of fatigue and loss of energy together with irritability and demoralisation (Appels, Falger and Shouten, 1993) may indicate that an MI could occur within a relatively short period of time. Among the well known psychosocial risk factors for Coronary Heart Disease (CHD), (TABP, hostility and lack of social support,) it is the only one that has shown to be a short-term risk indicator of MI in several studies (e.g. Rotterdam Civil Servants Study, Appels \& Mulder, 1988).

It has been estimated that between 50 to $60 \% \%$ of MI patients report excess fatigue and depression-like symptoms in the months previous to the cardiac event (Kop, et al., 1994). Vital exhaustion is the syndrome that tries to describe those feelings preceding a heart attack. Several case-control (Meesters \& Appels, 1996; Appels; Falger \& Schouten, 1992; Kop, et al., 1994) and prospective studies (Rotterdam Civil-Servants Study, Appels \& Mulder, 1988) carried out in Europe have shown those symptoms to be present during a short period, approximately between 1 and 6 months in half of the cases, and between 1 and 10 months in $80 \%$ of the cases (Meesters \& Appels, 1996b) previous to the MI. Moreover, vital exhaustion symptoms were found to be independent of the severity of the underlying coronary artery disease and to predict new cardiac events after successful PTCA (Kop, et al., 1994).

The hypothesis that MI is the possible consequence of an "emotional drainage" has been proposed, emphasising that it may be the result of life conflicts that are beyond personal control such as unemployment or death of loved ones (Falger \& Schouten, 1992). In addition, maintained job stress, poor personal relations at work, and especially overtime, have also been proposed as possible causes of the fatigue and sadness (Falger \& Schouten, 1992). Individuals under those circumstances remain in a state of alert in order to be able to deal with difficult external or internal demands leading to overexertion of the cardiovascular system.

VE has also been linked to other psychosocial risk factors such as Type A behaviour pattern (TABP), (Falger, 1989; Meesters \& Appels, 1996b) and inadequate styles of angerexpression and hostility (Meesters \& Appels, 1996b). These findings probably indicate that 
Type As become exhausted as a result of over-commitment to work and a frantic lifestyle. In the Meesters \& Appels (1996) studies it was found that hostility was associated with the Buss-Durkee hostility Inventory and several dimensions of the Cook-Medley Hostility Scale.

Due to the influence of culture on cognitive, affective, and behavioural patterns, the universality of effects of psychosocial factors, and the background where they exert their influence on health, need to be evaluated. In a previous study concerning the adaptation of Vital exhaustion instruments into the Spanish language and the construct validity of the construct in a Latin American culture, it was found that VE was strongly related to angerexpression indicators and to a measure of depressive thinking (negative self-concept) in a working population. Contrary to previous findings, no associations with TABP global measures were found. On the other hand, sleep disturbances, a complaint commonly found among the vitally exhausted, and differentiating MI cases and controls, was not associated with VE (Falger et al., 1988)

The objectives of the present study were to compare MI patients and controls in terms of exhaustion symptoms in a Latin American context. Anger expression, negative and positive self-concept and social support were also evaluated. Research questions were focused on the role of VE as a risk indicator of MI and to the psychological correlates of VE. Of major importance in this study is the within and between groups exploration of the relationship of VE with anger-expression (in, out and control) and especially with negative self-concept as a measure of low self-esteem and guilt.

\subsection{METHOD}

\section{Participants and Procedure}

Seventy-four men participated in the study, 32 of them, the case group, were either hospitalised or recently discharged with a diagnosed first MI in a major University Hospital in Caracas, Venezuela. Selection was based on diagnosis of the MI and patients were interviewed sequentially after reading the daily hospital records for admissions or discharges. Discharged patients were interviewed during their first or second control visit to the hospital. None of the patients approached refused to participate in the study (100\% response rate). Diagnosis was based on clinical criteria decided by the cardiologist, ECG readings, and cardiac enzyme levels (SGOT, CPKmb). Forty-two men with no history of CHD formed the control group that was drawn from a larger group working as administrative personnel in a University in the same city. Attempts were made to have comparable percentages of case and 
control participants in each educational level (primary, secondary and higher) by excluding employees in the higher educational levels. Also, groups were made comparable in age by excluding those that were younger than the youngest case and those that were older than the oldest case. The mean age of the control group was 47.7 years and for cases 50.6 years.

Controls were asked how they felt during the last six months and cases how they felt in the six months previous to their heart problem. Most patients were interviewed while in hospital a few days after the MI. A few were interviewed during the second or third week after their MI while attending a cardiac rehabilitation program at the same hospital. Participants in the control group were interviewed about Vital Exhaustion and were given the questionnaires. These instruments were returned a few days later. Some of them failed to respond to all items and for this reason, some of the analyses are based on different numbers of controls. They were all volunteers participating in a larger project on health issues. Interviewers were blind with regard to the purposes and hypothesis of the study. With respect to comparability of the samples, the control group was drawn from university employees who were likely to use the services of the same type of hospital as the one the cases were drawn from.

\section{Psychological measures}

1. Vital exhaustion. In both samples, data on Vital Exhaustion was collected using the Spanish versions of the Vital Exhaustion Interview (VEI) that consisted of 21 items. In fact, the VEI is essentially an oral form of the Vital Exhaustion Questionnaire with dichotomized categories. Characteristics of the instrument and the complete psychometric evaluation have been reported elsewhere (Bagés, Falger, Pérez \& Appels, 1998) The items were translated and back translated from the English version. It has two scoring categories, $0=$ absence and $1=$ presence of the symptom. The psychometric study showed that the instrument had a good reliability coefficient $(\alpha=.912)$ and a single factor structure similar to the original Dutch version.

2. Anger Expression. The expression of anger was assessed using the Anger-Expression Scale (Spielberger et al., 1983). This is a 24 item inventory which evaluates the frequency of anger-related experience and behaviour. It has three sub-scales measuring expression of anger towards others or the environment (Anger-out), suppression of experienced anger (Anger-in) and controll over the experience of anger (Anger-control).

3. Social Support. Eighteen items drawn from the Dunn, Putallaz, Sheppard \& Lindstrom scale (1987), were used. The present version included only the items of observable social 
support behaviours. Items based on behaviours, rather than on cognitions or emotions, related to social surport are considered to be more representative of the use that respondents make of their social network either supporting or seeking for support.

4. Positive and Negative Self-concepi. We used a shortened version of the original questionnaire by Ingram and Wisnicki, (1988) which contains 30 items on negative selfconcept or low self-esteem and 30 items on positive self-concept or optimism. The version used here contained 5 positive (my life will improve, everything will work out, it is fun for others to be with me, I am proud of my achievements, my friends appreciate me) and 5 negative items (my efforts are worthless, I am a failure, no one can understand my probiems, I have disappointed others, my future is uncertain). These ten items had the highest loadings in a factor analysis previously conducted.

5. Sociodemographic data, health indicators, and health habits. Data on marital status (living with a partner or not), education (primary only, secondary and some university, university completed), number of children (none, $1-2 ; 2-3 ; 4$ or more), self-concept of health $(1=$ very deteriorated to $5=$ excellent), visits to doctor and psychologist, and hospitalisations (times during the last 6 months), smoking (presence-absence, passive smoker, past smoker), physical exercise (none - once per week or more) and sleep hours $(0-4 ; 5-7 ; 8$ or more), was collected through a questionnaire specially designed for this study.

\section{Statistical Methods}

Data was first examined at a univariate level by looking at group differences in VE, anger-expression measures and social support, and in health habits and health indicators, with t-tests for independent samples and chi-square tests. These analyses, were followed by multivariate tests for the discriminative power of vital exhaustion using multiple logistic regression analyses where status of the participants as MI cases or controls was the dependent variable. In the first model, Vital exhaustion was introduced as a continuous variable first and then as a categorical one dividing scores by the median of the control group (7) into "exhausted" and "non-exhausted". Next, VE was introduced with each of the possible confounding variables, age, self-reported hypertension, smoking, exercise, and educational level separately. This strategy, instead of a simultaneous inclusion, was adopted because the small number of subjects makes the estimation of the risk associated with each factor rather. imprecise when all factors are simultaneously introduced in the model. Bivariate analyses were used to explore the associations between VE and psychosocial variables within each 
group with Pearson product-moment correlation tests for continuous variables and chi-square tests for the dichotomous ones. The data were analysed using SPSS/PC 5.1 licensed to the Universidad Simón Bolívar (1991).

\subsection{RESULTS}

Socio-demographic variables, Health Habits and health indicators

The MI patients and controls did not differ on any of the socio-demographic variables registered but approached significance in age, (Mean MI patients: 50.69 years, Mean Control group: 47.76 years; $\mathrm{t}=1.82 ; \mathrm{p}=.092)$, and educational level, $($ chi-sq=6.51; $\mathrm{p}=.089$ ), Marital status or number of children were not significantly different either (Table 6. 1).

Group differences for health habits and health indicators are also depicted in Table 6.1. Self-perception of health in the MI patients was significantly lower than that of controls during the past six months before their MIs. The two groups did not differ in any of the other self-reported health indicators or habits, previous illnesses or doing exercise and no differences were found either with respect to hours of sleep (chi-sq. $=4.88 ; \mathrm{p}=.188$ )

\section{Vital Exhaustion}

As predicted, the VEI scores were significantly higher in the patient group than in the control group (Table 6. 2. In fach, patients' scores were more than twice as high as controls ${ }^{\circ}$ scores. When VE was dichotomised the OR and $95 \%$ confidence interval were $15.42(3.92 .-60.67)$

Legistic regression analyses showed that $\mathrm{VE}$ was the only variable to remain in all models, that is, when VE was included alone, and when age, smoking and exercise were added. (ORs of 15.42, 17.15, 15.63 and 17.55, respectively). This was also true for all the procedures explored, direct and forwards and backwards, with maximum likelihood ratios. In the case of education a One-way ANOVA showed that there were differences in exhaustion between the three levels of education being those at the lower level more exhausted $(\mathrm{F}=4.32$; $\mathrm{p}=.01$ ). When controlling for education in the model OR decreased from 15.32 to 12.34 . Therefore, low education partially accounted for the VE difference between cases and controls. More specifically, the reduction was 2.98 of the total OR for VE. The estimates for 
VE can be observed in Table 6. 3. Vital exhaustion discriminates very strongly between MI cases and controls.

Table 6.1. Differences in percentages of positive responses to adverse health Indicators and habits.

\begin{tabular}{|c|c|c|c|c|c|c|}
\hline & \multicolumn{2}{|c|}{$\begin{array}{c}\text { Cases } \\
\text { Yes }\end{array}$} & \multicolumn{2}{|c|}{$\begin{array}{c}\text { Controls } \\
\text { Yes }\end{array}$} & \multirow{2}{*}{$x^{2}$} & \multirow[t]{2}{*}{$\mathbf{P}$} \\
\hline & $\mathrm{N}$ & $\%$ & $\mathrm{~N}$ & $\%$ & & \\
\hline Marital status & & & & & 0.14 & .701 \\
\hline Living with partner & 27 & 84.4 & 34 & 81.0 & & \\
\hline Living without partner & 5 & 15.6 & 8 & 18.9 & & \\
\hline Number of children & & & & & 1.58 & .660 \\
\hline 0 & 2 & 6.3 & 3 & 7.1 & & \\
\hline $1-2$ & 8 & 25.0 & 14 & 43.3 & & \\
\hline $3-4$ & 16 & 50.0 & 21 & 50.0 & & \\
\hline 5 or more & 6 & 18.8 & 4 & 9.6 & & \\
\hline
\end{tabular}

Educational level

$\begin{array}{lcccc}\text { Primary and some secondary } & 17 & 53.1 & 12 & 28.6 \\ \text { Secondary finished } & 9 & 28.1 & 18 & 42.9 \\ \text { Higher finished } & 5 & 15.6 & 12 & 28.6\end{array}$

Medical consultations last 6 months

$\begin{array}{lcccc}\text { None } & 14 & 43.8 & 18 & 42.7 \\ l-3 & 15 & 46.9 & 17 & 40.5 \\ 4 \text { or more } & 3 & 9.4 & 1 & 2.4\end{array}$

Psychological consultations last 6 months

$\begin{array}{lcccc}\text { None } & 31 & 96.9 & 38 & 90.5 \\ l-3 & 1 & 3.1 & 1 & 2.4 \\ 4 \text { or more } & 0 & 0 & 1 & 2.4\end{array}$

Self-concept of health

Very poor or poor

Good

Very good or excellent

$\begin{array}{ll}7 & 21.9\end{array}$

9.37

.009

$\begin{array}{ll}7 & 21.9\end{array}$

12.4

$18 \quad 56.3$

$19 \quad 45.2$

Current smoking

$10 \quad 31.3$

$20 \quad 47.6$

Past smoking

22. 68.8

$12 \quad 28.6$

.062

.802

Passive smoking

$25 \quad 78.1$

$\begin{array}{ll}28 & 66.7\end{array}$

.035

.849

Practising Exercise

$7 \quad 219$

$\begin{array}{ll}28 & 66.7\end{array}$

.604

.436

Hypertension

$14 \quad 43.8$

$16 \quad 38.1$

Diabetes 
Table 6.2. Mean score differences between cases and controls in Vital Exhaustion, anger expiression, negative and positive self-concept, and social support

\begin{tabular}{lcccc}
\hline & Cases & Controls & T & P \\
\hline Vital Exhaustion & $7.9(5.61)$ & $2.2(2.41)$ & 5.68 & .000 \\
Negative self-concept & $3.0(3.55)$ & $3.56(3.18)$ & .59 & .555 \\
Positive self-concept & $12.5(2.95)$ & $11.3(2.93)$ & 1.70 & .093 \\
Anger-out & $7.9(5.66)$ & $5.9((3.14)$ & 1.83 & .075 \\
Anger-in & $9.3(4.50)$ & $6.1(3.97)$ & 3.21 & .002 \\
Anger-control & $16.4(6.47)$ & $18.1(4.56)$ & 1.23 & .225 \\
Social support & $34.00(6.54)$ & $33.88(9.31)$ & .06 & .952 \\
\hline
\end{tabular}

Table 6.3. Maximum likehood estimates of Ingistic parameters and confidence intervals for Vital Exhaustion controlling for age, smoking, exercise., and education

\begin{tabular}{lllll}
\hline Variable & B & Se & OR & P \\
\hline Vital Exhaustion & 2.84 & .72 & 17.15 & .001 \\
Age & .078 & .04 & 1.08 & .114 \\
Vital Exhaustion & 2.74 & .70 & 15.63 & .001 \\
Smoking & -.089 & .64 & .914 & .889 \\
& & & & \\
Vital Exhaustion & 2.86 & .72 & 17.55 & .001 \\
Exercise & -1.00 & .67 & .366 & .134 \\
Vital Exhaustion & 2.51 & .77 & 12.34 & .001 \\
Education & -.432 & .38 & 1.27 & .258 \\
\hline
\end{tabular}


Anger, Negative self-concept, and Social Support

Table 6. 2. also shows group differences in all the other psychosocial variables evaluated. Anger-in was significantly higher in the patients group than in the control group. Anger-out and anger-control did not differ in either group, nor did social support.

Table 6.4. shows Pearson product-moment correlations of VE with the other psychosocial variables within each group separately. VE strongly correlates with negative self-concept in the patients but not in the controls, anger-in is related to VE in both groups but anger-out is not. Interestingly, anger-control is inversely related to VE only in the MI cases group. In brief, the associations between VE and other psychosocial risk factors differ for MI patients and controls, especially in the case of negative self-concept and anger-control. However, adjusting the discriminative power of VE for negative self-concept showed that VE discriminated significantly between both groups $(\beta=2.92 ; \mathrm{SE}=.75 ; \mathrm{OR}=18.63 ; \mathrm{p} .001)$. The $\beta$, $\mathrm{SE}$, and OR of negative self-concept were: $-.12, .09$. and $.88(\mathrm{p}=.18)$ in this equation.

It should de pointed out that the number of controls here varies from 38 to 42 due to the fact that some participants did not fully answer some of the questionnaire items.

TABLE 6.4. Product-moment correlations of VE with anger expression scores, negative and positive self-concept, Type $A$ total score, Type $A$ components, and social support for MI cases and controls.

\begin{tabular}{lcc}
\hline & $\begin{array}{c}\text { MI cases } \\
(\mathrm{n}=32)\end{array}$ & $\begin{array}{c}\text { Controls } \\
(\mathrm{n}=38-42)\end{array}$ \\
\hline Negative self-concept & $.457(\mathrm{p}=.008)$ & $.163(\mathrm{p}=.328)$ \\
Positive self-concept & $-.230(\mathrm{p}=.204)$ & $-.267(\mathrm{p}=.104)$ \\
Anger-out & $.299(\mathrm{p}=.096)$ & $.113(\mathrm{p}=.498)$ \\
Anger-in & $.501(\mathrm{p}=.004)$ & $.388(\mathrm{p}=.016)$ \\
Anger-control & $-.460(\mathrm{p}=.008)$ & $-.251(\mathrm{p}=.128)$ \\
Social support & $-.193(\mathrm{p}=289)$ & $-.278(\mathrm{p}=.086)$ \\
\hline
\end{tabular}




\subsection{DISCUSSION}

The main purpose of this study was to explore the discriminative power of Vital Exhaustion between MI cases and controls. Results showed that in Venezuela too, Vital Exhaustion was a precursor of MI. In accordance with previous results in The Netherlands, MI patients had higher total scores on the VE interview than healthy individuals. In fact, an OR of 15.00 is exceptional and probably overestimates the true risk due to the low scores in the control group. Therefore it is prudent to look at the left side of the confidence interval only. This indicates that the risk of VE is with $95 \%$ confidence, at least 3.92 . This is very similar to the results of previous prospective studies (Rotterdam Civil-Servants Study, Appels \& Mulder, 1988). The role of socio-economic status, expressed here in terms of educational level, should be taken into consideration when interpreting those findings. Although there were no significant differences $(\mathrm{p}=, 08)$ between cases and controls in educational level, a closer inspection of the data shows that more cases were less educated and that more controls had higher education. The reduction in the OR for VE when controlling for educational level indicates that education partially explains the VE differences between both groups.

With respect to health indicators and health habits it was surprising to find that the groups did not differ in present or past smoking, hypertension or diabetes. The small sample size may account for this finding. Self-perception of health did differentiate MI cases and controls very clearly. This may have been related to pre MI symptoms. In fact, those participants who were exhausted had lower self-perception of their health than the nonexhausted ones (exhausted: $6.9 \%$ with deteriorated health: non-exhausted $2.9 \%$ with deteriorated health; chi-sq. $=9.06, p=.010$ )

Hostility has previously been found to be associated with VE (Meesters \& Appels, 1996) and although we measured anger-expression and not hostility, the two constructs share common aspects and anger has also been linked to the risk of CHD (e.g. Siegman, et el., 1991). In the present study it was found that MI patients had significantly higher scores in anger-in but not in anger-out indicating that in this sample withholding negative affect had a detrimental effect in cardiac health whereas expression of angry feelings did not. Moreover, anger-in was strongly associated with VE in both groups possibly indicating that exhaustion is linked to this anger-repression behavioural style although it is not possible at this stage to establish a causal connection.

The finding in this study that VE was associated with negative self-concept only in the MI cases is of utmost importance adding information in relation to the conceptualisation 
of the construct. Depression has been associated with CHD in several studies (Fielding, 1991; Barefoot \& Schroll, 1996 (Booth-Kewley and Friedman, 1987; Shekelle and Ostfeld, 1965, Crisp, Queen and DeSouza, 1984). Fielding (1991), refers to VE as a "depression-like" condition and even equates VE and depression concepts. He admits, though, that the heterogeneity in describing depression in relation to MI makes interpretations of that most probably prodromal condition more problematic. What seems to be generally agreed upon is that depressive symptomatology, as related to $\mathrm{CHD}$, is different from depression as described in the DSM-III, that is, different from a psychiatric disorder and more as a mood and physical energy state (Fielding, 1991; Appels, 1997).

According to Meesters and Appels (1996b), VE and depression share an important number of symptoms but mood disturbances and cognitive components of depression (guilt, loss of self-esteem) are usually absent in exhausted individuals. In our study, thoughts related with low self-esteem, personal worthlessness, and personal failure were highly correlated with VE only in persons who recently had a heart attack. Adjusting VE for negative self-concept did not lower the risk of exhaustion. This indicates that the risk of exhaustion cannot be attributed to lowered self-esteem or feeling of personal failure. Thus, the data of this study suggests that VE does not equate depression.

The high value of the odds ratio raises two main issues about the validity of this study. The first possible source of invalidation may have come from information bias and the second one could have been due to the possibility that VE feelings reflect the disease process itself. In the case of the information bias, there are four possible causes. First, information was collected retrospectively with the well-known possible bias of this data collection method. The interviews were carried out in most cases 72 hours after the MI and that time period can be considered as short for memory retrieval of cognitions, affects, and behavioural styles previous to the heart problem. Although this was considered as adequate it could also be argued that participants might have been suffering from the trauma of the recent cardiac event nesulting in memory distortions. Although the possibility exists that in search for meaning patients may have expressed a gloomy view of their feelings before the MI, it was perhaps too early after the event and not yet at a stage of reconstruction for possible causes. It was most probably the first time that those patients had the chance to discuss or talk about their very recent event. Second, the fact that interviewers were not blind to the condition of the MI patients may have also affected results but this is obviously unavoidable and should be taken into account when interpreting results. Third, the interviewers knowing about the hypothesis of the study may have influenced results. To avoid this source of information bias, most interviews were carried out by a trained assistant who was unaware of the study purposes and 
blind to the hypothesis of the study. Fourth, as most of the patients were hospitalised it is possible that the high exhaustion scores were due to feelings associated with hospitalisation. Nevertheless, in previous studies it was found that VE discriminates well between MI patients and sick controls (Falger, 1989).

Regarding the second possible source of bias, a question arises with regard to the possibility of VE as a marker of CHD. VE was found to be predictive of CHD in a cohort of 3600 males free of angina pectoris and past MI at entrance (Appels and Mulder, 1988), Therefore, it is unlikely that exhaustion is predictive of CHD because it is a marker of manifest heart disease. However, this observation cannot rule out the possibility that VE is a marker of sub-clinical heart disease. This question was addressed in a study of VE as risk indicator for a new cardiac event after successful percutaneous transluminal coronary angioplasty (PTCA) (Kop, Appels, \& Mendes de Leon, 1996) and in a study of VE as risk indicator of Sudden Cardiac Death (SCD) (Golombeck, Appels, van Breukelen, de Vreede, \& Gorgels, 1999). In the PTCA study no association between VE and the ejection fraction of the left ventricle was observed. VE was significantly associated with the number of diseased vessels and PTCA resulted in a significant decrease of VE scores. However, although statistically significant, the absolute values of the association also indicated that only a small proportion of the variance in VE scores could be explained by the number of diseased vessels (Kop, Appels, Mendes de Leon, de Swart \& Bar, 1996). In the SCD study, autopsy data of 37 victims of SCD were available. VE experienced in the year before SCD (assessed by heteroanamnestic interviews) was negatively associated with the number of diseased vessels although this association was not significant $(F=1.61 ; p=.21)$. According to modern pathology CHD is based upon an inflammation of one or more corọnary vessels. Recent epidemiological findings have shown that this inflammation might be caused by viral and/or bacterial infections. Part of the inflammation process is formed by the release of cytokines. It is known that cytokines may reach the brain and evoke feelings of tiredness and malaise as part of the homeostatic reaction to the inflammation (Appels, 1997). This evokes the question whether VE is a marker of functional processes in the vessel wall. The Maastricht group is presently testing this hypothesis in angioplasty patients.

In conclusion, VE seems to be a risk factor for first. MI in a Latin American cultural context which is similar to findings in other countries. It is important to consider the strong relationship found with indicators of inadequate anger-expression, specifically anger-in, and further explore possible causal relationships. The association found between VE and indicators of mood disturbances only in MI patients suggests that exhausted patients are more 
prone to depression like-thoughts. This study, which is the first investigating VE in Latin America, indicates that this concept merits further attention. 


\section{CHAPTER 7}

\section{MAIN RESULTS AND GENERAL DISCUSSION}

The results of five studies examining different aspects of psychosocial risk for myocardial infarction have been presented throughout this dissertation. In all these investigations there was a common interest in studying psychosocial risk factors for MI from different perspectives, bearing always in mind the idea that the aetiology of cardiac illness is multi-factorial and that it is important to examine associations between risk factors rather than isolated causes or determinants.

Four of the five studies here reported were conducted in Venezuela and one in England. The four studies conducted in Venezuela represent the first formal and systematic reports in the country about psychosocial risk factors for cardiovascular problems. In general, in Latin America there has been relatively little interest in this area and very few studies are found in the international (English) literature. The few reports known to the author have appeared in the regional (Spanish) scientific literature (Hernandez-Pôzo, Muñoz, \& Serrano, 1996; Hemandez-Pozo, \& Rodriguez-Ortega, 1999) reaching local researchers but failing to contribute to a widespread, world-wide, knowledge in regard to the reality about psychosocial risk and CHD in this part of the world.

Two well-known risk factors, Type A and Vital Exhaustion, were the core elements under investigation but always in association with other psychosocial variables that may contribute to the risk of CHD and particularly of MI. Table 7.1 presents a summary of the results of the five studies.

The strength of this dissertation lies in the multiple aspects approached. For instance, Type A was evaluated in top managers, in university employees (in two countries), and in cardiac patients. Both, males and females were assessed in three of the studies. Moreover, spouses also informed about Type A characteristics in two of the reports and work supervisors in one. Added to this, the associations of Type A with anger, and social support were explored in two of the studies. Type A and cognitive performance at work was assessed in the first study, and finally, blood pressure was measured to study Type A-Type B differences and to look at the associations with reports from different informants. All these measures gave the opportunity to explore Type A from different perspectives.

Likewise, Vital Exhaustion received attention through the adaptation and validation of its measuring instruments, questionnaire and interview, in Spanish and through examining its discriminative power between MI cases and healthy controls in Venezuela. The 
associations of VE with other psychosocial risk factors such as Type A, anger, negative selfconcept, and lack of social support were also of interests in two of the studies.

In all cases, sociodemographic variables were considered in association with Type A and VE. This is of particular interest when assessing psychosocial risk factors in a different cultural context. Gender differences were of particular interest in three of the studies.

In this chapter a brief discussion of all five studies in a global manner will be attempted, bearing always in mind that most of them are carried out in different groups of individuals and had different focus of attention.

Limitations of the studies will be acknowledged adding some recommendations to overcome them. Finally, some suggestions and implications for future studies will be laid out.

\subsection{TYPE A BEHAVIOR PATTERN}

\subsubsection{Prevalence of Type A, culture and SES}

As shown in Table 7.1, the frequency of Type $\mathrm{A}$ in the managers in Caracas was much higher than in the other two groups of university employees. This is in agreement with previous findings in Australia were those at a higher occupational level showed more Type A behaviors (Byrne \& Reinhart, 1989; Corzine et al, 1992)

Although the oil-company where the managers worked is state-owned, their working style corresponds more to that of a privately owned company. Substantial differences between public and private sector employees with regard to organisation and working conditions have previously been reported in the literature (Chrismas, Fry, Reeves, Lewis, Weinsten, 1989). University employees are civil servants and the University organisation is more similar to that of a state-managed organisation, both in England and in Venezuela. It is probable that private companies reinforce or prefer Type A behaviours among their employees. Moreover, working hours are known to be longer for those at higher positions and Type $\mathrm{A}$ has been previously found to be related to long working hours (Sorenson, Jacobs, Pirie, Folsom, Luepker \& Gilum, 1987). All this may partially account for the differences found in Type A prevalence in the different groups.

Some results from previous studies conducted in Venezuela support this idea. Type A was evaluated, by using the SI, in women in middle managerial positions (Micale, 1992), in women working as university employees (Meneses, 1994), and in policemen 
(Yanes, 1992), The prevalence of Type A in these three groups was $57 \%, 53 \%$ and $35 \%$, respectively, indicating a gradient in Type A prevalence related to occupational position.

Within the university organisation, however, results seem contradictory. In the British study Type A university employees had a higher educational level than Type Bs whereas no differences were found between Type As and Type Bs in the Venezuelan sample of university employees. The results in the Venezuelan group seem to be opposite to the general finding that Type $\mathrm{A}$ is associated with educational level (Baker, Dearborn, Hastings, \& Hamberger (1984); Moss, Dielman, Campanelli, Leech, Harian, Van Harrison \& Horvath, 1986). It seems possible then that in the Venezuelan higher education institutions Type A characteristics may not be the most relevant aspect for promotion. Although we did not assess Type $\mathrm{A}$ according to occupational level within the university it is safe to assume that those with a higher educational level held higher occupational positions. Thus, although in general a gradient in Type A according to occupational level seems to exist in Venezuela, within a particular organisation such as a university, Type A was not related to educational level. These results emphasise the relevance of environmental demands on the display of Type A characteristics and support the notion that behavior patterns should be observed in their psychosocial contexts (Orth-Gomer \& Undén, 1990).

Previous studies comparing Type A in different cultures support some of our findings. Using the Jenkins Activity Survey to evaluate Type A in a sample of 1682 employees in a Japanese company, Hayano. Takeushi, Yoshida, Jozuka, Mishima \& Fujinami (1989) found that their mean scores were similar to those found in the WCGS (USA). Managers had higher scores on all Type A factors than technicians, clerks or skilled labourers. Moreover, those with higher education were also more Type A than those with only high-school education, whereas factor $\mathrm{H}$ (high-driving) was higher in those with the lowest educational level, consistent with North American studies. There were interesting differences in Factor J (job-involvement) of the JAS in that in Japan this factor did not include items conceming improvement or promotion in the past years, indicating that these aspects are not so much related to Type A behaviour in Japan, perhaps partially accounting for their lower incidence of CHD. The prevalence found among the male Japanese employees was $40 \%$, which is much lower than that of Venezuelan managers and also lower than that of the two university employees' samples in England and Venezuela. It even seems to be more comparable to the prevalence of Type A policemen in Caracas (35\%) who have a very low educational level. Cultural differences in the display of Type A behaviours seem evident in these results, nevertheless, the possibility exists that differences 
are partially accounted for by the use of different assessment methods for Type A (Jenkins Activity Survey in Japan and Structured Interview in the other studies).

\subsubsection{Type A, Cognitive performance, and blood pressure}

Blood pressure was evaluated in studies 1 and 2 (Table 7.1). In study 1 it was found that there were no differences in SBP, DBP or heart rate between Type As and Type Bs during two cognitive tasks but only in the last phase of the experimental session just before leaving the room. At that time, Type As showed significantly higher SBP than Type Bs. The first part of these results is in agreement with Hayano et el., (1989) who found no correlations of Type A with SBP, DBP, serum cholesterol, triglycerides, smoking, uric acid, blood glucose in Japanese employees. Moreover, Williams, et al., (1991) found no Type A Type B (SI) differences in BP reactivity in middle-aged men when they were completing a mental arithmetic task and during a sensory intake task.

Taking a final measure just before leaving the experimental room is not a procedure usually reported in the psychophysiology or risk factors literature and experimental sessions generally end after the last reactivity measure. By the time that measure was taken, participants had already been debriefed and were ready to leave and return to their own offices. The significant Type A-Type B difference found in SBP may indicate that for Type As it is difficult to "unwind" after confronting a situation perceived as stressful, in other words they were unable to decrease their cardiovascular response to reach again their baseline level after finishing the tasks in spite of reporting lower levels of subjective tension.

The hyper-reactivity hypothesis so frequently proposed to explain the link between Type A and CHD may be extended to include not only the physiological over-responding to stressful stimuli but also the inability to retum to baseline levels once the stressing stimulus is no longer present (Manuck, 1994).

Although the tasks did produce a heightened subjective response due to their difficulty, it may be necessary to evaluate Type A-Type B differences in the work environment using job related tasks such as decision making or conflict solving in a quasireal manner so that Type As may be more prone to feel the challenge and probably exhibit more pronounced cardiovascular responses.

In the study described in Chapter 3, carried out in university employees in England and hence a very different type of sample, no differences between Type As and Type Bs were found either in blood pressure measures taken before during and after the SI, which 
may be considered as a stressful situation. Unfortunately, no recordings were carried out just before leaving the room and hence, no comparisons can be made with the previous study. However, it is important to note that in both studies, with two types of task and in two different samples, Type As and Type Bs did not differ in their BP reactivity during a stressful task, confirming previous reports.

The potential use of other informants to report about psychosocial risk factors and biological indicators was made evident in another part of this same last study (Chapter 3). It seems of great importance the fact that the reports of the participants themselves, their spouses, or work supervisors on Type A, anger, or social support were not associated with $\mathrm{BP}$, but the level of disagreement between informants was. Results showed that there were significant associations between disagreement about social support between participant and supervisor and indicators of elevated blood pressure (maximum diastolic blood pressure, MAXDBP, minimum systolic blood pressure, MINSBP, and minimum diastolic blood pressure, MINDBP). Moreover, disagreement about perceived level of TABP between participant and spouse was associated with elevated MAXDBP. That is, those with lower selfperception of social support and those with lower self-perception of TABP than their spouse or supervisor, and, thus, negative levels of agreement, had higher BP values. No other reports about disagreement levels and cardiovascular measures or cardiac end-points have been found in the literature, thus it is not possible to compare the present findings with previous results.

\subsubsection{Type A, Anger Expression, and Social Support}

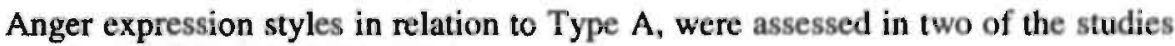
reported here and in relation to social support in one. In Chapter 3 results showed that there were significant positive relationship between TABP and anger according to assessments by the participants, their spouses and their work supervisors indicating a stability of this association among different environments. In the following study (Chapier 4), TABP was again found to be related to anger, and with non-verbal behaviours indicating hostility, but only in males. Several differences between these two reporits limit the possibility of interpreting the results regarding anger jointly. Namely, studies were conducted in two different cultures and different instruments were used to assess anger expression. It is possible to reason, though, that Type $\mathrm{A}$ and anger are closely related but that aspects such as gender and anger-expression style may modify this relationship. 
Findings in previous studies have shown that research on anger is controversial although with few exceptions (Ranchor, Sanderman, Bouma, Buunk \& van der Heuvel, 1997) the most common general finding is that inadequate forms of anger expression may be damaging for cardiac health, and that perception of social support is a protective factor for cardiovascular disease (Broadhead, Kaplan, James \& Wagner, 1983; Case, Moss, Case, McDermott \& Elberly, 1992) .

It is beyond the scope of this dissertation to go into more detail regarding the possible consequences of the different modes of anger expression on cardiac health. It is merely pointed out that the issue is still unresolved although the finding that seems to be most consistent is that frequently expressing anger outwardly is more damaging to the cardiac system (Mendes de Leon 1992, Siegrnan \& Snow, 1997).

Social support, on the other hand, showed no associations with Type A. However, it was significantly related, negatively, with anger but only when the work supervisor informed about the characteristics of the participant. The fact that social support was negatively related to anger expression only in the work place and not at home further supports the notion about the influence of situational characteristics on psychosocial factors that may be related to health. Those who show anger manifestations at work are not perceived as inclined to make use of social resources whereas at home the spouse does not report this negative association. It seems possible that in their role as employee people may hold their anger (at work) and display more social support related behaviours because it is more acceptable or appropriate in that social context. At home, probably there are less such restrictions. More research is needed comparing presence of psychosocial risk and protective factors in different environments. In addition, the emergence of social support as a variable closely associated with elevated blood pressure indicates the need to further explore the fole of protective factors in relation to cardiovascular health.

The importance of social support as a psychosocial factor related to cardiac health is emphasised by previous findings where it has been shown that it is a mediator in the relationship between Type A and cardiac risk (House, Robins \& Metzner, 1982), that lack of social support is a good predictor of CHD mortality (Orth-Gomér, Rosengren \& Wilhelmsen,1993), that sccial support reduces cardiovascular reactivity to stress situations (Uchino, \& Garvey, 1997), and that it is related, in an inverse manner, to ambulatory systolic blood pressure in women (Linden, Chambers, Maurice \& Lenz, 1993). Moreover, it has been found that, in patients with congestive heart failure, family social support predicts lower mortality among males and having a confidant is protective of mortality in females (Coyne, Cranford, Sonnega, \& Nicklas (1998). The association between Type A and anger 
expression has also been shown to be mediated by social support in female mature university students in New Zealand (Spicer \& Hong, 1991).

In summary, the results of the present dissertation suggest that the association of Type $\mathrm{A}$ and anger is stable across different environments, that anger-out seems to be a more important component of the Type A composite for men than for women, and that social support is not related to Type A at home or at work but to anger in the working environment. Further interpretations of these results should bear in mind the possible influence of cultural factors as the studies reported above were conducted in two dissimilar cultural settings (Chapter 3 in England and Chapter 4 in Venezuela). The possibility exists that the patterns of associations among psychosocial factors are modulated by intrinsic social relationships and norms particular to each society or region in the world.

\subsubsection{Gender differences in Type $A$ and anger expression}

The most salient finding in our studies regarding Type $\mathrm{A}$ and gender is that in Venezuela more women than men were Type A. Contrasting results were found in the British sample of university employees. (Chapters 3 and 4). At a qualitative level, results in the study also from Venezuela described in Chapter 2, showed first the disproportionate distribution of women and men in top managerial levels in a highly prestigious oil-company and second the high proportion of Type A women among that group (5 Type As to 1 Type B). Taken together, these results may indicate that, in this cultural context, women are at a disadvantage concerning psychosocial risk factors for CHD.

Type A in women, nevertheless, was more related to time pressure and impatience and less to anger. This may be somehow related to the multiple roles and responsibilities requiring women to rush from one obligation to the next and to do two or more things at the same time and may indicate gender differences in composition of the TABP. Type A behaviour in women may also reflect the need to excel in their role as workers and to have higher job satisfaction. In fact for women it has been found that in Australia having multiple roles was related to higher job satisfaction. Moreover, managerial and professional women reported that the wife role was the most stressful one and that the parental role was related to exhaustion and to frequent headaches, smoking and drinking (Langan-Fox \& Poole, 1995). Thus, the costs of job satisfaction in women may be quite high in terms of CHD risk factors.

Previous results, regarding gender differences in Type $A$ and related variables are ambiguous. For instance, in a study comparing house-wives, working-women and working- 
men, Weidner, et al., (1997) found that working women had lower levels of hostility and reported fewer illnesses than house-wives and had lower cholesterol levels than housewives and than employed men. But when comparing only working women and men it was found they were quite similar in levels of hostility and symptoms reporting. In fact, men had in general more biological risk factors than women. Finally, and opposite to our findings, there were no Type A (JAS) differences between working men and working women or housewives. Cross-cultural differences may account for these results and point to the need to assess Type A specifically for each group, either men or women, and take into consideration different age, educational or occupational levels.

Friedman et al.'s (1996) argument about variations in Type A in different groups of women (e.g. from developing and industrialised countries), seems to hold according to our findings. In Caracas there were more Type A women than Type A men, opposite to what has been found in the USA. When the Type A prevalence found in women in the study described in Chapter 4 (university employees, $67.7 \%$ ) is compared to the prevalence in the Micale (1992) study (middle managers, 53\%), then one would say that even in women with different occupational levels within the same culture there will also be variations. In fact, these differences simply replicate those found in men.

Results regarding gender differences in anger expression style were also of interest. In men, outward expression of anger was a more prominent characteristic of their Type A status, whereas in women no Type A-Type B differences in anger expression styles were found. Previous investigations have found that there were no differences between college men and women in "anger-in" or "anger-out" assessed by the Framingham angerscales. (Thomas, 1989; Thomas \& Williams, 1991).

In the study reported in Chapter 4, it was found that in men "anger-out" was associated with several adverse health indicators whereas in women this was the case for "anger-in". Acknowledging the limitations of self-reported health indicators, this finding would suggest that showing anger outwardly is not as damaging for women as it may be for men, and that the same argument could be applied to men with respect to anger-in. It is, however, not suggested here that "anger-out" is better for women and that "anger-in" is recommendable for men. The results obtained here merely suggest that there may be gender differences in the consequences of anger expression styles upon health.

In a recent conference entitled "Women's hearts differ from Men's", Matthews (1998) pointed out that women's symptoms are more often attributed to psychosomatic or psychiatric causes and, thus, receive less attention from cardiologists, thereby benefiting less from possible prevention programs. Matthews also pointed out, that in the USA women may 
have more socio-demographic risk indicators such as higher unemployment, lower income, lower education, and caring for a household with children on their awn. Moreover, there is a higher percentage of women widowers than men. In agreement with our findings she reported that most of the studies point out to women tend to keep their angry feelings and their hostility to themselves. Thus, in general, CHD risk factors are different for men than for women and there is a strong need for gender-specific models. Our results from relatively small samples support this notion.

\subsection{VITAL EXHAUSTION.}

\subsubsection{Construct Validity and Pattern of associations}

In this first approach to study Vital Exhaustion in a Latin American culture, it was found that also in this context it is an internally consistent construct, and that it shares many similarities with VE as it has, been described in The Netherlands (Meesters \& Appels, $1996 \mathrm{a}, \mathrm{b})$. The reliability of both measuring instruments, the questionnaire (VEQ) and the interview (VEI), was good. This is an encouraging result in terms of the theoretical endorsement for the Vital Exhaustion construct. The successful replication of its unidimensional structure in a very different culture indicates that VE is most likely a universal concept in which enhanced irritability and indicators of lack of energy and demoralisation constitute key features.

Two items did not show sufficient item-total correlation and were removed on statistical grounds. These items were about "disrupted sleep" and "shrinking from work" " This is in disagreement with the Dutch version especially with regard to the sleep disturbances, item, which is considered a core component of VE. A hypothesis about possible cultural differences in sleep patterns in the general population due to geographic factors was proposed in Chapter 5. Further research is needed in order to test this hypothesis. So far, our results indicate that sleep problems may not be so much part of the VE construct as they are in The Netherlands. A comparison of sleep habits and disturbances between the two cultures would provide valuable information in this respect.

\subsubsection{Discriminative power of Vital Exhaustion}

Vital Exhaustion proved to discriminate, with even stronger power than in The Netherlands, between first AMI patients and healthy controls (chapter 6). This was true after controlling for well-known risk factors such as age, smoking, exercise and, to a lesser extent, 
level of education. Moreover, a close examination of the differences between separate items showed that cases had a higher percentage of positive responses than controls on all items, reaching significance in all but six of them. These results indicate that also in Venezuela, VE may be an important risk factor for AMI as was previously found in The Netherlands. The mean V'EI value for AMI cases found in the study reported here is very similar to that found in previous Dutch studies (Venezuela: 7.9(5.6); Meesters \& Appels, (1996a,b): 7.8(6.0) whereas those of the controls were lower in the Latin American sample (Venezuela: 2.2 (2.4); Meesters \& Appels, (1996a,b): 3.6(4.0)). Although we do not intend to discuss these figures as a straight comparison, they seem to indicate that the large odd' $s$ ratio found in Venezuela might be caused by the very lower level of exhaustion of the control group rather than on higher levels of exhaustion in the AMI cases in the two cultures. The reasons why the control group reported such low levels of exhaustion may have to do with social desirability within the work environment. The positive associations found with age and education point to the need to evaluate VE in different age groups and educational levels, as its status as a risk factor may be dependent on those important sociodemographic indicators.

The very high odd's ratio found in Venezuela is a matter of some concern. In fact, this is the largest OR reported in all published VE studies. Educational level was one of the aspects that may have had some influence. In addition, some methodological limitations could have affected the results. For instance, the fact that AMI patients were asked retrospectively about their exhaustion symptoms does not ensure that their responses were not affected by their feelings and symptoms at the time of questioning or, indeed, by the need to give meaning to their AMI. It is now well documented that a large proportion of patients attribute their heart attacks to psychological rather than physical motives (Willich , Lewis, Lowel , Arntz, Schubert., \& Schroder (1993). Moreover, as pointed out by Meesters \& Appels (1996b) VE thefore the AMI may in fact have been reflecting already existing sub-clinical heart disease and may thus not be considered as a predictor of cardiac disease but just as a marker of it. However, results by Kop et al., (1996) suggest that VE is not at all associated with left ventricular ejection fraction and marginally at most $(\mathrm{p}=15)$ to the extent of coronary atherosclerosis. Yet, the possibility exists that other sub-clinical disease indicators apart from the two mentioned above may cause the exhaustion symptoms prior to the AMI (Meesters \& Appels 1996b)

We may conclude that, in spite of the possible methodological limitations of both our studies, a state of Vital Exhaustion is most probably also a predictor of a first AMI in a Latin American context and that it is worth while pursuing further investigations so that a clearer picture about the role of exhaustion and negative emotions that seem to precede the onset of 
an AMI can be drawn. Subsequent prospective research should be conducted to confirm our findings, thereby overcoming the methodological limitations of case-control studies.

\subsubsection{Vital Exhaustion, Type A and Anger Expression}

With regard to the patterns of associations between VE and other psychosocial risk factors for CHD, it was found that those with Type A behaviour, those with inadequate anger expression styles, and those who perceived less social support from their surroundings were more exhausted than others (Chapter 5). Nevertheless, in the study in which AMI cases and healthy controls were compared (Chapter 6), VE was only associated with "anger-in" but not with Type A, "anger-out", or social support. Anger-control, on the other hand, was inversely related to exhaustion, but only in the AMI cases. This finding was somewhat surprising as the control group in this later study was drawn from the previous one (Chapter 5) in which the reliability and validity of the VE instruments were assessed. This small sample was selected to match the AMI cases group who were somehow older and less educated. Thus, results with the control group differ with those of the total sample. Age differences and level of education may, therefore, account for these results. In the younger groups fatigue could be more related to daily hassles and job concerns which may result in enhanced irritability feelings and perhaps more difficulty to perceive social support. The relationship between VE and different types of stressors should thus be further explored in different age groups.

It is interesting to note that AMI patients and conirols differed only on "anger-in" but not on "anger-out" or "anger-control". In previous studies in the Netherlands it was found that several dimensions of hostility clearly differentiated cardiac patients from healthy and hospital controls but also that this association was stronger in younger people. Meesters \& Appels $(1996 \mathrm{a}, \mathrm{b})$ found an interaction between total Ho scores and age, in that hostility as associated with increased risk of AMI only in patients under 50. The Buss-Durkey Hostility Inventory (BDHI) on the other hand indicated that only the Resentment and Suspicion subscales were significantly associated with elevated risk of AMI. These represent the experiential aspects of hostility as opposed to the Expressive Hostility sub-scale of the BDHI. Somehow, these two dimensions could be compared to the "anger-in" and "anger-out" scales used in our studies. They indicate that in Venezuela as well as in the Netherlands it is the tendency to withhold irritability, and not so much the outward expression of angry feelings that may be the more damaging for cardiac health. 


\subsubsection{Vital Exhaustion and Negative Mood States}

The issue of the similarities between VE and other negative mood states was approached in Chapter 5 by the use of a negative self-concept scale that included items referring to low self-esteem and self-blaming, which are related to a depressed state. In this sample of healthy participants, we found that those with exhaustion scores above the median reported having significantly more thoughts that were related to low-self esteem than those scoring below the median. In the following study, comparing AMI cases and controls, VE was related to a negative self-image but only in the AMI cases but not in the controls. It seems plausible to argue that it is particularly the synergistic combination of negative self-image and exhaustion that constitutes a elevated risk for AMI. Naturally, this hypothesis should be tested in future studies looking specifically at the interactions between VE and negative mood states in healthy individuals and cardiac patients.

In a previous report was commented that mood disturbances such as guilt feelings and low self-esteem are mostly absent in exhausted individuals (Appels, 1997). These comments were based on a vast clinical experience with patients in the Dutch studies but, as yet, there are no studies in which VE and depression have been assessed in AMI cases and controls. There exists, however, a study in which the distinctions between VE and depressed mood in a non-clinical sample was made clear. van Diest \& Appels (1991) found that whereas tiredness and fatigue distinguished between exhausted and non-exhausted participants, depressed mood assessed by means of the Profile of Moods State (POMS) and the Beck Depression Inventory (BDI), and independently by two psychiatrists, was not reported by the exhausted individuals, nor diagnosed as such by the psychiatrists. From these intriguing results the authors conclude that the shared variance between VE and depressive symptoms had more to do with the lack of energy and enhanced irritability aspects than with the low self-esteem or guilt feelings typical of depression.

Our present results seem to be somewhat opposed to those findings as the exhausted individuals did have thoughts about a poor self-image. Cross-cultural differences in attributional systems, or even in linguistic content (Appels, personal communication) may have been at the root of these discrepancies. It seems possible that in the sample studied in Venezuela, there was a tendency to attribute feelings of tiredness and lack of energy more to personal failure than has been found in other cultures, specifically The Netherlands. In their initial search for meaning, exhausted individuals may look for both internal and external causes for their symptoms and feelings. There could be cultural tendencies, even influenced by aspects such as rnethods of upbringing and religion, about 
whom to blame when one experiences such feelings as excess fatigue or demoralisation. The check whether our observations are really opposed to the findings by Van Diest \& Appels (1991) we re-run the logistic regression analysis controlling for poor self-image. Results showed that the odd ratio for VE did not decrease and that poor self-image did not discriminate between cases and controls. Therefore, the discriminating power of VE is independent and not apparently due to the fact that patients had a low self-esteem image.

Our findings are by no means conclusive. It must be acknowledged that the use of only a five-item scale may have been too restricted to assess the complex nature of negative self-image. In future studies the use of sub-scales tapping more extensively into very specific aspects of self-image is recommended. Moreover, it would be of great interest to ask patients about their perceptions of the causes of their AMI, as well as about their attributions regarding their feelings in the months previous to the event.

Thus, it is important to continue investigating this issue in order to define the boundaries of the negative mood state that seems to precede AMI. In a growing number of studies, Vital exhaustion has shown to clearly discriminate between AMI cases and controls. Nevertheless, there are controversial results in the recent literature concerning depression. For instance, Freedland, Camey, Davila-Roman, Rich, Skala, \& Jaffe (1998) report that psychiatric depression was related to congestive heart failure whereas Shapiro, DePena, Lidagoster, Woodring, Pierce, \& Glassman (1998) found that there were no associations of major depression, as assessed by means of the Diagnostic Interview Schedule and the Beck Depression Inventory, with cardiac mortality or with increased risk in $\mathrm{CABG}$ surgery patients six months after the operation. These controversial findings may as well derive from conceptual differences as from the use of distinct measuring instruments. Besides, the objectives of these studies also differed, with some referring to symptoms before the AMI and others to those that may arise as a consequence of it. Therefore, it is essential in future research to very clearly define, and separately assess, the various aspects or symptoms that correspond to a depressive-like state or to vital exhaustion.

\subsubsection{Vital Exhaustion and Social Support}

In relation to socjal support, which has not been studied previously in relation to VE, findings showed that it was inversely associated with the Interview scores in a healthy sample (Chapter 5). This seems to fit the image of an individual who has become tired and irritable and who perceives that those around him are not of much help to him to improve 
his mood and physical symptoms. However, this relationship was not found either in AMI cases or in the sub-sample of healthy controls (Chapter 6). Again, the issues of age and level of education may account for these findings, as these were the aspects that differed in both our studies. In older people it may be thought that exhaustion symptoms are more related to physical complaints and possibly to some deterioration of the organism, thus the person may not look so much into the social surroundings to explain the causes of his/her malaise.

\subsubsection{Gender differences in Vital Exhaustion}

Women scored higher on VE than men when evaluated with the VEI (Chapter 5). This result, taken together with those concerning Type A in women, may indicate that, in this cultural context, women are at a disadvantage concerning psychosocial risk factors for CHD.

These results should be interpreted with caution, nevertheless, as VE in healthy women may not be so much more related to MI risk but rather to multiple roles and daily responsibilities. Women may also be more exhausted because of their Type A behaviours that particularly relate to time pressure and impatience. Whether this combination is anticipatory of CHD in them is a matter of prospective analysis in future research. It seems plausible that this greater exhaustion in women could partially account for the increase in CHD morbidity and mortality in the female population in the past few years. In fact, previous findings indicate that VE is also a risk factor for CHD (Appels, Falger \& Shouten, 1993). Another important finding in relation to gender differences was the association of VE with several poor - self-reported - health indicators in men but not in women. This is most relevant in terms of the consequences of fatigue and exhaustion for men and women. Seemingly, men who had experienced VE symptoms in the past few months had reported higher work absenteeism due to health reasons and also reported more days in bed. In women there were no associations of VE with health indicators. It would be premature to draw any conclusions at this early stage and we may simply suggest that, given these preliminary results, it is worth pursuing further research into both the consequences of exhaustion symptoms in men and women and the probable gender differences in the aetiology and meaning of the fatigue symptoms. 


\subsection{SOCIODEMOGRAPHIC ISSUES IN PSYCHOSOCIAL RISK FOR AMI}

Throughout this general discussion, sociodemographic aspects such as occupational level, gender, age and culture, have been considered in relation to the two main variables of study. Type A and VE, and also with regard to anger expression styles. Likewise, the need to interpret results in the light of specific characteristics of the participants has been emphasised. In this section we will pay brief attention to findings regarding marital status and educational level in relation to Type A and VE.

Having or not having a partner was unrelated to either Type $A$ or to VE in all of the studies here reported. It has previously been stated that being married constitutes a protective factor for health (Gove, 1973). Moreover, in the RCSS, a large prospective study, Mendes de Leon \& Meesters (1992) it was found that never-married men had the highest risk for all-cause and coronary mortality. Our results seem to indicate that marital status, both in the British and Venezuelan groups, was unrelated to psychosocial risk factors. Thus, the association of marital status with cardiac disease does not seem to be mediated here by Type A characteristics or fatigue states. It is difficult at this stage to interpret this controversial result, which may have been due to the fact that data from those married and cohabiting was compressed and that no differences were made between those who had no partner at present because they were single, divorced, separated, or widowed.

\subsection{METHODOLOGICAL ISSUES}

\subsubsection{Questionnaires and Interviews}

That Interviews are the more appropriate method for data collection in psychosocial risk factor research than questionnaires seems to be an accepted fact. Limitations of questionnaires such as self-presentation bias, especially in the case of negative mood states, have been acknowledged (Barefoot, 1992). Nevertheless, the advantages of questionnaires in terms of time saving factors and easiness of data collection have repeatedly been enumerated (Meesters \& Appels 1996a,b). These are mostly practical arguments that should not deserve more weight than scientific methodology requirements or the need for accurate and reliable information. Interviews, on the other hand, offer the possibility of checking the participant's understanding and interpretation of questions and of making sure that all items have been addressed. 
Our Type $A$ and VE findings are very clear examples of this latter argument. In both cases the interviews were shown to have greater predictive and discriminative power as well as showing stronger associations with other risk factors. Questionnaires may serve the purpose of first level data collection or of clinical application within the physician's consulting room, but interviews should follow when there is predictive or discriminative interest. Research time will increase but much firmer conclusions will probably be drawn from it.

Yet, interviews are not free from criticism either and they do have important shortcomings, the most important ones being subjective bias by interviewers and their, unavoidable, awareness of the cardiac status of patients in the case of the VE case-control study. However, these two possible limitations were overcome by using very specific interviewing instructions and highly trained interviewers who were unaware of the hypotheses under investigation.

Thus, it may be concluded that interviews seem to be the most advisable method of data collection in this field at least for research purposes. At a clinical level, where time and expertise in interviewing may be scarce, the questionnaire is the type of instrument to use for a first screening. Otherwise, future research should focus on developing shorter versions of the Type A and VE interviews that retain similar psychometric properties in order to avoid the possibility of false positives, which seem the major weakness of questionnaires.

\subsubsection{The use of other informants in psychosocial risk factors research}

The environment and the challenges it poses seem to be of great importance according to our results. Supervisors and spouses did agree about the presence of Type A, anger, and social support related-behaviours in the participants but at a low significance level whereas spouses and self-reports did match with a high level of significance (Chapter 3). It is acknowledged that spouses may have more opportunities to observe their partners but then the challenges in the two environments are rather different and both informants have relevant and unique information to convey. According to our results that information barely agreed.

Spouses' reports did serve the purpose of corroborating the associations of selfreported anger expression styles and the VE Interview scores (Chapter 5). This was, the case with "anger-control" and "anger-in", where both self-reports and spouse-reports were related to exhaustion scores. It is interesting to note that spouse-reported "anger-out", probably the most easily observable form of anger expression for spouses, was not related to VE interview 
scores but only to the questionnaire scores. Further analysis should be carried out to evaluate the level of disagreement between the informants and its association with exhaustion.

Of particular relevance to our results is a recent report by Siegman, Townsend, Blumenthal, Sorkin \& Civelek (1998), in which spouses were asked about the anger manifestations of the study participants (thallium stress test-undergoing male and female coronary patients) by using the Spielberger's State-Trait Anger Ex.pression Inventory (STAXI). The main purposes of the study were to see the role of "anger control" in CHD risk, the combined role of anger-trait and anger-expression, and to test the hypothesis that "angerin" is a risk factor for CHD in women but not in men. Their results showed that men and women did not differ in any of the anger measures. with the exception of spouse reported Angry Reaction scores in women. "Anger-out" self-ratings cornelated with CHD whereas spouses-ratings did not. Spouse "anger-out" scores correlated with CHD when they were part of a factor that included a low threshold to react angrily only in males. There was also a significant inverse relationship between self-reported and spouse reported "anger-control" and CHD but then again, only in men. Thus, the CHD risk of anger-in in women was not confirmed. The relationship of anger-out with CHD risk is, however, in agreement with previous findings by Siegman \& Snow (1997). What seems most relevant from these study results is first that, in agreement with our results, the relationship between anger expression styles and CHD may gender specific. Second, spouse ratings of emotional expression and behaviours in patients, may be even more valid than patient's self-reports when CHD risk is concerned.

Thus, the type of environment should be included in the conceptualisation of Type A and related constructs and more research should be conducted to i) evaluate differences between those with a more generalised Type $\mathrm{A}$ behaviour and those who respond only in a Type A manner to specific challenges or environments, 2) as in 1) to know more, about the specificity of anger expression styles and social support behaviours, 3) to use other informants such as peers at work (including supervisors and subordinates) and 4) to examine in more detail the level of disagreement between informants and its relationship with cardiac disease end-points such as AMI, angina or level of obstruction of coronary arteries.

\subsubsection{Methodological limitations}

The limitations of case-control studies have already been discussed in Chapter 6. The retrospective nature of this method is a matter of concern due to both the possibility of recall bias and the fact that already diseased patients were recruited. In spite of the criticisms of 
cross-sectional studies, these continue to be one of the most frequently used methods of research in behavioral medicine. The studies reported here are no exceptions. Appels, Kop, Meesters, Markusse, Golombeck \& Falger (1994) very clearly argue that, when trying to assess the discriminative power of a purported risk factor, information gathered retrospectively in AMI patients may be biased by memory failures in the recollection of feelings and events before their AMI, and by search for meaning after having gone through a most traumatic experience. Appels, et al (1994) quote a report of a study by de Vos (unpublished) where spouses were asked to independently rate the level of exhaustion of their partners in the months preceding their AMI. Their mean score were almost identical to that of the patients and the correlation was .86 , probably indicating that the reports by the patients were reliable. The possibility also exists, however, that spouses were going through a traumatic process as well and were also searching for meaning to what had just occurred to their partners. In another study by Trijsburg, Erdman, Duivenvoorden, Thiel, et al., (1987) it was found that AMI patients rated their exhaustion significantly lower than their partners did, concluding that AMI patients tended to underestimate their exhaustion symptoms before their cardiac event. Taken together these two studies give some indication that AMI patient's reports may be valid but should be interpreted cautiously.

Some comments about the experience of interviewing AMI patients at such an early stage after their AMI may be relevant at this stage. While responding to the questionnaires, patients were visibly trying to remember their feelings before the AMI and the majority gave straight forward answers while a few had doubts and required some time and further explaining of the questions

There was no sign of they responding positively to the VE items to conform to some expectation from the interviewer or to give meaning to their recent experience. Patients showed in general, good understanding of the questions and gave straight answers sometime showing that they knew very well what the interviewer was talking about. AMI patient's responses seemed to sincerely reflect their feelings and symptoms before the AMI. In the few cases were they turned into the present tense when responding the interviewer interrupted and asked again to think back before the AMI, which all patients did. These observations gave an indication that the answers did refer to the time period specified and were honest and sincere. Thus, although the possibility of recall bias is not underestimated, direct experience indicated that this may have had a minimal effect upon responses.

The use of cross-sectional designs is not due simply to the fact that results are at hand in a shorter period of time. Cross-sectional studies do have an important contribution to make. As it has been pointed out previously, four of our studies were conducted in Venezuela 
and had mainly an exploratory interest about Type A and Vital Exhaustion in this cultural context. This interest responded to the fact that psychosocial factors for CHD are very much vulnerable to social influences and the design selected here was no exception. Yet, as long as their limitations, are acknowledged, the information that cross-sectional studies provide about relationships among variables and group differences are indicative of characteristics that most likely may be related to the aetiology of the disease (Alzate, Llanos, Guerrero \& Neutra, 1990) and to risk factors associated with it (Sartwell, 1974).

The bias due to recruiting methods, on the other hand, is unavoidable in this type of study and, thus, the limitations about causal inferences must be acknowledged. We tried to avoid possible bias due to recall by conducting the interviews around 48 hours after the cardiac event when the "search for meaning" process was possibly at a very early stage and patient's information may not have been "contaminated" yet. It is advisable, nevertheless, that in future studies participants will be re-evaluated perhaps one to two months later, allowing for a comparison with the data obtained just after the AMI.

The rather small sample sizes in some of the studies here presented may be considered another limitation. Nevertheless, application of appropriate statistical analysis ensured adequate treatment of the data. In addition, our interpretation of results is always on the cautious side. In future investigations the use of larger samples is recommended.

\subsection{FINAL COMMENTS AND IMPLICATIONS FOR THE FUTURE}

\subsubsection{Type $A$, the concept: Is it alive and well?}

It is not an easy task to reduce the results of this dissertation to a few concluding remarks about Type A given the fact that it is based on distinct studies that had varied aims. In general, we may conclude that, with regard to Type A, the exploratory studies conducted in Venezuela and presented in this dissertation have positively contributed to describing the Type A Behaviour Pattern in a different cultural context, that of Latin America. Once again, Type A proved to be a stable construct. One of the most striking findings is the great influence of gender and the low influence of education or age on its presence and the associations with anger expression styles and self-reported health indicators. Its predictive value for CHD in this cultural context is another matter and will need attention in future studies.

The strong gender differences found in these studies may be due to differences in upbringing methods but also to the present demands of society for women, requiring from 
them to fulfil several roles, that is to continue with traditions (e.g. child-rearing practices), to increase family income (job role), and, most importantly, to excel in both (personal satisfaction and escalating the job ladder). Thus, it is of utmost importance to evaluate in this cultural context, prospectively, the effects of Type A and its components on cardiac health for men and women.

With regard to Type A and blood pressure the results here obtained showed that Type As did not differ from Type Bs in blood pressure measurements but, on the basis of the findings presented in Chapters 2 and 3, point to the need to consider two important issues when assessing the reactivity hypothesis as the possible pathway to CHD. First, it is important to include measures of cardiovascular activity at times other than rest and task performance, namely, when entering and when leaving the experimental session. Second, the use of other informants and their levels of disagreement in terms of the behavioural styles of the participants in different environments may also contribute to identify important risk indicators of cardiovascular functioning.

Finally, although is not the intention of the present discussion to give a full account of the history and development of the Type A concept or of its current "state of the art", some aspects related to what may be the future of Type A research deserve attention. Although Type A as a global risk factor for CHD has lost some "credibility" due to a number of negative epidemiological findings (MRFIT; Shekelle et al, 1985, Aspirin Myocardial Infarction Study; Shekelle, Gale \& Norusis, 1985) and to the findings about the allegedly stronger predictive power of hostility and anger expression styles (Barefoot, Dahlstrom, \& Williams, 1983: Williams, 1987), it may still be concluded that Type A is an important psychosocial determinant of CHD. In most of the studies conducted in different cultures, where it was evaluated prospectively by interview methods, and in participants healthy at entry, and looking not only at mortality but also at incidence and morbidity rates, Type $\mathrm{A}$ has clearly shown its negative effects on cardiac health (Friedman, Fleishmann \& Price, 1996). Moreover, its relevance in cardiac rehabilitation is shown by studies in which successful reduction of Type A and coronary recurrences was attained using cognitive and behavioral interventions (Bracke \& Thoresen, 1996: Karlberg, Krakau, \& Unden, 1998).

The inevitable question arises as to whether it is worth pursuing further research in other parts of the world about risk factors that are no longer "popular" in the Anglo-American literature. Should researchers in these other cultures follow the same path and see if similar results could be obtained? Should they put aside concepts such as global Type A and go straight to the assessment of hostility and anger as most probable risk factors for $\mathrm{CHD}$ ?. There are not straight answers to these questions. Our results show that there seem to be more 
similarities than differences between our findings and those in other parts of the world in terms of probable psychosocial risk. Naturally this would have to be corroborated in longitudinal clinical studies. We are inclined to think that psychosocial risk factors for CHD are in general universal and that differences may consist in the weight of the components or elements embedded in the various constructs. The questions of how much difference a culture can make with regard to psychosocial risk for CHD, remains open.

Finally, assessment issues are most relevant in relation to the discussion presented above. We share Friedman et all., (1996) view that Type A when evaluated using the appropriate methods (SI and Videotaped Clinical Examination, VCI), shows the closest relationship with $\mathrm{CHD}$ and with other risk factors. Type As lack awareness of their own everyday reactions and, therefore, it is necessary to assess their overt psychomotor signs which are indicative of impatience, hostility or time-pressure. Besides, hostility and anger are surely not isolated feelings but arise precisely as a result of those other most important components namely, time urgency, and insecurity and from the frustrations that inevitably follow the need for a rapid-paced life-style. Thus, time pathologies as described by Ulmer \& Schwartzburd (1996) bring about an impact on intrapersonal coherence and interpersonal relationships that may include social isolation, emotional dissatisfaction and mood disturbances including hostility and anger.

\subsubsection{Vital Exhaustion: At the end of the road in life and at the edge of a heart attack?}

Life in the year or so before a cardiac event seems to differ from previous years and this is what the concept of Vital Exhaustion has set out to outline. In retrospect, in the period prior to an MI, patients describe experiencing symptoms and feelings which are different from those of healthy individuals. This state seems to hold in different cultural contexts as it was. found in Venezuela in line with results in The Netherlands.. One could say that exhaustion is the state that may emerge when the organism is approaching the end of the road, probably after negative experiences, or inadequate coping strategies or behaviour patterns. Individuals may then reach a state which has been described as "emotional drainage" (Appels, 1980). Nevertheless, several issues are still unresolved and will merit further attention. The main ones refer first, to the theoretical foundation of the VE construct and its possible overlapping with neighbouring negative mood states, such as depression, and second, to assessment issues.

With regard to the theoretical foundation of VE it has been shown in previous studies, as well as in the ones described in this dissertation, that it is a solid and reliable. 
construct (chapters 5 and 6). Nevertheless, the question of its possible overlapping with other negative mood states such as depression or burnout is not fully resolved. Although there seems to be good evidence indicating that VE and depression are independent emotional states (van Diest \& Appels, 1991), there is an important number of studies in which depression has been found to be related to CHD (Booth-Kewley \& Friedman, 1987; Fielding, 1991, Anda et al, 1992). It has even been stated that depression is embodied within many of the items that comprise the Maastricht Questionnaire (Allan \& Scheidt, 1996) with the obvious implication of their overlapping. This issue has extensively been discussed throughout this dissertation but a few more comments could be added at this point. Van Diest \& Appels (1991) found that depressive symptoms, according to the BDI, were present to a significantly greater extent in the exhausted men. Nevertheless, clearly depressive symptoms such as loss of appetite, distorted body image, suicidal intention, or self-accusations were not at all reported by thern. In fact, the association between VE and depression was based on the items that referred to loss of vigour and elevated fatigue. Once again, the accounts of the AMI patients in our study seemed internally valid and relevant. When responding to the VE items many patients spontaneously expanded their responses in order to better describe their feelings before the AMI and in no case $(\mathrm{N}=31)$ did they express any distorted cognitions, extreme sadness, guilt-feelings or low self-esteem. No comments were made about blaming themselves for their loss of energy or difficulties to concentrate. Rather, they described in other word, the unexplainable decrease in energy, lack of motivation, not being able to perform as before and tiredness that is characteristic of Vital Exhaustion. Whatever negative mood feelings were expressed during the interview did not seem to be indicative of a clinical depression but were rather more related to their perceived inability to accomplish things as they used to.

A tentative conclusion is that VE and (clinical) depression are independent emotional states that partially share aspecis, which have primarily to do with loss of energy and fatigue. In the case of clinical depression, these latter aspects could be considered as secondary symptomatology resulting from the depressed mood, distorted cognitions and selfblaming which are its primary symptoms. In the case of exhaustion, then, fatigue, decreased energy and increased irritability are the main descriptors of the core of the construct which do not necessarily lead to the low self-esteem symptoms of depression.

As Appels (1997) pointed out, sorne authors prefer the tern "depressive symptomatology" rather than "depression" to describe AMI patients' emotional state prior to their cardiac events and this reflects well the present uncertainty about the nature and aetiology of this complex mood state. To this it could be added that the use of the term 
"depression" is perhaps misused and overused in this area and that a more precise terminology is required. In fact, this is what the concept of Vital Exhaustion is trying to convey. "Vital Exhaustion" is all about feelings of being tired, about increased irritability, and about "low spirits" but an exhausted person is not, necessarily, a "depressed" person, clinically speaking. This distinction is essential, not only for the theoretical understanding of the process, but also when discussing possible interventions.

Vital Exhaustion also shares some common elements with the concept of "burnout" described as "a syndrome of emotional exhaustion, depersonalisation, and reduced personal accomplishment" (Maslach, 1982). Although this concept has been mainly described in individuals who deliver people-related services (e.g. teachers, nurses) it could obviously be extended to describe individuals in any other type of work settings. The Maslach Bumout Inventory (Maslach \& Jackson, 1986) includes items such as "I feel emotionally drained from my work", I feel used up at the end of the work day" which are very similar to some of the items in the VE instruments that relate to work. Nevertheless, it may be said that vital exhaustion encompasses a wider range of symptoms beyond work and, more importantly, that it seems to describe the final stages of burnout: "prolonged fatigue, insomnia, loss of libido" inability to concentrate, and feelings that one's life is out of control" (Stanton-Rich \& IsoAhola, 1998)

Several issues regarding the discriminative power of VE in our studies should be addressed at this stage. Why was the OR so high in Venezuela compared to that in previous studies in The Netherlands? Besides the already acknowledged methodological limitations and the existing effect of educational level, the proportion of exhaustion in the AMI patients compared to the healthy controls was unexpectedly high. In fact, it was twice as high as that found in previous studies ( $O R=8.11$. Meesters \& Appels, 1996b; OR=7.35 neighbourhood controls, $\mathrm{OR}=2.90$, hospital controls, Falger, 1989, $\mathrm{OR}=10.1$, Rotterdam Civil Servant Study, Appels \& Mulder, 1988; OR=2.3, women, Appels, Falger \& Schouten, 1993). Going back to the interviews with cardiac patients which took place while in their hospital beds four to five days after their AMI, it was evident that some of them were going through a difficult time after their life-threatening experience and were in the preliminary stages of irying to find a meaning to their AMI. Recent findings in the UK have shown that between $10 \%$ and $20 \%$ of AMI patients may suffer from post-traumatic stress disorder (PTSD) (Brookes \&. Bennett, 1999). It seems relevant to investigate the prevalence of PTSD in post AMI patients in Venezuela, which, due to cultural differences, may be higher accounting for possible overestimation when enquired about their pre-AMI symptoms and feelings. Naturally, this, hypothesis deserves future testing. 
Although patients are used to being approached by different medical specialists while in the hospital, it is highly unusual in Venezuela that someone will approach AMI patients, or any other type of patient, to primarily inquire about feelings, emotions and cognitions while in bed at the hospital. Although the hospital in which the patients were recruited is one of the few in the country to run a cardiac rehabilitation program which starts as soon as they are discharged from the coronary care unit, the program at this stage consist only of a visit form the physiotherapist and the cardiologist to explain the program and the possibility of joining it after discharge from the hospital. The interview did in fact take some of the patients by surprise although none refused to answer the questions. The possibility exists that inquiring about personal feelings at a moment still so close to the AMI, a life threatening experience for which they found no apparent reason, may have given the patients some hints and understanding about why this had happened to them and, although patients gave a clear impression of sincerity, again it may have been possible that accounts were slightly disproportionate with respect to their pre-AMI feelings.

In contrast, in the healthy controls social desirability may have worked the other way round in reducing the possibility of reporting symptoms that were undesirable, particularly when reported within the work environment where the data was collected. Due to this effect, and to the aspects related to a possible adaptation to daily hassles mentioned earlier, healthy controls may have concealed some of the symptoms in order to avoid a negative image. Although they were reassured that the evaluation was absolutely confidential and that it had nothing to do with promotion or else. susceptible participants may have not been fully convinced by this information. Therefore it is quite possible that the high OR of VE observed in Venezuela should be partially attributed to the low level of VE in the control group

If it is accepted that prior to an AMI individuals go through a period of tiredness, demoralization, and irritability for which there is no "apparent" reason, but which is believed to be the result of prolonged stress, several challenging questions remain, some of which have in the past received more attention than others: 1) Is VE the result of a process in the coronary arteries to the heart where psychological or psychosocial variables do not play any part?. 2) Are behaviours, emotions, and life experiences related to the process of becoming exhausted? If so, what aspects of life should it be attributed to? What is the relationship of VE with life events, job stress, unemployment, inadequate coping strategies, minor life-events or behaviour patterns? In this respect, an interaction effect between biological and psychosocial factors seems most likely and future research should focus on the possibility and nature of this interaction. 
Research into the possibility that VE is the result of sub-clinical heart disease is currently going on and preliminary results appear to be promising. The Maastricht group is presently conducting a series of investigations testing the hypothesis of whether VE is a marker of functional processes in the coronary vessel wall in angioplasty patients. In preliminary studies they observed a positive association between VE and interleukine-1 in these patients. Those observations led them to consider the possibility that the strong association between VE and short-term CHD might be partly based upon the functional status of the vessel wall. Preliminary data suggested a two-stage model in which VE as precursor of CHD, is part of a circular process: 1) Prolonged stress may result in feelings of tiredness and a decrease of immunocompetence. 2) Decrease of immunocompetence may result in an activation of latent viruses, leading to the inflammation of coronary vessels. Due to the release of cytokines, the feelings of tiredness increase and may result in a state of VE. Thus, there is no "either - or" question (i.e. is VE a marker of the disease or an independent psychological risk factor), but rather a question of how a specific mental state and the inflammation processes may reinforce each other. This model was supported by recent findings (Appels, 1999). Blood samples of 15 exhausted and 15 non-exhausted angioplasty patients were compared and biopsies from their culprit lesions were also taken. Findings showed that the exhausted patients, according to the VE interview, had higher levels of the cytokines IL $1 \beta$ and TNT $-\alpha$, and tended to have higher levels of IL-6. These results indicate that exhaustion and cytokine levels are strongly associated in angioplasty patients. The author acknowledges the need for future prospective studies to ascertain the direction of the association. This is an. interesting line of research which, if the model is further supported, would give weight to the notion of interaction between biological and psychosocial processes as a risk for CHD. Methodology permitting, studies examining the relationship of cytokines with other cardiac end-points should be conducted in the future.

Concerning the specific conditions or reasons for the emergence of exhaustion in some individuals, this is also an issue that has to be carefully addressed in the future. In particular, the issue of the relation of job characteristics and unemployment with VE is relevant at this point. Falger \& Schouten (1992) found a strong association between VE and prolonged overtime in male cardiac patients. In women, those who had multiple roles, that is working outside the home and simultaneously taking care of the household and children had significantly higher scores on the MQ than women without housebold responsibilities.. Thus, amount of work as well as quality of working conditions seem to be clearly associated with fatigue in both men and women. 
Time pathologies are also very much related to work and need for achievement and may lead to exhaustion. Based on their vast clinical experience Ulmer \& Schwartzburd (1996) concluded that for their time-urgent cardiac patients, exhaustion seemed to be a risk factor for CHD rather than it being the result of worsening disease process. People who suffer from severe forms of time pathologies often report sleep disturbances, fatigue, abnormal breathing patterns, enhanced irritability, and depressed mood. Future research should focus on the relationship between job characteristics and time pathologies, as well as on the consequences of prolonged periods of unemployment, and exhaustion. There is no reason to believe that there is only one single pathway to exhaustion. The possibility exists that there are distinct reasons or causes for it, some more related to emotional drainage due to physical deterioration of the organism (e.g. overwork, multiple roles) and others (like unemployment, negative life events, daily hassles) that are also due to emotional drainage but perhaps more related to the inability to accomplish a purpose in life accompanied by low self-esteem. In any case, individuals may reach a state in which they lack personal or physical resources to cope with the excessive stress in their lives. Another related issue that needs further exploring is that of major life events in relation to VE. The intense and/or frequent encounter of stressful experiences, which has been generally associated with cardiac disease, may be mediated by a state of emotional drainage, lack of energy and fatigue, that is, Vital Exhaustion, when one gets to the end of the road.

Second, with regard to the assessment of Vital Exhaustion, we concluded in previous sections that the Interview is the most appropriate method to capture the truly exhausted individuals, while avoiding the possibility of false negatives. Nevertheless, this a costly method in terms of time and trained staff and, therefore, efforts should be devoted to the improvement of the questionnaire format perhaps by being more explicit and specific as to the meaning of each item. One way of achieving this aim may be by adding some of the clarifying questions that are part of the interview to the questionnaire (e.g. item 4: "were you irritated more easily lately than before"? adding to the same item: "Did somebody (wife, children, friends at work) tell you that you have become more irritable lately?). Another possibility to ensure that the syrnptom was present within the specified time limit is adding two columns in the response scale, one saying "Yes, 1 have been like this for a long time, (most of my life)" and the other "Yes, I have been increasingly become like this in the past 10 months or so". In this way the respondent would have a more specific time reference to dellimit the presence or absence of each exhaustion symptom.

To finally conclude, results from these investigations point to a more or less. universal pattern of psychosocial risk factors for CHD. Nevertheless, recognition of local, 
regional or cultural variations in the entire construct or elements, as well as in their particular associations; with other constructs or risk factors, is of vital importance at all stages of study from the theoretical one to their application in prevention and rehabilitation. Type A and Vital Exhaustion evaluated in a Latin American context showed a reasonable stability that was influenced, mainly, by gender. The most important contribution of these studies may be in terms of prevention. The diagnosis of Vital Exhaustion in patients that show other biological (dislipidemia, obesity, smoking on family history) or psychosocial (Type A, anger, hostility, time pathologies) cardiac risk factors, may indicate the proximity of a major cardiac event. A second, but no less important contribution, is in terms of intervention programs in exhausted individuals both previous: and after an AMI. Cognitive-behavioral therapies oriented at changes in work attitudes and life-style, time-management, and restructuring of personal aims may help individuals to avoid reaching an state of mental and physical exhaustion. 


\section{REFERENCES}

Abbot, J., \& Sutherland, C., (1991) Cognitive, cardiovascular and haematological responses of Type $A$ and Type $B$ individuals prior to and following examinations. In Strube, M.J. (Ed.) Type A Behavior Sage Publications, Newbury Park, London (pp. 313-338).

Agras, S. (1982). Behavioral medicine in the 1980's. Journal of Consulting \& Clinical Psychology, 50, 7917-803.

Allan, R. (1996) 'The emergence of Cardiac Psychology. In: R. Allan \& S. Scheidt (Eds.) Hearl \& Mind. The Pracice of Cardiac Psychology. APA, Washington, DC (pp. 3-14)

Allan R., \& Scheidt, S. (1996) Empirical Basis, for Cardiac Psychology. In: R. Allan \& S. Scheidt (Eds.) Heart \& Mind. The Practice of Cardiac Psychology. APA, Washington, DC (pp. 63-124).

Alzate, A., Llanos, G., Guerrero, R. \& Neutra, R. (1990) Estudios Epidemiológicos. Modulos de Epidemiología. Caracas.

Anda, R., Williamson, D., James, D., Macera, C., Eaker, E., Glassman, A., \& Marks, J. (1992) Depressed affect, hopelessness, and the risk of ischemic heart disease in a (1993) cohort of US adults. Epidemiology, 4, 285-294.

Appels, A. (1980) Psychological Prodromata of myccardial infarction and sudden death .Psychotherapy and Psychosomatics, 34, 187-195.

Appels, A. (1983) The year before myocardial infarction . In: T.H. Dembroski, T.H. Smith, \& G. Blumchen (Eds.) Biobehavioral Basis of Coronary Heart Disease. Carger, New York (pp. 18-38)

Appels. A. Hoppener, P.. \& Mulder, P., (1987) A questionnaire to assess premonitory symptoms of myocardial infarction. International Journal of Cardiology, 17, 15.24.

Appels, A. \& Mulder, P. (1988). Excess fatigue as a precursor of myocardial infarction. European Heart Journal, 9. 758ิ-764.

Appels, A. \& Mulder, P. (1989) Fatigue and heart disease. The association between vital exhaustion" and past, present, and future coronary heart disease. Journal of Psychosomatic Disease, 33, 727-738.

Appels A. (1990). Mental precursors of myocardial infaretion. British Journal of Psychiatry, 1156, 465-471.

Appels" A. (1997) Depression and coronary heart disease: Observations and questions. Journal of Psychosomatic Research, 43, 443-452. 
Appels, A. \& Otten, F. (1992). Exchaustion as a precursor of cardiac death. British Journal of Medical Psychology, 31, 351-356.

Appels, A., Falger, P.R.J., \& Schouten, E. (1993). Vital exhaustion as risk indicator for myocardial infarction in women. Journal of Psychosomatic Research, 37, 881-890.

Appels, A., Kop, W., Meesters, C.,Markusse, R., Golombeck, B., \& Falger, P. (1994) Vital exhaustion and the acute coronary syndromes. International Review of Health Psychology, 3, 65-95.

Appels, A. (1998) Inflammation and the mental state before an acute coronary event Annals of Medicine, 31, 41-44.

Atchinson, M. \& Condon, J. (1994) The association between spouse-eported Type A behavior pattern and coronary heart disease. Australian and New Zealand Journal of Psychiatry 28, 298-301.

Averill, J. (1982) Anger and aggression: An essay on emotion. Springer-Verlag. New York.

Bagés, N., Warwick-Evans, L., \& Falger, P. (1996). Differences between informants about Type A, anger-expression, and social support and the relationship with blood pressure. Psychology and Health, .12, 453-465.

Bagés, N., Feldman, L. \& Chacón, G. (1991), Tipo A y reactividad cardiovascular en gerentes (Type A and cardiovascular reactivity in managers) Salud Pública de Mexico, 37(1), 47-56.

Bages, N. , Falger, P., Feldman, L., Chacón, G., Pérez, M.G. \& Guarino, L. Construct validity of Type A behavior pattern in Venezuela. Submitted.

Bagés, N., Falger, P., Pérez, M.G. \& Appels, A. (1998) Vital Exhaustion Measures and their associations with coronary heart disease risk factors in a sample of Spanishspeakers Accepted. Psychology \& Health.

Bagés, N., Falger, P., \& Appels, A. Vital exhaustion as a risk factor of myocardial infarction: A case-control study in Venezuela In press: Intemational Journal of Behavional Medicine

Baker, L., Dearborn, M., Hastings, J., \& Hamberger, K. (1984) Type A behavior in women: A Review, Health Psychology, 3, 477-497.

Barefoot, J. (1992) Developments in the measurement of hostility. In H.S. Friedman (Ed.) Hostility; Coping, and Health. APA, Washington, D.C. (pp 13-31).

Barefoot, J. \& Schroll, M. (1996). Symptoms of depression, acute myocardial infarction, and total mortality in a community sample. Circulation, 93, 1976-1980.

Barefoot,J., Dahlstrom, W., \& Williams, R. (1983) Hostility, coronary heart disease incidence and total mortality: A 25-year follow up of 255 physicians, Psychosomatic Medicine, 45, 59-63.

Barnett, R., Davidson, H., \& Marshall, N. (1991) Physical symptoms and the interplay of work and family roles. Health Psychology, 10, 94-101. 
Bennett, P. \& Carroll, D. (1990). Type A behaviors and heart disease: epidemiological and experimental foundations. Behaviorai Neurology, 3, 261-277.

Bennett, P., Gallacher, J., \& Johnston, D. (1990) Toward a state measure of Type A behavior. British Journal of Clinical Psychology, 29, 155-165.

Bienias, J., \& Strube, M., (1991) The effect of self-consciousness on Type A and Type B self.schemata. In Srtube, M.J. (Ed.) Type A Behavior Sage Publications, Newbury Park, London. (pp. 313-338).

Blumenthal, J., Barefoot, J., Burg, M., \& Williams, R. (1987) Psychological correlates of hostility among patients undergoing coronary angiography. British Journal of Medical Psychology, 60, 349-355.

Boiltwood, M., Taylor, C., Burke, M., Grogin, H., \& Giacomini, J. (1993) Anger report predicts coronary artery vasomotor response to mental stress in atherosclerotic segments. American Journal of Cardiology, 72, 1361-1365.

Booth-Kewley, S \& \& Friedman, H. (1987). Psychological predictors of heart disease. A quantitative review. Psychological Bulletin, 101, 343-362.

Bosma, H., Appels, A., \& Sturmans, F. (1995) Psychosocial factors in the aetiology of coronary heart disease: Follow-up to the Kaunas-Rotterdam Intervention Study (KRIS). Cardiology, 2, 54 59.

Bracke, P. \& Bugental, J. (1995) Existential addiction: A new conceptual model for Type A and workaholism. In T. Pauchant (Ed.) In search of meaning, Jossey-Bass, San Francisco (pp.65-93)

Bracke, P., \& Thoresen, C. (1996) Reducing Type A behavior Patterns. A structured-group approach. In: R. Allan \& S. Scheidt (Eds.) Heart \& Mind The practice of Cardiac Psychology. APA, Washington, DC, pp 255-290. 52,

Brezinca, V. \& Padmos, I. (1994), Coronary Heart Disease risk factors in women. European Heart Journal, 15, 1571.

Brezinka, V. \& Kitel, F. (1995) Psychosocial factors of coronary heart disease in women: A review. Social Science and Medicine, 42, 1351-1365.

Broadhead, W.E., Kaplan, B.H., James, S.A., \& Wagner, E.H. (1983) The epidemiological evidence for a relationship between social support and health. American Journal of Epidemiology, $167,521-537$.

Burell, G. (1996) Group psychotherapy in Project New Life: Treatment for coronary-prone behaviors for patients who have had coronary artery bypass graff surgery. In: R. Allan \& S. Scheidt (Eds.) Heart \& Mind. The practice of Cardiac Psychology. APA, Washington, DC, pp 291312.

Byrne, D., \& Reinhart, M. (1989) Work characteristics, occupational achievement and the Type A behavior pattern. Journal of Occupational Psychology, 123-134. 
Carmelli, D., Dame, A., Swan, G., \& Rosenman, R.H. (1991) Long-term changes in Type A behavior. A 27-years follow-up of the Western Collaborative Group Study. Jornal of Behavioral Medicine, 14, 593-06.

Case, R.B., Moss, A.., Case, N., McDermott, M., \& Elberly, S. (1992) Living alone after myocardial infarction: Impact on prognosis. Journal of the American Medical Association, 267, 515-519.

Catipovic-Veselica, K., Buric, D., Ilakovac, V., Amidzic, V., Kozmar, D., Durjancek, J., Skrinjaric, S. \& Catipovic, B. (1995) Association of scores for Type A behavior with age, sex, occupation, education, life needs satisfaction, smoking, and religion in 1084 employees. Psychological Reports, 77, 131-138.

Chesney, M., Eagleston, J. . \& Rosenman, R. (1981) Type A behavior: Definition, assessement and intervention. In: C. Prokop, \& L. Bradley, (Eds.) Medical Psychology: A new perspective, New York Academic Press, (pp 20-36).

Chesney, M., Hecker, M., \& Black, G. (1988) Coronary-prone components of Type A behavior in the WCGS. A new methodology, In: B.K. Houston. \& C.R. Snyder (eds). Type A behavior Pattern. Research. Theory and Intervention, John Wiley \& Sons, New York, (pp 168-188).

Chesney, M. (1985) Anger and hostility: Future implications for behavioral medicine. In: M.A. Chesney. \& R. H. Rosenman Anger and hostility in Cardiovascular and Behavioral Disorders Hemisphere Publishing Corporation, Washington (pp. 277-290).

Chesney, M., Eagleston, J., \& Rosenman, R. (1980). The Type A structured interview: a behavioral assessment in the rough. Journal of Behavioral Assessment, 2, 255-272.

Chrisman, J., Fry, T., Reeves, G., Lewis, H., \& Weinsler, R. (1989) A multiobjective linear programming methodology for public-sector tax planning. Interfaces, 19, 13-22.

Cinciripini, P. (1986) Cognitive stress and cardiovascular reactivity. Relationship to atherosclerosis, arrythmias, and cognitive control.American Heari Journal, 112, 1051-1065.

Cohen, 1., Ardjoen, R., \& Sewpersad, K. (1997) Type A Behavior as a risk factor after myocardial infarction: A review. Psychology \& Health, 15, 619-632.

Cohen, J., \& Reed, D. (1985) The Type A behavior pattern and coronary heart disease among Japanese men in Hawai. Journal of Behavioral Medicine, 8, 342-352.

Condon, J. (1988) The assessment of Type A behavior pattern: results from a spouse-report approach. Psychological Medicine, 18, 747-755.

Contrada, R., \& Krantz, D. (1988) Stress, reactivity, and Type A behavior: Current status and future directions. Annals of Behavioral Medicine, 10, 64-70.

Cooper, T., Detre, T., \& Weiss, S. (1981) Coronary-prone behavior and coronary heart disease. A critical review. Circulation, 63, 1199-1215.

Corzine, J., Buntzman, G., \& Bush, E. (1992) Relationship of Type scores to careers at plateau and job satisfaction. Psychological Reports, 71, 627-632. 
Coyne, J., Cranford, J., Sonnega, J. \& Nicklas, J. (1998) Social support as a predictor of mortality among married congestive heart failure patients. Psychosomatic Medicine, 60, 118.

Crisp, A., Queen, M., \& DeSouza, M. (1984). Myocardial infarction and the emotional climate. Lancet. 17, 616-619.

De la Casa, L., Gordillo, J., Mejías, L., Rengel, F. \& Romero, M. (1998) “Attentional strategies in Type A individuals. Personality and Individual Differences, 24, 59-69

Dembroski, T. \& Costa, P. (1987) Coronary-prone behavior: Components of the Type A pattern and hostility. Journal of Personality, 55, 2 ! 1-236.

Dembroski, T. \& Costa, P. (1988) Assessment of coronary-prone behavior: A current overview. Annals of Behavioral Medicine, 10, 60-63.

Dembroski, T., McDougall, J., Costa, P., Grandits, G. (1989) Components of hostility as predictors of sudden death and myocardial infarction in the Multiple Risk Factor Intervention Trial. Psychosomatic Medicine, 51, 514-522.

Dembroski, T. \& MacDougall, J. (1983) Behavioral and psychophysiological perspectives on coronary-prone behavior. In: T.H. Dembroski, T.H. Smith, \& G. Blumchen (Eds.) Biobehavioral Basis of Coronary Heart Disease. Carger, New York (pp. 106-129).

Denollet, J., \& De Potetr, B. (1992) Coping subtypes for men with coronary heart disease.Relationship to well-being, stress, and Type A behavior. Psychological Medicine, 22, 667-684.

Dimsdale, J.E., (1993) İnfluences of personality and stress-induced biological processes on etiology and treatment of cardiovascular diseases in women. In N.K. Wegner, L. Sperofl, \& B. Packard (Eds.) Cardiovascular Health and Disease in Women (pp 225-230). Greenwich, Cr: Le Jacq Communications.

Donker, F. (1999) Mind your heart. Risk factor education in secondary prevention of coranary heart disease. PhD Thesis. Catholic University of Nijmegen. The Netherlands.

Dunn, S.E., Putallaz, M., Sheppard, B.H., \& Lindstronin, R. (1987) Social support and adjustment in gifted adolescents. Journal of Educational Psychology, 79, 467-473.

Elliot, R. (1988) The dynamics of hypertension: An overview: Present practices, new possibilities, and new approaches. American Heart Journal, 116, 583-589.

Evans, P. (1995) Cognitive-behavioral treatment of Type A behavior pattern. A critical review. Psicologia Conductual, 3, 183-193.

Eyer, (1977) Prosperity as a cuase of death. International Journal of Health Services, 7, 125-148.

Eyer, J. (1980) Social causes of coronary heart disease. Psychotherapy and Psychosomatics, 34, 7587.

Eysenk, H.J. (1991) Type A behavior and coronary heart disease: The third stage. In Srtube, M.J. (Ed.) Type A Behavior. Sage Publications, Newbury Park, London. (pp. 313-338).

Falger, P., Schouten, E., Appels, A., \& De Vos, Y. (1988). Sleep complaints, behavioural characteristics, and vital exhaustion in myocardial infarction cases. Psychology and Health, 2, 231-258. 
Falger, P.(1989). Life-span development and myocardial infarction.: An epidemiological

Study. PhD Thesis. University of Maastricht. The Netherlands.

Falger, P. \& Schouten, E.(1992). Exhaustion, psychological stressors in the work environment, and acute myocardial infarction in adult men. Journal of Psychosomatic Research, 36, 777-786.

Falger, P. \& Nelissen-de Vos, Y. (1997) Slaapklachten als voorslopers van het hartinfarct. Gedrag \& Gezondheid, 25, 161-175.

Feldman, L. (1995) Factores psicosociales asociados a la salud de la mujer con roles multiples (Psychosocial factors assoccited with health in women with multiple roles). Unpublished manuscript. Simon Bolivar University. Section of Psychohysiology and Human Behavior. Caracas.

Fielding, R. (1991) Depression and acute myocardial infarction: A review and reinterpretation. Social Science and Medicine, 32, 1017-1027.

Fleming, R., Baum, A., Gisriel, M., \& Gatchel, R. (1982). Mediating influences of social support on stress at the Three Mile Island. Journal of Human Stress, 8, 14-22.

Fontana, A., Rosenberg, R., Burg, M., Kerns, R., \& Kolonese,K. (1991) Type A behavior and selfreferencing: Interactive risk factors? In Strube, M.J. (Ed.) Type A Behavior. Sage Publications, Newbury Park, London (pp. 215-232)

Frasure-Smith, N., Lesperance, F., \& Talajic, M. (1995) The impact of negative emotions on prognosis following myocardial infarction: Is it more than depression? Health Psychology. 14, 388-398.

Freedland, K., Carney, R., Davila-Roman, V., Rich, J., Skala, J. \& Jaffe, A.S (1998) Major depression and survival in congestive heart failure. Psychosomatic Medicine, 60, 92-135

Friedman, H. \& Powell, L. (1984) The diagnosis and quantitative assessment of Type A behavior: Introduction and description of the Videotaped Structured Interview. Integrative Psychiatry, 2, 123-136.

Friedman, H. (1989) The role of emotional expression in coronary heart disease. In: A.W. Siegman \& T.M. Dembroski (Eds.), In Search of Coronary-Prone Behavior, Hillsdale, New Jersey (pp.91-116).Friedman, 1992.

Friedman, M. \& Rosenman, R. (1959) Association of specific overt behavior pattern with blood and cardiovascular findings. Journal of the American Medical Association, 169, 1286-1296.

Friedman, H. (1992) Understanding hostility, coping, and health. In: H.S. Friedman (Ed.) Hostility, Coping, and Health (pp3-9), Washington D.C: American Psychological Association.

Friedman, H., Tucker, J., \& Reisen, S. (1995) Personality dimensions and measures potentially relevant to health: A focus on hostility. Annals of Behavioral Medicine, 17, 245-253.

Friedman, M., Fleischmann, N., \& Price, V.A. (1996) Diagnosis of Type A behavior pattern. In: R. Allan \& S. Scheidt (Eds.) Heart \& Mind. The practice of Cardiac Psychology. APA, Washington, DC (pp 179-196).

Funkenstain, D. King, S., \& Drolette, M. (1954) The direction of anger during a laboratory stressinducing situation. Psychosomatic Medicine, 16, 404-413. 
Gallacher, J.E., Bennett, P. \& Sharp, D.S., (1992) Type A behavior and pressor response in asymptomatic middle-aged men. Psychology and Health, 7, 213-223.

Glass, D.C. (1977) Behavior patterns, stress and coronary disease. Erlbaum, Hillsdale, New Jersey. Golombeck, B., Appels, A., van Breukelen, G., de Vreede, J and Gorgels, A, (1999) Sudden Cardiac Death in an out of hospital population and the risk increasing effect of vital exhaustion.

Gove, W. (1973) Sex, marital status and mortality. American Journal of Sociology. 79, 45-67.

Graff-Low, K. \& Fleisher, C. (1994) Psychosocial correlates f angiograpically defined coronary artery disease in women. Paper presented at the 52cd Annual Meeting of the American Psychosomatic Society, Boston.

Haynes, S., Feinleib, , \& Kannel, W. (1980) The relationship of psychosocial factors to coronary heart disease in the Framingham Heart Study. III Eight year incidence of coronary heart disease. American Journal of Epidemiology, 111, 37-58.

Haynes, S. Feinlieb, M. (1980) Women, work and coronary heart disease: Prospective findings from the Framingham Heart Study. American Journal of Public Health, 70, 133-141.

Haynes, S., Levine, S., Scotch, N., Feinleib, M \& Kannel, W., (1978) The relationship between psychosocial factors to coronary heart disease in the Framingham Study, II. Prevalence of coronary heart disease. American Journal of Epidemiology,

Haynes, S. \& Matthews, K. (1988) Review and methodological critique of recent studies on Type A behavior and cardiovascular disease. Annals of Behavioral Medicine, 10, 47-59.

Hayano, J., Takeushi, S.,Yoshida, S., Jozuka, H., Mishima, N.,\& Fujinami, T. (1989) Type A behavior pattern in Japanese employees: Cross-cultural comparison of major factors in Jenkins Activity Survey (JAS) responses. Journal of Behavioral Medicine, 12, 219-231. Hays, W. (1974). Statistics for the Social Sciences. London: Holt, Rinehart \& Winston.

Hearn, M., Murray, D., \& Luepiker. R. (1989) Hostility, coronary heart disease, and total mortality: A 33-year follow-up study of university students. Journal of Behavioral' Medicine, 12, 105-121.

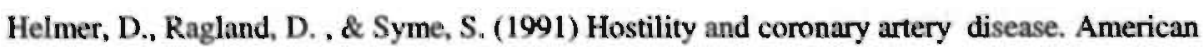
Journal of Epidemiology, 133, i12-122.

Hernandez-Pôzo, R., Muñoz, A., \& Serrano, A. (1996) Análisis de la conducta Tipo "A" en atletas de alto rendimiento. (Análisis of Type A behavior in high standard athletes) ATP Energiay Movimiento, 17, 19-23.

Hernandez-Pozo, R., Hernandez-Pozo, R. \& rodriguez-Ortega, G. (1999) Conducta Tipo "A" en mujeres mexicanas: Un estudio operante. In press. ISBN: 968-36-5616-1.

House, J., Robbins, C., \& Metzner, H. (1982) The associations of social relationships and activities with mortality. Prospective evidence from the Tecumseh Health Study. American Journal of Epidemiology, 116, 567.

House, J., Landis, K. \& Umberson, D. (1988) Social relationships and health. Science, July 29, 241. 
Houston, B. \& Vavak, C. (1991) Cynical hostility: developmental factors, psychosocial correlates, and health behaviors. Health Psychology, 10, 9-17.

Ingram, R. \& Wisnicki, K. (1980). Assessment of positive automatic thinking. Journal of Consulting and Clinical Psychology, 56, 898-902.

Ironson, G., Taylor, C., Boltwood, M., Bartzokis, T., Dennis, C., Chesney, M., Spitzer, S., \& Segall, G. (1992) Effects of anger on left ventricular ejection fraction in coronary disease. American Journal of Cardiology, 70, 281-281.

Jacobs, S. \& Sherwood, J. (1996) The cardiac psychology of women and coronary heart disease. In: R. Allan \& S. Scheidt (Eds.) Heart \& Mind. The practice of Cardiac Psychology.(pp 197-218) APA, Washington, DC,

Jenkins, C. (1988) Epidemiology of cardiovascular diseases. Journal of Consulting and Clinical Psychology, 56, 324-332.

Jenkins, C., Zyzanski, S. \& Rosenman, R. (1978) Coronary-prone behavior: One pattern or several? Psychosomatic Medicine, 40, 25-43.

Julkunen, J., Salonen, R., Kaplan, G., Chesney, M., \& Salonen, J. (1994) Hostility and the progression of carotid atherosclerosis, Psychosomatic Medicine 56, 519.525.

Kaplan, B. (1991) Social health and the forgiving heart. The Type B story. Journal of Behavioral Medicine, 15, 3-14.

Karasek , R. (1990) Lower health risk with increased job control among white-collas workers. Journal of Organizational Behavior, 11, 71-185.

Karlberg, L., Krakau, I., \& Unden, A. (1998) Type A behavior intervention in primary health care reduces hostility and time pressure: A study in Sweden. Social Science and Medicine, 46, 397-402.

Keltinkangas-Järvinen L., Rainkönen, K. \& Hautanen, A. (1996) Type A behavior and

Vital Exhaustion as related to the metabolic hormonal variables of the hypothalamic-pituitaryadrenal axis. Behavioral Medicine, (22), 15-22.

Kempt, S., \& Strongman, K. (1995) Anger theory and management: A historical analysis. In: History of Psychology, Evans, R.B. (Ed.) American Journal of Psychology 108, 397-417.

Klein, S., Stokhof, L. \& Cohen, L. (1994) Vitale uitputting bij patienten met een recent doorgemaakt myocardinfarct. Gedrag \& Gezondheid, 22,198-204.

Kneip, R., Delamater, A., Ismond, T., Milford, C., Salvia, L. \& Schwartz, D. (1993) Self-and spouse ratings of anger and hostility as predictors of coronary heart disease. Health Psychology, 12, 301-307.

Kop, W.; Appels, A., Mendes de Leon, C., de Swart, J. \& Bar, F. (1994). Vital Exhaustion predicts new cardiac events after successful coronary angioplasty. Psychosomatic Medicine, 56, 281-287.

Kop, W, Appels, A., Mendes de Leon C. et al (1996) The relationship between the severity of coronary artery disease and vital exhaustion. Journal of Psychosomatic. Research, 40, 397-405.

Kop, W., Hamulyak, K., Pernot, C., \& Appels, A. (1997) Relationship of blood coagulation and fibrinolysis to vital exhaustion. Psychosomatic Medicine, 60, 352-358. 
Kortnitzer, M., De Backer, G., Dramaix, M. et al., (1980) The Belgian Heart Disease Prevention Project:

Modifications of the coronary risk profile in an industrial population. Circulation, 61, 18-25.

Koskenvuo, M., Kaprio, J., Rose, R., Kesanieme, A., Heikkila, S., \& Langinvainio, K. (1988)

Hostility as a risk factor for mortality and ischemic heart disease in men. Psychosomatic Medicine, 50, 330-340.

Kristensen, M., Kucinskiene, Z., Bergdahl, B., Calkaukas, M. Urmonas, V \& Orth-Gomer, K. (1998) Increased psychosocial strain in Lithuanian versus. Swedish men. Psychosomatic Medicine, 60, 277-282.

Krantz, B., Manuck, S. (1984) Acute psychophysiologic reactivity and risk of cardiovascular disease: A review and methodological critic. Psychologica! Bulletin, 95, 435-464.

Lai, J., \& Linden, W. (1992) Genfer, anger expression, and opportunity for anger release determine cardiovascular reaction and recovery from anger provocation. Psychosomatic Medicine, 54, 297-310.

Langeluddecke, P. Fulcher, G., Jones, M., Tennant, C. (1988) Type A Behavior and coronary atherosclerosis. Journal of Psychosomatic Research, 32, 77-84.

Langan-Fox, J., \& Pool, M. (1995) Occupational stress in Australian business and professional women. Siress Medicine, 11, 113-122.

Lewin, B. (1995) Psychological factors in cardiac rehabilitation. In: D. Jones, \& R. West, (eds.) Cardiac Rehabilitation. BMJ Publishing Group London (pp 83-108)

Linden, W., Chambers, L., Maurice, J., \& Lenz, J. (1993) Sex differences in social support, selfdeception, hostility, and ambulatory cardiovascular activity. Health Psychology, 12, 376380.

Lawler, K.A., \& Schmied, L.A. (1986) Cardiovascualr responsivity, Type A behavior, and parental history of heart disease in young women. Psychophysiology, 23, 28-33.

McDermott, M., (1990) Forms of hostility as risk factors for coronary heart disease. Paper. Oxford, July 2-4.presented at the $4^{\text {th }}$ European Health Psychology Conference.

Manuck, S. (1994) Cardiovascular reactivity in cardiovascular disease: Once more unto the breach. International Journal of Behavioral Medicine, 1,4-31.

Margolis, L., MacLeroy, K.R., Runyan, C.V. \& Kaplan, B.H. (1983) Type A behavior: An ecological approach. Journal of Behavioral Medicine, 6, 217-222.

Maslach, C. (1982) Burnout. The cost of caring. Englewood Cliffs, NJ: Prentice Hall.

Maslach, C. \& Jackson, S. (1986) The Maslach Burnout Inventory. Palo Alto: Consulting Psychologist Press.

Matthews, K. , Glass, D., Rosenman, R., \& Bortner, R. (1977) Competitive drive, patten A, and coronary heart disease: A further analysis of some data from the Western Collaborative Grouo Study. Journal of Chronic Diseases, 30, 489-498.

Matthews, K. (1982) Psychological perspectives on the Type A behavior Pattern. Psychological Bulletin, 91, 293-323. 
Matthwes, K. (1983) Assessement issues in coronary-prone behavior. In: T.M. Dembroski, T.H.

Schmidt \& G. Bluemchen (eds.) Biobehavioral bases of coronary heart disease Base: Karger (pp 62-78).

Matthwes, K. \& Haynes, S. (1986) Type A behavior Pattern and coronary disease: Update and critical evaluation. American Journal of Epidemiology, 123, 923-960

Matthews, K. (1988) Coronary heart disease and Type A behaviors: Update to an alternative to the Booth-Kewley and Friedman (1987) quantitative review. Psychological Bulletin, 104, 373 380.

Matthews, K. (1998). Women's hearts are different from men's. V International Congress of Behavioral Medicine. Copenhagen, 1998

Meesters, C. (1995) Hostility and Vital Exhaustion as risk indicators of myocardial infarction. PhD. Thesis. University of Maastricht. The Netherlands

Meesters, C. \& Appels, A. (1996,a). An Interview to measure Vital Exhaustion. I. Development and comparison with the Maastricht Questionnaire. Psychology and Health, 11, 557-571.

Meesters, C. \& Appels, A. (1996,b). An interview to measure Vital Exhaustion. II. Reliability and validity of the interview and correlations of vital exhaustion with personality characteristics. Psychology and Health, 11, 573-581.

Melamed, S., Harari, G., \& Green, M. (1993) Type A behavior, tension, and ambulatory cardiovascular reactivity in workers exposed to noise stress. Psychosomatic Medicine, 55, 185- 192.

Mendes de Leon, C. (1988) Behavioral and emotional precursors of acute heart disease. PhD thesis. Galveston: University of Texas Medical Branch, USA.

Mendes de Leon, C. \& Meesters, C. (1991) Hostility and anger as risk factors for coronary heart disease. In A. Appels (Ed.) Behavioral Observations in Cardiovascular Research Swets \& Zeitlinger, Amsterdam / Lisse (pp 107-127).

Mendes de Leon, C. \& Meesters "C. (1992) Risk of mortality and coronary heart disease by marital status in middle-aged men in The Netherlands. International Journal of Epidemiology, $2 i(9), 460-466$.

Meneses, R. (1994). Roles múltiples y riesgo cardiovascular en mujeres (Muitiple Roles and cardiovascular risk factors in women). MSc Thesis. Universidad Simón Bolf́var. Caracas.

Micale, D. (1991) Factores de riesgo y protectores en una muestra de mujeres ejecutivas (Psychosocial risk and protective factors in a sample of female managers) MSc Thesis. Universidad Simón Bolívar. Caracas.

Moss, G., Dielman, T., Campanelli, P.. Leech, S., Harian, W., Van Harrison, R., \& Horvath, W. (1986) Demographic correlates of 51 assessments of Type A behavior. Psychosomatic Medicine, 48, 564-574.

Muranaka, M., Lane, J., Suarez, E., Anderson, N., Suzuki, J, \& Williams, R. (1988) Stimulus specific patterns of cardiovascular reactivity in Type A and Type B subjects: Evidence for enhanced vagal' reactivity in Type B. Psychophysiology, 25, 330-338. 
Murza, G. (1990) Prevention in the work-place: An integrative cooperative task. In: L. Schimdt; P. Schwenkmezger, \& S. Maes (eds.) Theoretical aspects of Health Psychology. Hardwood Academic Publishers, New Jersey.

Myrtec, M., \& Greenlee, M. (1984) Psychophysiology of Type A behavior pattern: A critical analysis. Journal of Psychosomatic Research 28, 455-466.

Nelissen-De Vos, Y. (1994) Sleep problems preceding myocardial infarction. PhD Thesis. University of Maastricht. The Netherlands.

ONeill, C., \& Zeichner, A. (1985) Working women: A study of relationships between stress, coping and health. Journal of Psychosomatic Obstetrics and Gynaecology, 4, 105-116.

Orth-Gomer, K. \& Unden, A. (1990) Type A behavior, social support \& coronary risk: Interaction and significance for mortality in cardiac patients. Psychosomatic Medicine, 92, 59-72.

Orth-Gomér, K., Rosengren, A. and Wilhelmsen, L. (1993) Lack of social support and incidence of coronary heart disease in middle-aged Swedish men. Psychosomatic Medicine, 55, 37-43.

Orth-Gomer, K. \& Weiss, S., (1994). Behavioral medicine. Stress Research reports, Swedish National Institute for Psychosocial Factors and Health. Karolinska Institute. 245, 1-7

Patel, C. (1983) A new dimension in the prevention of coronary heart disease. In: T.

Dembroski, Schimidt, T \& Blümchen, G. (eds.) Biobehavioral Basis of Coronary Heart Disease. Karger Biobehavioral Medicine Series 2, Basel.

Perini, C., Nil, R., Bolli, P., Battig, K., \& Buhler, F. (1993) Ischemic ECG changes are found more often in asymptomatic men with coronary prone behavior pattern. Journal of Psychosomatic Research, 37, 355-360.

Phillips, J., Freedman, S., Ivancevich, J., \& Matteson, M. (1991) Type A Behavior, self-appraisal and goal setting. A framework for future research. Journal of Social Behavior \& Personality, 5, 59-76.

Powel, L., Simon, S., Barizokis, T., Patillo, J., \& Thoresen, C. (1991) Emotional arousability predicts sudden cardiac death in post MI men (Abstract). Circulation, 83, 722.

Powel, L. (1996). The Hook: A me3taphor for gaining control of ernotional reactivity. In: R. Allan \& S. Scheidt (Eds.) Heart \& Mind. The practice of Cardiac Psychology. APA, Washington, DC (pp. 313-328).

Ragland, D. \& Brand, R. (1988a) Coronary heart disease mortality in the Western Collaborative Group Study: Follow-up experience of 22 years. American Journal of Epidemiology. 127. $462-475$.

Ragland, D. \& Brand, R. (1988b) Type A behaviour and mortality from coronary heart disease. New England Journal of Medicine, 318, 65-69.

Raikkonen, K., Keskivaara, P., \& Keltinkangas-Jarvinen, L. (1992) Hostility and social support among Type A individuals. Psychology and Health, 7, 289-299.

Ranchor, A. Sanderman, R., Bouma, J., Buunk, B., \& van der Heuvel, W. (1997) An exploration of the relation between hostility and disease. Journal of Behavioral Medicine, 20, 223-239. 
Renault de Moraes, L. Swan, J., \& Cooper, C. (1993) A study of occupational stress among whitecollar workers in Brazil using the occupational stress indicator. Siress Medicine, 9, 9 1-74.

Roberts, W. (1996) Coronary Atherosclerosis__ Description, manifestations, and prevention. In: R. Allan \& S. Scheidt (Eds.) Heart \& Mind. The practice of Cardiac Psychology. APA, Washington, DC (pp. 147-178).

Rosenman, R. \& Friedman, M. (1959) Associations of specific psychological patterns with blood and cardiovascular findings.Joumal of the American Medical Association, 169, 1286-1296.

Rosenman, R., Brand, R., Scholtz, R., \& Friedman, M. (1976) Multivariuate prediction of coronary heart disease during 8.5 year follow-up of the Westerns Collaborative Group Study. American Journal of Cardiology, 37, 903-910.

Rosenman, R., Brand, R., Jenkins, C., Friedman, M., Straus, R. \& Wurm, M. (1975) Coronary Heart Disease in the Western Collaborative Group Study: Final follow-up experience of 8.5 years. Journal of the American Medical Association, 233, 872-877.

Rosenman, R. (1978) The Interview method of assessment of the coronary-prone behavior pattern In T.M. Dembroski, S.W. Weiss, J.L. Shields et al., (eds.) Coronary Prone Behavior New York, NY: Springer Verlag (pp 56-69).

Rosenman, R. \& Chesney, M. (1982) Stress, Type A behavior, and coronary disease. In: L. Goldberg \& S. Brentznitz. (eds.) Handbook of stress. MacMillan, New York.

Rosenman, R. \& Ward, M. (1988) The changing concept of cardiovascular reactivity. Stress Medicine, 4, 241-251.

Roserıman, R. (1990) Cardiovascular reactivity: Physiological and psychological. In: L. Schimdt; P. Schwenkmezger, \& S. Maes Theoretical aspects of Health Psychology. Hardwood Academic Publishers, New Jersey.

Rosenman, R. (1991a) Type A behavior patter: A personal overview. In: Strube, M.J. (Ed.) Type A Behavior Sage Publications, Newbury Park,London. pp. 4-15.

Rosenman, R. (199॥b) Type A behavior and cardiovascular reactivity: Is there a relationship with hypertension?. In: J.R. Gentry \& W. Julius Personality, elevated blood pressure, and essential hypertension, Hemisphere, Texas.

Rosenman R. (1991c) Type A behavior and coronary heart disease. The hostility factor. Stress Medicine, 7, 245-253.

Rozanski, A., Blumenthal, J., Kaplan, J. (1999) Impact of psychosocial factors on the pathogenesis of cardiovascular disease and implications for therapy. Circulation, 99, 2192-2217.

Sartwell, P. (1917) Retrospective studies: A. review for the clinician. Annals of Internal Medicine, 81. 381-386.

Scheidt, S. (1996). A whirlwind tour of cardiology for the mental health professional. In: In: R. Allan \& S. Scheidt (Eds.) Heart \& Mind. The practice of Cardiac Psychology. APA, Washington, DC (pp. 15-62).

Sebregts, HL, Falger, P., Bar, \& Appels, A. (1999) Psychosocial effects of short-term behavioral rehabilitation for coronary patients. In preparation. 
Seeman, T. \& Syme, S. (1987) Social networks and coronary artery disease : A comparison of the structure and function of social relations as predictors of disease. Psychosomatic Medicine, 49, 541-554.

Shapiro, D.„, DePenah, M., Lidagoster, L., Woodring, S., Pierce, D., \& Glassman, A. (1998) Depression after coronary artery bypass graft surgery: Effects on 6-months outcome. Psychosomatics, 39, 224-225.

Shekelle, R., and Ostfeld, A. (1965) Psychometric evaluations of cardiovascular epidemiology , American N Y Academy of Science, 669-675.

Shekelle, R., Gale, M., \& Norusis, M. (1985) For the Research Group. Type A score (Jenkins Activity Survey) and risk of recurrent coronary heart disease in the Aspirin Myocardial Infarction Study. American . Journal of Cardiology, 56, 221-225.

Shekelle, R., Hulley, S., Neaton, J., Billings, J., Borhani, N. Gerace,T., Jacobs, D., Lasser, N., Mittlemark, M., \& Stamler, J. (1985) The MRFIT Behavior Pattern Study: II.Type A behavior and the incidence of coronary heart disease. American Journal of Epidemiology, 122, 559-570.

Scherwitz, L. \& Ornish, D. (1990) The impact of major life-style changes on coronary stenosis, coronary heart disease risk factors and psychological status. Results from the San Francisco Life-Style Heart Trial. Homeostasis in Health \& Disease, 35, 190-197.

Scherwitz, L., McKelvain, R., Laman, C., Patterson, J., Dutton, L., Yusim, S., Lester, J., Draft, I., Rochelle, D., \& Leachman, R. (1983) Type A behavior, self-involvement, and coronary atherosclerosis. Psychosomatic Medicine, 45, 47-57.

Siegman, A., Anderson,R.; Herbst, J.; Boyle, S. \& Wilkinson, J. (1991) Dimensions of anger/hostility and cardiovascular reactivity in provoked and angered men. Journal of Behavioral Medicine, 15, 257-272.

Siegman, A. (1992) Cardiovascular consequences of expressing, experiencing, and repressing anger. Journai of Behavioral Medicine, 16, 539-569.

Siegman, A., Dembroski, T. , \& Crump. D. (1992) Speech rate, loudness, and cardiovascular reactivity. Journal of Behavioral Medicine, 15, 519-532.

Siegman, A., Townsend, S., Blumenthal, R. , Sorkin, J. \& Civelek, A. (1998) Dimensions of anger and CHD in men and women: Self-ratings versus spouse ratings. Journal of Behavioral Medicine, 21, 315-336.

Siegman, A., \& Snow, S. (1997) The outward expression of anger, the inward experience of anger and CVR: The role of vocal expression. Journal of Behavioral Medicine, 20, 29-45.D

Silverman, J. (1977) The human subject in the psychological laboratory. London, Pergamon.

Smith, T: \& Anderson, N. (1986) Models of Personality and Disease: An interactional approach to Type A behavior and cardiovascular risk. Journal of Personality and Social Psychology, 50, $1166-1173$.

Smith, T. , Houston, B. (1987) Hostllity, anger expression, cardiovascular responsivity, and social support. Biological Psychology, 24, 39-48. 
Smith, T. (1989) Interactions, transactions and the Type A behavior pattern: Aditional avenues in the search for coronary-prone behavior. In A.W. Siegman \& T.M. Dembroski /Eds, In Search of Coronary -Prone Behavior, Erlbaum, Hillsdale, New Jersey: Lawrence Erlbaum (pp. 91-116).

Smith, T. (1992) Hostility and Health: Current status of a psychometric hypothesis. Health Psychology, 11, 139-150.

Smith, T., \& Pope, M. (1990) Cynical hostility as a health risk: Current status and future directions. Journal of Social Behavior and Personality, 5, 77-88.

Smith, T. \& Christensen, A. (1992) Cardiovascular reactivity and interpersonal relations:

Psychosomatic processes in social context. Journal of Social and Clinical Psychology, 11, 279-301.

Sorenson, G., Jacobs, D., Pirie, P., Folsom, A., Luepker, R., \& Gilum, R. (1987) Relationships among Type A behavior, employment experiences, and gender: The Minnesota Heart Survey. Journal of Behavioral Medicine, 10, 323-336.

Spicer, J. \& Hong, b. (1991) Interpreting coronary-prone behaviour: relationships among Type A behaviour, hopelessness, anger management and social t. Psychology and Health, 5, 193202.

Spielberger, C.. Johnson, E., Russell, S., Crane, R., Jacobs, G.„\& Worden, T. (1985). The experience and expression of anger. Construction and validation of an anger expressing scale. In: M.A. Chesney \& R.H. Roserıman (Eds.): Anger and hostility in cardiovascular and behavioural disorders. Washington DC: Hemisphere (pp 5-30).

Spielberger, C., Krasner, S. \& Solomon, E. (1988) The experience, expression and control of anger. In M.P. Jannisse (Ed.) Health Psychology: Individual differences and stress. Springer Verlag Publishers. New York.

Spielberger, C. (1992) Type A behavior, anger, hostility, and heart disease. International Journal of Psychology, 27, 6.15.

Sprafka, J., Folson, A., Burke, G., Hahn, L., \& Pirie, P. (1990) Type A. behavior and its associations with cardiovascular disease prevalence in blacks and whites. The Minnesota Heart Survey. Journal of Behavioral Medicine, 13, 1-13.

Stanton-Rich \& Iso-Ahola, (1998) Burnout and leisure. Journal of Applied SocialPsychology, 28, 1931-1950.

Steptoe, A. (1981) Psychological Factors in cardiovascular disorders, Academic Press, London Strube, M. (1987) A self-appraisal model of the Type A behavior pattern. Perspectives in Personality, 2, 201-250.

Suárez, E. \& Williams, R. (1990) The relationship between dimensions of hostility and cardiovascular reactivity as a function of task characteristic. Psychosomatic Medicine, 52, 558-570.

Suls, J. \& Sanders, G. (1989) Why do some behavioral styles place people at risk?. In A.W. Siegman \& T.W. Dembroski (Eds.) In search of coronary-prone behavior: Beyond Type A Lawrence. Erlbaum Associates, Hillsdale, New Jersey (pp. 1-20). 
Swan, G., Carmelli, D., \& Rosenman, R. (1990) Cook-Medley hostility and the Type A Behavior Pattern. Journal of Social Behavior and Personality, 5, 89-106.

Tennant, C., Langeluddecke, P. Fulcher, G., Jones, M.,. (1987) Anger and other psychosocial factors in coronary atherosclerosis. Psychological Medicine, 17, 425-431.

Thomas, S. (1989) Gender differences in anger expression and health implication. Research in Nursing and Health, 12, 389-398.

Thomas, S. P. \& Williams, R.L. (1991) Perceived stress, trait anger, modes of anger expression, and health status of college men and women. Nursing Research, 40, 303-307.

Thoresen, C. and Powell, L. (1992). Type A behavior partern : New perspectives on theory, assessment and intervention. Journal of Consulting and Clinical Psychology, 60, 595-604.

Torosian, T., Lumley, M., Pickard, S. \& Kettere, M. (1997) Silent versus symptomatic myocardial ischemia: The role of psychological and medical factors. Health Psychology, 16, 123-130

Trijsburg, R., Erdman, R., Duivenvoorden, H., Thiel, J., et al (1987) Denial and overcompensation in male patients with MI: An exploratory study of the measurement of defence mechanisms by interpersonal comparison. Psychosomatics, 47, 22-28.

Uchino, B. \& Garvey, T. (1997) The availability of social support reduces cardiovascular reactivity to acute psychological stress. Journal of Behavioral Medicine, 20(1), 15-27.

Ulmer, D. \& Schwartzburd, L. (1996) Theatment of time pathologies. In: Heart \& Mind. The practice of Candiac Psychology. R. Allan \& S. Scheidt (Eds.) APA, Washington, DC. (pp329-362).

Unden, A., Orth-Gomer, K., \& Elofsson, S. (1991) Cardiovascular effects of social support in the work place: twenty-four-hours ECG-monitoring of men and women. Psychosomatic Medicine, 53, 50-60.

Valdes, M. \& Flores, T. (1987) Type A behavior and vulnerability to diseases: A Spanish retrospective study. Siress Medicine, 3, 135-140.

Van Egeren, L. (1991) A "Success Trap" theory of Type A behavior. In Srtube, M.J. (Ed.) Type A Behavior Sage Publications, Newbury Park, London.

Van Diest, R. \& Appels, A. (1991) Vital exhaustion and depression: A conceptual study. Journal of Psychosomatic Research, 35, 355-364.

Waldsein, S., Polefrone, J., Bachen, E., Muldoon, M., Kaplan, J., \& Manuck, S. (1993) Relationshíp of cardiovascular reactivity and anger expression to serum lipid concentrations in healthy young men. Journal of Psychosomatic research, 37, 249-256.

Warwick- Evans, L. (1990) Acute cardiovascular reactivity and haematological responses of Type A and Type B individuals prior to and following examinations. Paper presented at the 1990 Conference of the British Psychophysiology Socielly.

Weidner, G., Boughal, 'T., Connor, S., Pieper, C., \& Mendell, N. (1997) Relationship of job strain to standard coronary risk factors and psychological characteristics in men and women of the Family Heart Study. Health Psychology, 16, 239-247.

Weiss, S. (1992) Behavioral Medicine in the world scene: Toward the year 2000. Annals of Behavioral Medicine, 14, 302-306. 
Williams, R., Haney, T., Lee, K., Kong, W., Blumenthal, J. \& Whalen, R. (1980) Type A behavior, hostility, and coronary atherosclerosis. Psychosomatic Medicine, 42, 539-549.

Williams, R., Lane, J. Kuhn, C., Melosh, W., White, A. \& Schanberg, S. (1982) Type A. behavior and elevated physiological and neuroendocrine responses to cognitive tasks. Science, 218, 483-485.

Williams, R. \& Barefoot, J.C. (1988) Coronary-prone behavior: The emerging role of the hostility complex. In: B.K. Houston \& C.R. Snyder (Eds.). Type A behavior pattern: Research, theory, and intervention. John Wiley \& Sons, Chichester (pp. 189-211).

Williams, R., Barefoot, J., \& Shekelle, R. (1985) The health consequences of hostility. In M.A. Chesney, S.E. Goldman , \& R.H. Rosenman (Eds.) Anger. Hostility and Behaviral Medicine, Hemisphere/MacGraw Hill, New York (pp 173-186).

Williams, R. (1987) Refining the Type hypothesis: he emergence of the hostility complex. American Journal of Cardiology, 60, 27-32.

Williams, R. (1984) Commentary: The diagnosis and quantitative assessment of Type A behaviour: Introduction and description of the Videotape SI. Integrative Psychiatry, 2, 129-131

Williams, R., Suarez, E., Kuhn, C., Zimmerman, E. \& Schanberg, S. (1991) Biobehabioral basis of coronary-prone behavior in middle-aged men. Part I: Evidence for SNS activation in Type As. Psychosomatic Medicine, 53, 517-527.

Willich S., Lewis M., Lowel H., Amtz H., Schubert F., \& Schroder R. (1993) Physical exertion as a trigger of acute myocardial infarction. New England Journai of Medicine, 329(23), 1684-1690.

Wright, L., Abbanato, K., Lancaster, C.., Bourke, M. \& Nielsen, B. (1994) ender-related subcomponent differences in high Type A subjects. Journal of Clinical Psychology, 50(5), 677-680.

Wrzesniewski, K., Forgays, D.G., \& Bonaiuto, P. (1990) Measurament of the Type A behavior pattem in adolescents and young adults: Cross-cultural development of AATAB. Journal of Behavioral Medicine, 13, 111-135.

Yanes, Y. (1991), Factores psicosociales de riesgo cardiovascular en una muestra de policias (Psychosocial risk factor for cardiovascular diseases in a sample of policemen) MSc thesis Simón Bolívar University. Caracas 


\section{SUMMARY}

The study of psychosocial risk factors for myocardial infarction is currently one of the main areas of research within the field of Behavioral Medicine. This is due to the nowadays well-recognized importance of social and psychological concomitants of heart diseases. Several issues have proved controversial or have been considered to deserve further attention. Some of those issues are addressed in this dissertation. Specifically, the main objectives of this thesis are to explore diverse aspects of Type A Behaviour Pattern and Vital Exhaustion, both related in past reports to Coronary Heart Disease.

Five studies were conducted, three on Type A behavior and two on Vital Exhaustion. With the exception of one, all studies were conducted in Caracas, Venezuela. The principal aims of the studies on Type A behavior include exploring the validity of the construct in a Latin American culture, where it has been scarcely investigated, its associations with anger expression styles and social support, Type A-Type B differences in blood pressure and cardiovascular reactivity during the performance of cognitive tasks, and the prevalence of Type A in Venezuelan and British university employees and in Venezuelan managers. The opinions of other informants, spouse and work supervisor, regarding Type A behaviours, anger, and social support of the participants are also of interest. The Vital Exhaustion studies are aimed, first, at the validation of the Spanish versions of the two existing instruments, the Vital Exhaustion Interview and a the Vital exhaustion Questionnaire, second at their pattern of associations with other psychosocial risk factors, and third, at the assessment of the discriminative power of Vital Exhaustion between myocardial infarction patients and controls within the same cultural context. Looking at gender differences in Type A, Vital Exhaustion, and also in anger expression styles, is also of interest throughout the different studies. 
The main results regarding Type A showed that there was a prevalence of $85 \%$ Type As in a sample of high ranking managers in Venezuela, $57 \%$ in university employees in the United Kingdom and $60 \%$ in university employees in Venezuela. Type As did not differ from Type Bs in resting blood pressure but in a measure of blood pressure reactivity at the end of the experimental session on cognitive performance. In another study, spouses and work supervisors did not, or marginally agreed, about participant's behavior indicating that perception of Type A may de situationally determined. Moreover, their level of disagreement was associated with elevated blood pressure in the participants. In both samples of university employees, Venezuelan and British, Type As were more educated than Type Bs but they differed in that in Venezuela there were more Type A women than men whereas the opposite was found in the British sample. Type A was related to anger-out but only in men and to time pressure and impatience but only in women, indicating gender differences in the content of the construct.

To conclude about Type $\mathrm{A}$ and anger expression in a Latin American context, the most salient findings were related to the gender differences and to the possible influence of environmental demands and occupational level on Type A behavior. Conducting prospective studies in this same cultural background seems to be the most appropriate next step in assessing the universality of Type A, anger expression, hostility and lack of social support as risk factors for coronary heart disease.

Vital Exhaustion, on the other hand, proved to be a solid one-factor construct in Venezuela as it had previously been found in The Netherlands. Vital Exhaustion was positively related to all anger expression style measures, to an indicator of negative thinking, and, negatively, to social support. These associations were mainly found when using the; Interview but not so much with the Questionnaire. Type As were found to be more exhausted than Type Bs and women were more exhausted than men. Vital exhaustion discriminated, with a very high odds ratio, myocardial infarction patients from controls. Moreover, the two 
groups differed on anger-in but not in anger-out or anger-control. Vital Exhaustion was related to a measure of negative self-concept in the first study but this association only remained for the myocardial infarction patients in the case-control study. Nevertheless, the discriminative power of Vital Exhaustion was found to be independent of the negative selfconcept.

Thus, the hypothesis, about the universality of Vital Exhaustion as a risk factor for myocardial infarction is strengthened by our results. Furthermore, the boundaries between Vital Exhaustion and other negative mood states such as negative self-concept are somehow more clearly defined. Several issues remain to be explored. These have to do first with the physiological processes leading to the exhaustion feelings and second, with the possible psychosocial antecedents of exhaustion such as life events, job-related stress, and time pathologies.

As from a methodological point of view, we conclude that asking other informants about the participants behavior may be a valuable source of information to assess coronary risk. Besides, the use of interviews rather than questionnaires should be the methodology adopted in order to attain more reliable information. 


\section{SAMENVATTING}

Het onderzoek naar de rol van psychosociale factoren in het ontstaan van het hartinfarct vormt een van de belangrijkste onderzoeksgebieden in de Behavioral Medicine. Dit komt door de heden ten dage algemeen onderkende betekenis van psychologische aspecten van hartziekten. Over verschillende kwesties wordt nog verschillend gedacht. Deze verdienen nader onderzoek. Deze dissertatie gaat op enkele van deze kwesties in. De belangrijkste doelstellingen van deze dissertatie zijn: het verkennen van verschillende aspecten van het Type A Gedrags Patroon (TAGP) en van Vitale Uitputting (VU). Beide factoren zijn in het verleden met Coronaire Hartziekten (CHZ) in verband gebracht.

Er werden vijf studies uitgevoerd, drie over het TAGP en twee over VU. Met uitzondering van een studie werden alle onderzoeken uitgevoerd in Caracas, Venezuela. De belangrijkste doelstellingen van de onderzoeken over het TAGP omvatten een exploratie naar de validiteit van het construct in een Latijns Amerikaanse cultuur, waar het TAGP nauwelijks bestudeerd is, de associatie bij het instituut ondergebracht met verschillende manieren om boosheid uit te drukken en met sociale steun, verschillen tussen A-types en Btypes in bloeddruk en cardiovasculaire reactiviteit tijdens het uitvoeren van cognitieve taken, en de prevalentie van het TAGP bij Venezuelaanse en Britse medewerkers van een universiteit en bij Venezuelaanse managers. Tevens werd aandacht besteed aan het oordeel van andere informanten (de echtgenote en de werkbaas) over het TAGP, de boosheid en de sociale steun van de deelnemers aan het onderzoek.

De studies m.b.t. VU zijn in de eerste plaats gericht op de validatie van de spaanse versie van twee bestaande instrumenten, te weten het Vitale Uitputtings Interview en de Vitale Uitputting Vragenlijst, in de tweede plaats op hun verband met andere psychosociale risicofactoren, en op de derde plaats op het bepalen van het discriminatieve vermogen van VU tussen hartinfarct patiēnten en controles binnen dezelfde culturele context. In verschillende studies werd ook aandacht besteed aan verschillen tussen mannen en vrouwen in TAGP, VU en de wijze waarop boosheid tot uitdrukking wordt gebrache.

De belangrijkste resultaten met betrekking tot het TAGP lieten zien dat de prevalentie van het TAGP bij managers met een hoge positie in Venezuela $85 \%$ bedroeg. Bij medewerkers van een universiteit in het Verenigd Koninkrijk bedroeg dit percentage 
$57 \%$ en bij medewerkers van een universiteit in Venezuela $60 \%$. Type A's verschillen niet van type B's met betrekking tot de bloeddruk in rust.

$\mathrm{Zij}$ verschilden wel in de reactiviteit van de bloeddruk op het einde van het experiment, waarin cognitieve taken werden uitgevoerd.

De stijging van de bloeddruk bleef bij A-types langer aanhouden dan bij B-types. In een andere studie bleek dat echtgenoten en werkbazen niet of slechts marginaal overeenkwamen in hun beoordeling van het gedrag van de deelnemers. Dit geeft aan dat de perceptie van het TAGP situationeel bepaald kan zijn. De mate waarin zij van oordeel verschilden had een positief verband met verhoogde bloeddruk bij de deelnemers. In beide samples van Venezuelaanse en Britse universiteitsmede-werkers bleken A types een hogere opleiding genoten te hebben dan B-types. Het percentage A-types was in Venezuela hoger bij vrouwen dan bij mannen. In het Britse sample werd het omgekeerde gevonden. TAGP was positief verbonden met "anger-out", doch dit verband werd alleen bij mannen aangetroffen. TAGP was ook positief geassocieerd met gevoelens van tijdsdruk en ongeduld, doch dit verband werd alleen bij vrouwen angetroffen. Dit wijst erop dat er geslachts-verschillen bestaan in de inhoud van het construct.

Met betrekking tot het TAGP en het uitdrukken van boosheid in een Latijns Amerikaanse context concluderen wij dat de meest in het oogspringende bevin-dingen betrekking hebben op geslachtsverschillen en op de mogelijke invloed van omgevingseisen en beroepsniveau op het TAGP. Het uitvoeren van prospectief onderzoek tegen deze culturele achtergrond lijkt de meest aangewezen volgende stap om de universaliteit van het TAGP, de wijze waarop boosheid geuit wordt, hostiliteit en gebrek aan sociale steun als risicofactoren voor $\mathrm{CHZ}$ te bepalen.

VU bleek een sterk Eén-factor construct in Venezuela, zoals dat ook eerder in Nederland geconstateerd werd. VU was positief verbonden met alle manieren om boosheid te uiten, met een indicator van negatief denken, en negatief met sociale steun. Deze verbanden werden vooral gevonden wanneer VU gemeten werd m.b.v. het interview en in mindere mate wanneer VU werd gemeten m.b.v. een vragenlijst. Type A's bleken meer uitgeput te zijn dan type B's en vrouwen waren meer uitgeput dan mannen. VU discrimineerde met een zeer hoge odd's ratio tussen hartinfarct patiënten en controles. Bovendien verschilden beide groepen met betrekking tot "anger-in", maar niet met betrekking tot "anger-out" of "anger-control". VU correleerde positief met een index voor negatief zelfbeeld in de eerste studie, doch deze associatie werd in de case-control studie alleen bij de patiënten en niet bij de controles geconstateerd. VU discrimineerde ook tussen patienten en controles wanneer gecontroleerd werd voor negatief zelfbeeld. 
Het onderzoek versterkt de hypothese dat Vitale Uitputting een universele risicofactor voor het krijgen van een hartinfarct is. Bovendien zijn de grenzen tussen VU en andere negatieve gemoeds toestanden zoals negatief zelfbeeld nu enigermate helderder gedefinieerd. Verschillende vragen moeten nog verder onderzocht worden.

Deze hebben vooral betrekking op de physiologische processen die leiden tot gevoelens van uitputting en, op de tweede plaats, op de antecedenten van uitputting zoals life events, stress in de werksituatie, en tijd pathologieěn.

Met betrekking tot de methodologie is onze conclusie dat het bevragen van andere informanten over het gedrag van een deelnemer aan een onderzoek een waardevolle bron kan zijn om informatie te verzamelen om het coronaire risico te bepalen. Bovendien concluderen wij dat aan interviews de voorkeur gegeven moet worden boven vragenlijsten om betrouwbare informatie te verzamelen. 


\section{APPFNDIX 1}

\section{VITAL EXAHUSTION QUESTIONNAIRE (Spanish version)}

\section{CUESTIONARIO SOBRE AGOTAMIENTO VITAL}

A continuaci6n Ud. encontrara una serie de preguntas. Por favor, léalas cuidadosamente y marque para cada una de ellas:

- el signo (+) SI ha experimentado el estado o sentimiento descrito en los últimos 6 MESES.

-el signo (-) si NO ha experimentado ese estado 6 sentimiento EN ESE MISMO PERIODO DE TIEMPO o si no se acuerda hace cuanto tiempo que Ud. se siente así.

- En caso de que Ud. NO ESTE SEGURO de sentir o haber tenido estas sensaciones, favor marque el signo de interrogaci6n (i).

No hay respuestas correctas o incorrectas. El cuestionario es estrictamente confidencial

1. ¿Se siente Ud. cansado con frecuencia?

2. ¿Se ha sentido Ud. últimamente apático o indiferente?

3. ¿Se siente Ud. débil o sin energía?

4. ¿Se molesta Ud. mas fácilmente ahora que antes?

5. ¿Se siente Ud. abatido?

6. ¿Siente Ud. algunas veces que su cuerpo es como una batería descargada?

7. ¿Piensa que no esta produciendo mucho últimamente o que es menos capaz de realizar cosas?.

8. ¿Se despierta a veces sintiéndose exhausto o cansado?

9 ¿Se despierta repetidas veces en la noche?

10. ¿Siente que no puede resolver los problemas diarios de la misma manera 6 como lo hacia en el pasado?

11. ¿Siente que no quiere hacer mas esfuerzos?

12. ¿Se molesta mas ahora por pequeñas cosas que antes?

13. ¿Explota o pierde el control mas fácilmente ahora que antes?

14. ¿Siente que últimamente no tiene suficiente energía para sobrevivir?.

15. ¿ Ha sentido que su apetito sexual ha disminuido? 
16. ¿Se le ha hecho difícill últimamente resolver problemas o actividades mentales que requieren de mucha concentraci6n?

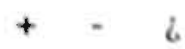

17. ¿Siente cada vez mas dificultad en concentrarse en una actividad por largos periodos de tiempo?

18. ¿Se siente abrumado 6 que tiene que invertir mucho mas esfuerzo?

19. ¿Siente que ha llegado a un callej6n sin salida?

20. ¿A veces llora o tiene ganas de llorar?

21. ¿Se siente derrotado o desilusionado?

22. ¿Se ha sentido desesperanzado recientemente?

23. ¿A veces desea estar muerto?

$+-6$

$+-6$

$+-6$

$+-\zeta_{1}$

$+-6$

$+\quad$ i.

Por favor, antes de devolver el cuestionario, asegúrese de haber RESPONDIDO TODAS LAS PREGUNTAS.

¡MUCHAS GRACIAS!

Nota: La Entrevista de Agotamiento Vital contienen las mismas preguntas. Se modifica la forma de responder, eliminando el signo de interrogación. Ver instrucciones de aplicación. 


\section{APPENDIX 2}

\section{SELF-CONCEPT SUE-SCALE}

Instructions: Thoughts come to our minds without notice. Please, read carefully the following statement and answer if they have appeared in your mind, and if they have how frequently, in the last 6 months. Use the following scale:

$$
\begin{aligned}
& 0=\text { Not at all } \\
& 1=\text { Sometimes } \\
& 2=\text { Frequently } \\
& 3=\text { Almost always }
\end{aligned}
$$

There are no right or wrong answers. The questionnaire is strictly confidential

\begin{tabular}{|l|l|}
\hline English versión & Spanish version \\
\hline 1. My life will improve & 1. Mi vida va a mejorar \\
\hline 2. Everything will work out & 2. Todo va a salir bien \\
\hline 3. It is fun for others to be with me & 3. Es divertido para otros estar conmigo \\
\hline 4. I am proud of my achievements & 4. Estoy orgulloso(a) de mis logros \\
\hline 5. My friends appreciate me & 5. Mis amigos me aprecian \\
\hline 6. My efforts are worthless & 6. Mis esfuerzos no sirven para nada \\
\hline 7. I am a failure & 7. Soy un fracaso \\
\hline 8. No one can understand my problems & 8. Nadie comprende mis problemas \\
\hline 9. I have disapointed others & 9. He decepcionado a otros \\
\hline 10. My future is uncertain & 10. Mi futuro es incierto \\
\hline
\end{tabular}




\section{LIST OF PUBLICATIONS}

Bagés, N. (1990) Estrés y Salud: El papel de los, factores protectores (Stress and Health: the role of protective factors). Comportamiento. 1, 15-28.

Gavidia, L.; Bagés, N., Feldman, L.; Loggiodice, O.; Montilla, G.; Mora, F. \& Passariello, G. (1991) PC based ECG acquisition and analysis system for psychophysiology. Electrocardiography, 15, 825-826.

Feldman, L., Bagés, N. \& Chacón, G. (1991) Factores protectores en la prevención de enfermedades cardiovasculares en la población jóven (Protective factors in the prevention of cardiovascular diseases in the young). Memorias del XXIII Congreso Interamericano de Psicologia. San José de Costa Rica.

Bagés, N., Iribarren C., Canino, E. \& Jaffe, K. (1993) Oxygen consumption and other psychophysiological variables during an active coping task. Acta Cientifica Venezolana, 44, 184-191

Bagés, N., Feldman, L., Vivas, E., Chacón, G., Pérez, M.G. \& Guarino, L. (1994) Prevention studies in the psychophysiology and human behavior group, Simón Bolivar University, Caracas Venezuela. Stress Research Reports. 245, 74-78.

Feldman, L., Bagés, N., \& Chacón, G. (1994) Evaluación Integral y Tratamiento del Temor a Hablar en Público en Estudiantes Universitarios (Assessment and treatment of fear of public speaking in university students) Revista Interamericana de Psicología, 28,

Bagés, N., Feldman, L., \& Chacón,G. (1995) Patrón de Conducta Tipo A y Reactividad Cardiovascular en Gerentes (Type A behavior pattem and cardiovascular reactivity in managers) Salud Püblica de México, 37(1), 47-56.

Feldman, L., Chacón, G., Bagés, N. \& Pérez, M.G. (1996) Stress, gender and occupational level in Venezuelan Employees. In press: In Spielberger, C. \& Sarason, T. (eds.). Stress and Emotion: Anger, Anxiety and Curiosity.

Bagés, N., Falger, P. \& Warwick-Evans, L. Differences between informants about Type A, anger, and social suppont and the relationship with blood Pressure. (1997) Psychology and Health, 12, 453-465.

Bages, N. , Falger, P., Feldman, L., Chacón, G., Pérez, M.G. \&. Guarino, L. (1999) Construct validity of Type A behavior pattern in Venezuela. Submitted, International Journal of Behavioral Medicine. 
Bages, N., Falger, P., Pérez, M.G. \& Appels, A. (2000) Vital Exhaustion Measures and their associations with coronary heart disease risk factors in a sample of Spanishspeakers. Accepted, Psychology \& Health.

Bagés, N., Falger, P., \& Appels, A. (2000) Vital exhaustion as a risk factor of myocardial infarction: A case-control study in Venezuela In press: International Journal of Behovioral Medicine.

Consoli, A., Bagés, N. \& Luchina, C. (2000) La Medicina Comportamental. Qué es y hacia dónde va? (Behavioral medicine: What is it and where is it heading to?) Accepted Revista Interamericana de Psicologia. 


\section{ACKNOWLEDGMENTS}

I am so glad I have so may people to thank!

Doing a doctoral dissertation as an "external student", about a thousand Kilometers away from the University, is not an easy task and it would have an impossible mission had it not been for the support received from so many people from beginning to end.

It is customary to thank the family at the end of the "acknowledgements" section. I cannot but start mentioning them. I have been the luckiest $\mathrm{PhD}$ student for counting with a family as the one I have. Gustavo, Manuel and Tomás are my inspiration and the drive behind nny efforts. Whatever I have achieved with this work, or so many other things in life, I owe it to them. Thanks for being the way you are, for loving me so much, for not complaining when I was away, for making me feel calm during my trips to England and Holland, for encouraging me all the time, for understanding how important this task was for me.

For many reasons, this dissertation has travelled around the world. Living in Venezuela and receiving supervision first form England and then from Holland meant lots of travelling, e-mailing, faxing, and phoning. Given the difficulties in our communication systems, this was not the most pleasant aspect of this work. Thus, I do not thank our national telephone company!

First of all, I must acknowledge the person from whom I learned the basics about Type A and the importance of looking at people's psychosocial aspects in reiation to cardiac health. Back in 1991, Dr. Ray Rosenman, with the passion of the true believer, contaminated me with the need to look at health and illness from a broad and interesting perspective. His suggestions were the spark to initiate this work.

Supervision and data collection started in England, at the University of Southampton, and it was continued in Venezuela with supervision from Holland. Dr. Lawrence Warwick.-Evans, my supervisor during my stay at the UKK, helped me in the first stages of this dissertation when ideas were still vague. I thank him deeply for his respect and confidence. I must also thank the staff and student fellows in the Department of Psychology in Southampton, especially Richard Hastings.

For several reasons the dissertation moved to the University of Maastricht where Professor Ad Appels and Dr. Paul Falger, my promotor and co-promotor respectively, 
guided me trough the forest, helping me to recognize, avoid, jump, and finally ignore! all the obstacles in the path. A summary of what I really learned from them? From Paul I learned how to do research looking at things from as many points of view as possible and that was most enriching. From Ad I learned "to focus" a tool that I just did not know how to use before and that now is part of my everyday vocabulary when confronted with my own students. Without it I would still be running about hypothesis, variables and conclusions. The combination was excellent. The concentrated and substantial meetings that I had with each of them during my short visits to Maastricht, or their short visits to Caracas, proved most effective given their vast knowledge, their enthusiasm, and their interest in what we were doing. I always had this feeling that they would push me to the end. Paul and his family, Pamela, Jeremy and Andrew, were my family and my support when I visited Maastricht and I am most grateful for that. Ad, I admire and respect so much. I greatly enjoyed our meetings that were so stimulating. From him I received support, confidence, patience, friendship, knowledge, and some very good wine and food in between!.

During all the years that this work took, my colleagues/friends at the Simon Bolivar University were at different times my walking sticks, the most attentive ears, and the best shoulders to lean on during the harsh moments of helplessness. From Lya, Eleonora, María Gabriela, Leticia and Grace I received the best that one can get from friends, that is knowing they will be there when needed.

From a financial point of view I thank the "Dirección de Desarrollo Profesoral" at the Universidad Simón Bolivar. I am particularly grateful to Prof. Zunilde de Arenas who, apart from making sure that money was there to travel, exercised the right amount of pressure to make me go on with confidence.

In my visits to England and in his visits to Caracas, Dr. Paul Bennett gave me always-insightful comments and his revisions of some chapters of this thesis were of great help to me not only from the academic point of view but also in helping me with my language difficulties to please the editors!

Computers are the most useful and treacherous tools. As it happens in many cases, this thesis walked on a tight rope several times due to all sorts of software and hardware troubles. When the feeling invaded me that all the work done in so many days or months could be lost I counted with my friends Ana Mejia and David Frost in London, my cousins Albert and Josefina in Barcelona and Daniel Loureiro in Caracas to magically restore files, repair hard disks or advice on fonts or printing problems. Besides, they are all my true friends and all that came with affection and care included. One can ask no more. 
Many people did not believe that I would ever finish this dissertation. I thank them deeply as they increased my inner perception of this enterprise as a challenge.

Inner forces to pursue goals are in great part the product of watching models while growing up. My mother, now dead, and my sister, and also my whole family in law, have always been those models. From them I learned to be persistent, to work hard, and not to quit in difficult times. 


\section{CURRICULUM VITAE}

Nuri Bagés was born on the $17^{\text {th }}$ of September 1950 in Barcelona, Spain. After moving to Venezuela in 1957 she graduated form high school in 1967 in Caracas. After doing two years of Psychology at the Central University of Venezuela, she moved to England and obtained a BSc Psychology degree (Second Class Upper Division Honours) at the University College, University of London in 1976. She worked as a psychologist in regular and special education institutes for a few years and afterwards she followed a Master's degree in Psychology at the Simón Bolivar University graduating in 1984. Since then she has been working at that same University, first as a research assistant and subsequently as half time and full time lecturer teaching undergraduate and postgraduate courses in the fields of psychophysiology stress and health, cognitive-behavioral psychology, and behavioral medicine. Her main fields of interest in research have been psychophysiological reactivity and cognitive processing, fear of public speaking and psychosocial risk and protective factors for cardiovascular diseases. She has been in charge of several posts at the university such as departmental representative in the General Studies Office, Member of the Psychology Department Coordination Councils, Coordinator of Psychology Postgraduate Studies, Head of the Psychophysiology and Human Behavior Section and Principal Member of the Electoral University Committee. In 1995 she founded, and has been the president since, of the Venezuelan Interdisciplinary Group of Behavioral Medicine. In 1997, together with other colleagues, she organized the formation of the Latin American Society of Behavioral Medicine and co-edits the society's Newsletter. She is a member of the Governing Council of the International Society of Behavioral Medicine and co-chair of the Communications Committee of that same scientific group. 

Table 7.I. Summary of the five reported studies

\begin{tabular}{|c|c|c|c|}
\hline Study & Participants & Nieasures & Main Results (Other results in texi) \\
\hline $\begin{array}{l}\text { 1. Type } \mathrm{A} \text { and } \\
\text { cardiovascular reactivity }\end{array}$ & $\begin{array}{l}51 \text { managers ( } 46 \text { men } / 5 \\
\text { women) working in the } \\
\text { national oil company } \\
\text { (Venezuela) }\end{array}$ & $\begin{array}{l}\text {-Type A, Heart Rate, Blood Pressure } \\
\text { Reactivity, Health Habits, Biological } \\
\text { Risk Factors. }\end{array}$ & $\begin{array}{l}-85 \% \text { of the participants were Type A } \\
- \text { Type As and Type Bs did not differ on any of the reactivity measures (HR, } \\
\text { SBP or DBP) during performance of two cognitive tasks but on the measure } \\
\text { taken at the end of the session. } \\
\text {-HR at the beginning of the session was related to level of performance in } \\
\text { tasks } \\
\text {-Type As and Bs differed on personal history of CVD, and hypertension }\end{array}$ \\
\hline $\begin{array}{l}\text { 2. Different informants, } \\
\text { Type } A \text {, anger, social } \\
\text { support and blood } \\
\text { pressure }\end{array}$ & $\begin{array}{l}45 \text { university employees, } \\
\text { partuers and work } \\
\text { supervisors. (England) }\end{array}$ & $\begin{array}{l}\text {-Type A, Anger, Social Support, } \\
\text { Sociodemographic and Health } \\
\text { Indicators, Blood Pressure }\end{array}$ & $\begin{array}{l}\text {-Prevalence of } 60 \% \text { Type As } \\
\text {-Participants and spouses agreed on all measures } \\
\text {-Spouses and work supervisors did not, or marginally, agree about } \\
\text { participants behaviour. } \\
\text {-Type As more educated than Type Bs. } \\
\text { - Level of disagreement between informants on Type A and social support was } \\
\text { associated with elevated blood pressure } \\
\text {-No BP differences ketween Type As and Type Bs. }\end{array}$ \\
\hline $\begin{array}{l}\text { 3. Construct validity of } \\
\text { Type A in Venezuela }\end{array}$ & $\begin{array}{l}231 \text { university employees } \\
\text { (Venezuela) }\end{array}$ & $\begin{array}{l}\text { Type A, Anger Expression, } \\
\text { Sociodemographic and Health } \\
\text { Indicators. }\end{array}$ & $\begin{array}{l}\text { - Prevalence of } 57.4 \% \text { Type As } \\
\text {-More women Type A than men Type A } \\
\text {-Women more impatient/time urgent than men } \\
\text {-Type A S1 related to anger-out, non-verbal hostility and time pressure but } \\
\text { with anger-out only in men and with time pressure only in women. } \\
\text {-Type As more educated than Type Bs. }\end{array}$ \\
\hline $\begin{array}{l}\text { 4. Vital Exhaustion } \\
\text { measures and pantern of } \\
\text { association with other } \\
\text { psychosocial risk factors }\end{array}$ & $\begin{array}{l}230 \text { university employees } \\
\text { (Venezucla) }\end{array}$ & $\begin{array}{l}\text { Vital exhaustion, Type A, Anger } \\
\text { Expression, Social Support, } \\
\text { sociodemographic and Health } \\
\text { Indicators. }\end{array}$ & $\begin{array}{l}\text { - Both, Questionnaire and Interview, are reliable and have one-factor structure } \\
\text {-Type As more exhausted than Type Bs } \\
\text {-VE related to all anger measures. } \\
\text {-VE related to negative thinking } \\
\text {-VE negatively related to social support } \\
\text { - More associations with interview than with questionnaire } \\
\text { - Women more exhausted than men }\end{array}$ \\
\hline $\begin{array}{l}\text { 5. Case-Control study } \\
\text { for Vital Exhaustion }\end{array}$ & $\begin{array}{l}\text { 32. MI patients and } 42 \\
\text { healthy controls } \\
\text { (Venczueia) }\end{array}$ & $\begin{array}{l}\text { Vital Exhaustion, Anger Expression, } \\
\text { Social Support. Positive and } \\
\text { Negative Self-concept, } \\
\text { Sociodemographic and Health } \\
\text { Indicators. }\end{array}$ & $\begin{array}{l}\text {-VE discriminated very well between cases and controls } \\
\text { - Socio-economic status had some influence on association } \\
\text {-Groups differed on anger-in but not on anger-out or anger-control } \\
\text {-VE related to negative thinking only in cases }\end{array}$ \\
\hline
\end{tabular}


